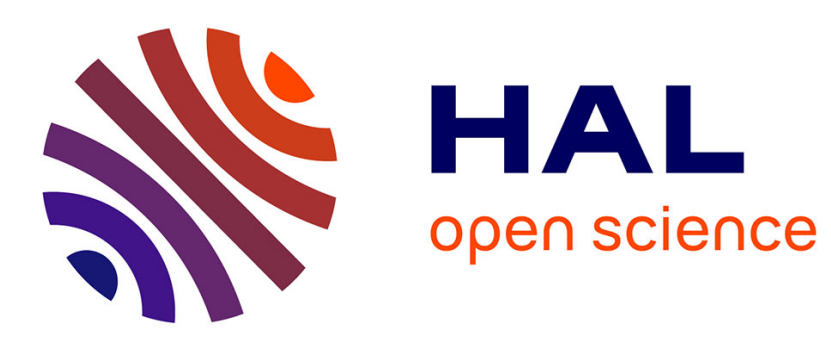

\title{
Some recent Finite Volume schemes to compute Euler equations using real gas EOS
}

\author{
Thierry Gallouët, Jean-Marc Hérard, Nicolas Seguin
}

\section{To cite this version:}

Thierry Gallouët, Jean-Marc Hérard, Nicolas Seguin. Some recent Finite Volume schemes to compute Euler equations using real gas EOS. International Journal for Numerical Methods in Fluids, 2002, 39, pp.1073-1138. 10.1002/fld.346 . hal-01290885

\section{HAL Id: hal-01290885 \\ https://hal.science/hal-01290885}

Submitted on 18 Mar 2016

HAL is a multi-disciplinary open access archive for the deposit and dissemination of scientific research documents, whether they are published or not. The documents may come from teaching and research institutions in France or abroad, or from public or private research centers.
L'archive ouverte pluridisciplinaire HAL, est destinée au dépôt et à la diffusion de documents scientifiques de niveau recherche, publiés ou non, émanant des établissements d'enseignement et de recherche français ou étrangers, des laboratoires publics ou privés. 


\title{
Some recent Finite Volume schemes to compute Euler equations using real gas EOS
}

\author{
Thierry Gallouët, Jean-Marc Hérard, Nicolas Seguin \\ ${ }^{1}$ Laboratoire d'Analyse Topologie et Probabilités - UMR 6632 \\ Centre de Mathématique et Informatique \\ Université de Provence \\ 39 rue Joliot Curie \\ 13453 MARSEILLE CEDEX 13 \\ ${ }^{2}$ Département Mécanique des Fluides et Transferts Thermiques \\ Électricité de France - Recherche et Développement \\ 6 quai Watier \\ 78401 CHATOU CEDEX \\ thierry.gallouet@cmi.univ-mrs.fr \\ herard@cmi.univ-mrs.fr \\ seguin@chi80bk.der.edf.fr
}

\begin{abstract}
This paper deals with the resolution by Finite Volume methods of Euler equations in one space dimension, with real gas state laws (namely perfect gas EOS, Tammann EOS and Van Der Waals EOS). All tests are of unsteady shock tube type, in order to examine a wide class of solutions, involving Sod shock tube, stationary shock wave, simple contact discontinuity, occurence of vacuum by double rarefaction wave, propagation of a 1-rarefaction wave over "vacuum", ... Most of methods computed herein are approximate Godunov solvers : VFRoe, VFFC, VFRoe ncv $(\tau, u, p)$ and PVRS. The energy relaxation method with VFRoe ncv $(\tau, u, p)$ and Rusanov scheme have been investigated too. Qualitative results are presented or commented for all test cases and numerical rates of convergence on some test cases have been measured for first and second order (RungeKutta 2 with MUSCL reconstruction) approximations. Note that rates are measured on solutions involving discontinuities, in order to estimate the loss of accuracy due to these discontinuities.
\end{abstract}


We discuss in this paper the suitability of some Finite Volume schemes to compute Euler equations when dealing with real gas state laws, restricting to the one dimensional framework. Some measured rates of convergence will be presented when focusing on some Riemann problem test cases. This work is based on [43].

Almost all schemes investigated here are approximate Riemann solvers (more exactly approximate Godunov solvers). One may note that comparison with some well known schemes like Godunov scheme or Roe scheme are not provided in this paper ; however, one may refer to [7], [27], [34], [35], [48] for that purpose. Approximate Riemann solvers presented herein may be derived using the general formalism of VFRoe ncv scheme. This only requires defining some suitable variable which is not necessarily the conservative variable, but may be defined on the basis of the solution of the Riemann problem for instance. The first one is obviously VFRoe scheme introduced in [19],[34] and [35], where the candidate is the conservative variable. In the second one, which is known as VFFC scheme, and was introduced in [21], [2] and [31], the privileged variable is the flux variable. The third one, which was introduced some years ago in [5] and with more details in [9], suggests to consider the ${ }^{t}(\tau, u, p)$ variable in the Euler framework. Extensions of the latter scheme to the frame of shallow water equations, or to some non conservative hyperbolic systems arising in the "turbulent" literature are described in [6], [7] and [8]. The fourth one, which applies for the ${ }^{t}(\rho, u, p)$ variable when computing the Euler equations, was introduced by E. F. Toro in [44], [45] and [29], and is known as PVRS (Primitive Variable Riemann Solver). Note that the latter two rely on $(u, p)$ components, which completely determine the solution of the associated Riemann problem, in the sense that assuming no jump on these in the initial conditions results in "ghost" 1-wave and 3-wave. Thus the latter two schemes, which are based on the use of $u$ and $p$ variables, are indeed quite different from the other two, since the former require no knowledge of the one dimensional Riemann problem solution.

Two slightly different schemes are also used for broader comparison. The first one is the Rusanov scheme ([39]), which is known to be rather "diffusive" but anyway enjoys rather pleasant properties, especially when one aims at computing multi dimensional flows on any kind of unstructured mesh. Recall that for Euler type systems, this scheme ensures the positivity of mass and species, provided that the "cell" CFL number is smaller than 1 ([20]). Even more, it requires no entropy correction at sonic points in rarefaction waves, when restricting to "first" order formulation. The last scheme examined is the energy relaxation method proposed by F. Coquel and B. Perthame in [13] (see also [28] and [27] for applications) applied to the frame of VFRoe scheme with ${ }^{t}(\tau, u, p)$ variable. This one again seems appealing both for its simplicity and for its ability to get rid of entropy correction at sonic points in regular fields.

Both "first order" schemes and "second order" schemes (using RK2 time integration and MUSCL reconstruction with minmod limiter on primitive variables) are examined. This includes three distinct EOS, namely :

- perfect gas EOS

- Van der Waals EOS 


\section{- Tammann EOS}

Though complex tabulated EOS are not discussed herein, all above mentionned schemes enable computation of EOS such as those detailed in [37] or [30]. Numerous unsteady tests are performed, involving a wide variety of initial conditions, so that the solution may be either a 1-rarefaction wave with a 3- shock wave, a double shock wave or a double rarefaction wave. We give emphasis on symetric double rarefaction (or shock) waves, since these allow investigation of wall boundary conditions when the standard mirror technique is applied for. The particular experiment of a single isolated contact discontinuity is also described, since the behaviour highly depends on the nature of the state law (see also [42] and [18] on that specific topic). Note also that for almost incompressible fluids, the eigenvalue associated with the LD field is such that the local CFL number varies as $M /(1+M)$, where $M$ stands for the local Mach number, as soon as the overall CFL number is set to 1 . As a result, the accuracy of the prediction of the contact discontinuity is rather poor, which is rather annoying since the vapour quality only varies through this field. Eventually, we note that these test cases include the occurence of vacuum, and the propagation of a shock wave over a (almost) vacuum of gas. The standard stationnary shock is also reported. For completness, we also refer to [32] where Godunov scheme [23] is used to compute Van Der Waals EOS.

Qualitative behaviour of schemes is discussed, and $L^{1}$ error norm is plotted in some cases to provide quantitative comparison. Of course, restricting to smooth solutions, "first order" schemes (respectively "second order" schemes) converges at the order 1 (resp. at the order 2), as exposed for instance in [4] and [33]. Here, solutions investigated here involve some points where the smoothness is only $\mathcal{C}^{0}$ (at the beginning and at the end of rarefaction waves) and even discontinuities (shocks or contact discontinuities). The quantitative study aims at estimating the rate of convergence in such configurations. Several unsteady solutions are presented:

(i)- smooth solutions $\left(\mathcal{C}^{\infty}\right)$,

(ii)- pure contact discontinuities,

(iii)- pure shock waves,

(iv)- rarefaction waves connected with constant states (solutions are not $\mathcal{C}^{1}$ ),

(v)- shock tube test cases which involve several waves.

Both "first" and "second" order schemes are used on these test cases and associated rates of convergence are measured, refining the mesh (with a constant CFL).

\section{Governing equations}

\subsection{Euler equations under conservative form}

Governing Euler equations are written in terms of the mean density $\rho$, the mean pressure $p$, the mean velocity $u$ and the total energy $E$ as follows: 


$$
\frac{\partial W}{\partial t}+\frac{\partial F(W)}{\partial x}=0
$$

setting :

$$
W=\left(\begin{array}{c}
\rho \\
\rho u \\
E
\end{array}\right) \quad, \quad F(W)=\left(\begin{array}{c}
\rho u \\
\rho u^{2}+p \\
u(E+p)
\end{array}\right) \quad \text { and } \quad E=\rho\left(\frac{1}{2} u^{2}+\varepsilon\right)
$$

If $\varepsilon$ denotes the internal energy, then some law is required to close the whole system:

$$
p=p(\rho, \varepsilon)
$$

such that the Jacobian matrix may be diagonalized in $\mathbb{R}$ for $W \in \Omega, \Omega$ the set of admissible states, so that $\hat{\gamma}(p, \rho) p>0, \rho>0$, where :

$$
\left.\rho c^{2}(p, \rho)=\hat{\gamma}(p, \rho) p=\left(\frac{\partial \varepsilon}{\partial p}\right)_{\mid \rho}\right)^{-1}\left(\frac{p}{\rho}-\rho \frac{\partial \varepsilon}{\partial \rho}{ }_{\mid p}\right)
$$

Herein, $c$ stands for the speed of acoustic waves.

The Jacobian matrix $A(W)=\frac{\partial F(W)}{\partial W}$ may be written :

$$
A(W)=\left(\begin{array}{ccc}
0 & 1 & 0 \\
K-u^{2} & u(2-k) & k \\
(K-H) u & H-k u^{2} & u(1+k)
\end{array}\right)
$$

setting:

$$
\begin{aligned}
H & =\frac{E+p}{\rho} \\
k & =\frac{1}{\rho} \frac{\partial p}{\partial \varepsilon} \\
K & =c^{2}+k\left(u^{2}-H\right)
\end{aligned}
$$

Eigenvalues of the Jacobian matrix $A(W)$ read :

$$
\lambda_{1}=u-c, \lambda_{2}=u, \lambda_{3}=u+c
$$

Associated right eigenvectors are :

$$
r_{1}(W)=\left(\begin{array}{c}
1 \\
u-c \\
H-u c
\end{array}\right), r_{2}(W)=\left(\begin{array}{c}
1 \\
u \\
H-\frac{c^{2}}{k}
\end{array}\right), r_{3}(W)=\left(\begin{array}{c}
1 \\
u+c \\
H+u c
\end{array}\right)
$$

Left eigenvectors of $A(W)$ are :

$l_{1}(W)=\frac{1}{2 c^{2}}\left(\begin{array}{c}K+u c \\ -k u-c \\ k\end{array}\right), l_{2}(W)=\frac{k}{c^{2}}\left(\begin{array}{c}H-u^{2} \\ u \\ -1\end{array}\right), l_{3}(W)=\frac{1}{2 c^{2}}\left(\begin{array}{c}K-u c \\ -k u+c \\ k\end{array}\right)$ 
Recall that the 1 -wave and the 3 -wave are Genuinely Non Linear fields and that the 2-wave is Linearly Degenerated. In an alternative way, Euler equations may be written in a non conservative form, when restricting to smooth solutions.

We only provide herein some useful computations of right and left eigenvectors based on non conservative forms of Euler equations.

\subsection{Non conservative form wrt $(\tau, u, p)$}

Let us set $\tau=1 / \rho$. Thus, Euler equations may written in terms of $(\tau, u, p)$ as :

$$
\frac{\partial Y_{1}}{\partial t}+B_{1}\left(Y_{1}\right) \frac{\partial Y_{1}}{\partial x}=0
$$

with

$$
Y_{1}=\left(\begin{array}{l}
\tau \\
u \\
p
\end{array}\right) \quad \text { and } \quad B_{1}\left(Y_{1}\right)=\left(\begin{array}{ccc}
u & -\tau & 0 \\
0 & u & \tau \\
0 & \hat{\gamma} p & u
\end{array}\right)
$$

Obviously, eigenvalues of $B_{1}\left(Y_{1}\right)$ are still :

$$
\lambda_{1}=u-c, \lambda_{2}=u, \lambda_{3}=u+c
$$

Right eigenvectors of matrix $B_{1}\left(Y_{1}\right)$ are :

$$
r_{1}\left(Y_{1}\right)=\left(\begin{array}{c}
\tau \\
c \\
-\hat{\gamma} p
\end{array}\right), r_{2}\left(Y_{1}\right)=\left(\begin{array}{l}
1 \\
0 \\
0
\end{array}\right), r_{3}\left(Y_{1}\right)=\left(\begin{array}{c}
\tau \\
-c \\
-\hat{\gamma} p
\end{array}\right)
$$

Left eigenvectors of $B_{1}\left(Y_{1}\right)$ are :

$$
l_{1}\left(Y_{1}\right)=\frac{1}{2 c^{2}}\left(\begin{array}{c}
0 \\
c \\
-\tau
\end{array}\right), l_{2}\left(Y_{1}\right)=\frac{1}{c^{2}}\left(\begin{array}{c}
1 \\
0 \\
\tau^{2}
\end{array}\right), l_{3}\left(Y_{1}\right)=\frac{1}{2 c^{2}}\left(\begin{array}{c}
0 \\
-c \\
-\tau
\end{array}\right)
$$

\subsection{Non conservative form wrt $(\rho, u, p)$}

In a similar way, we may rewrite Euler equations in terms of $(\rho, u, p)$ :

$$
\frac{\partial Y_{2}}{\partial t}+B_{2}\left(Y_{2}\right) \frac{\partial Y_{2}}{\partial x}=0
$$

with:

$$
Y_{2}=\left(\begin{array}{c}
\rho \\
u \\
p
\end{array}\right) \quad \text { et } \quad B_{2}\left(Y_{2}\right)=\left(\begin{array}{ccc}
u & \rho & 0 \\
0 & u & \frac{1}{\rho} \\
0 & \hat{\gamma} p & u
\end{array}\right)
$$

Right eigenvectors of $B_{2}\left(Y_{2}\right)$ are now :

$$
r_{1}\left(Y_{2}\right)=\left(\begin{array}{c}
1 \\
-\frac{c}{\rho} \\
c^{2}
\end{array}\right), r_{2}\left(Y_{2}\right)=\left(\begin{array}{l}
1 \\
0 \\
0
\end{array}\right), r_{3}\left(Y_{2}\right)=\left(\begin{array}{c}
1 \\
\frac{c}{\rho} \\
c^{2}
\end{array}\right)
$$


Meanwhile, left eigenvectors of matrix $B_{2}\left(Y_{2}\right)$ read :

$$
l_{1}\left(Y_{2}\right)=\frac{1}{2 c^{2}}\left(\begin{array}{c}
0 \\
-\rho c \\
1
\end{array}\right), l_{2}\left(Y_{2}\right)=\left(\begin{array}{c}
1 \\
0 \\
-\frac{1}{c^{2}}
\end{array}\right), l_{3}\left(Y_{2}\right)=\frac{1}{2 c^{2}}\left(\begin{array}{c}
0 \\
\rho c \\
1
\end{array}\right)
$$

\subsection{Non conservative form wrt $F(W)$}

We may rewrite the above mentionned equations in terms of variable $Y=F(W)$.

We multiply on the left by $A(W)$ system (1) :

$$
A(W) \frac{\partial W}{\partial t}+A(W) \frac{\partial F(W)}{\partial x}=0
$$

Since $A(W)$ is the Jacobian matrix of flux $F(W)$, we get :

$$
A(W) \frac{\partial W}{\partial t}=\frac{\partial F(W)}{\partial t}
$$

Hence:

$$
\frac{\partial F(W)}{\partial t}+A(W) \frac{\partial F(W)}{\partial x}=0
$$

The associated matrix still is $A(W)$. Eigenstructure is detailed in 1.1. We now describe the three equations of state used in our computations.

\subsection{Considering various EOS}

\subsubsection{Perfect gas EOS}

The closure law is :

$$
p=(\gamma-1) \rho \varepsilon
$$

with:

$$
\gamma=1,4
$$

\subsubsection{Tammann EOS}

This law is sometimes used to describe the thermodynamics of the liquid phase (see [44]). It may be simply written as :

$$
p=\left(\gamma_{c}-1\right) \rho \varepsilon-\gamma_{c} p_{c}
$$

where:

$$
\gamma_{c}=7,15 \quad p_{c}=3.10^{8}
$$

Actually, using some suitable change of variables enables to retrieve Euler equations with perfect gas state law, assuming $\gamma=\gamma_{c}$. This is an interesting point, since some schemes benefit from nice properties when restricting to perfect gas EOS (see for instance VFRoe with non conservative variable). 


\subsubsection{Van Der Waals EOS}

Van Der Waals EOS is recalled below :

$$
\begin{gathered}
\left(p+\frac{a}{\tau^{2}}\right)(\tau-b)=R T \\
\left.\varepsilon-\varepsilon_{0}=c_{v} T-\frac{a}{\tau_{R}}\right) /(\tau-b) \\
c^{2}=-2 \frac{a}{\tau}+\left(p \tau^{2}+a\right)\left(1+\frac{R}{c_{v}}\right) /(\tau)
\end{gathered}
$$

where:

$$
\begin{aligned}
b & =0,001692 \quad R=461,5 \\
a & =1684,54 \quad c_{v}=1401,88 \\
\varepsilon_{0} & =0
\end{aligned}
$$

This identifies with perfect gas EOS while setting $a=b=0$. This law enables to exhibit some deficiencies of schemes around the contact discontinuity in some cases. We refer to [32] which provides some approximation based on Godunov scheme, when focusing on this particular EOS. Initial conditions in shock-tube experiments are taken in this reference. Comparison with some other test cases can be found in [20], [5] and [9].

\section{Numerical schemes}

\subsection{Framework}

\subsubsection{Finite Volume schemes}

We thus focus herein on some Finite Volume schemes (see for example [22] and [16]). Regular meshes are considered, whose size $\Delta x$ is such that: $\Delta x=x_{i+1 / 2}-$ $x_{i-1 / 2}, i \in \mathbb{Z}$. Let us denote as usual $\Delta t$ the time step, where $\Delta t=t^{n+1}-t^{n}$, $n \in \mathbb{N}$.

We denote $W \in \mathbb{R}^{n}$ the exact solution of the non degenerate hyperbolic system :

$$
\left\{\begin{array}{l}
\frac{\partial W}{\partial t}+\frac{\partial F(W)}{\partial x}=0 \\
W(x, 0)=W_{0}(x)
\end{array}\right.
$$

with $F(W)$ in $\mathbb{R}^{n}$.

Let $W_{i}^{n}$ be the approximate value of $\frac{1}{\Delta x} \int_{x_{i-1 / 2}}^{x_{i+1 / 2}} W\left(x, t^{n}\right) d x$.

Integrating over $\left[x_{i-1 / 2} ; x_{i+1 / 2}\right] \times\left[t^{n} ; t^{n+1}\right]$ provides:

$$
W_{i}^{n+1}=W_{i}^{n}-\frac{\Delta t}{\Delta x}\left(\phi_{i+1 / 2}^{n}-\phi_{i-1 / 2}^{n}\right)
$$

where $\phi_{i+1 / 2}^{n}$ is the numerical flux through the interface $\left\{x_{i+1 / 2}\right\} \times\left[t^{n} ; t^{n+1}\right]$. The time step should comply with some CFL condition in order to guarantee non interaction of numerical waves inside one particular cell, or some other stability requirement. We restrict our presentation to the frame of three point schemes. 
Thus $\phi_{i+1 / 2}^{n}$ only depends on $W_{i}^{n}$ and $W_{i+1}^{n}$, namely $\phi_{i+1 / 2}^{n}=\phi\left(W_{i}^{n}, W_{i+1}^{n}\right)$. Whatever the scheme is, the following consistancy relation should hold :

$$
\phi(V, V)=F(V)
$$

Hence, we present now approximate numerical fluxes $\phi\left(W_{L}, W_{R}\right)$ associated with the 1D Riemann problem :

$$
\left\{\begin{array}{l}
\frac{\partial W}{\partial t}+\frac{\partial F(W)}{\partial x}=0 \\
W(x, 0)= \begin{cases}W_{L} & \text { if } x<0 \\
W_{R} & \text { if } x>0\end{cases}
\end{array}\right.
$$

\subsubsection{VFRoe schemes}

These are approximate Godunov schemes where the approximate value at the interface between two cells is computed as follows. Let us consider some change of variable $Y=Y(W)$ in such a way that $W_{Y}(Y)$ is inversible. The counterpart of above system for regular solutions is :

$$
\frac{\partial Y}{\partial t}+B(Y) \frac{\partial Y}{\partial x}=0
$$

where $B(Y)=\left(W_{, Y}(Y)\right)^{-1} A(W(Y)) W_{, Y}(Y)(A(W)$ stands for the jacobian matrix of flux $F(W))$.

Now, the numerical flux $\phi\left(W_{L}, W_{R}\right)$ is obtained solving the linearized hyperbolic system :

$$
\left\{\begin{array}{l}
\frac{\partial Y}{\partial t}+B(\hat{Y}) \frac{\partial Y}{\partial x}=0 \\
Y(x, 0)= \begin{cases}Y_{L}=Y\left(W_{L}\right) & \text { if } x<0 \\
Y_{R}=Y\left(W_{R}\right) & \text { if } x>0\end{cases}
\end{array}\right.
$$

where $\hat{Y}$ agrees with condition: $\hat{Y}\left(Y_{L}, Y_{L}\right)=Y_{L}$.

Once the exact solution $Y^{*}\left(x / t ; Y_{L}, Y_{R}\right)$ of this approximate problem is obtained, the numerical flux is :

$$
\phi\left(W_{L}, W_{R}\right)=F\left(W\left(Y^{*}\left(0 ; Y_{L}, Y_{R}\right)\right)\right)
$$

Notation In the following we note variables which are computed on the basis of $\bar{Y}$ (obviously, if $\alpha$ is one component of $\bar{Y}$, the relation below holds: $\widetilde{\alpha}=\bar{\alpha}$ ).

Let us set $\widetilde{l_{k}}, \widetilde{\lambda_{k}}$ and $\widetilde{r_{k}}, k=1, \ldots, n$, left eigenvectors, eigenvalues and right eigenvectors of matrix $B(\bar{Y})$ respectively. If $x / t \neq \lambda_{k}, k=1, \ldots, n$, then the solution $Y^{*}\left(x / t ; Y_{L}, Y_{R}\right)$ of linear problem is :

$$
\begin{aligned}
Y^{*}\left(x / t ; Y_{L}, Y_{R}\right) & =Y_{L}+\sum_{x / t>\widetilde{\lambda_{k}}}\left({ }^{t} \tilde{l_{k}} \cdot\left(Y_{R}-Y_{L}\right)\right) \widetilde{r_{k}} \\
& =Y_{R}-\sum_{x / t<\widetilde{\lambda_{k}}}\left({ }^{t} \tilde{l_{k}} \cdot\left(Y_{R}-Y_{L}\right)\right) \widetilde{r_{k}}
\end{aligned}
$$


Let us emphasize that all schemes involved by the VFRoe ncv formalism are approximate Godunov schemes. Note that, contrary to the Godunov scheme, VFRoe ncv schemes cannot be interpreted as projection methods. Hence, no theoritical result exists to ensure a good behaviour of the algorithm when dealing with simulations including states near vacuum (see [15]).

\subsubsection{Entropy correction}

When one numerical eigenvalue associated with the 1 -wave or the 3 -wave vanishes, an entropy correction is needed for above mentionned schemes. If a 1rarefaction wave overlapping the interface is detected, the approximate value at the interface is modified as:

$$
Y^{*}\left(0 ; Y_{L}, Y_{R}\right)=\frac{Y_{L}+Y_{1}}{2}
$$

In a first approach $([5])$, we may assume that overlapping occurs if :

$$
\lambda_{1}\left(W_{L}\right)<0
$$

and if in addition $\widetilde{\lambda_{1}}$ is close to 0 .

An alternative way consists in the proposal of A. Harten and J.M. Hyman in [24], thus checking whether :

$$
\lambda_{1}\left(W_{L}\right)<0<\lambda_{1}\left(W_{R}\right)
$$

This second approach has been applied herein.

\subsection{Basic VFRoe scheme}

This scheme was first proposed in [19], [34] and [35]. It is based on the following choice $Y(W)=W$ and thus $B(\bar{Y})=A(\bar{W})$. Recall that $A(W)$ is the Jacobian matrix of $F(W)$ in the linearized Riemann problem.

\subsection{VFRoe with non conservative variable $(\tau, u, p)$}

We set now $Y(W)={ }^{t}(\tau, u, p)$, where $\tau=1 / \rho$. This scheme was introduced in [5] (see also [9] and [7], [20], [8] for various applications).

With help of left eigenvectors of $B(\bar{Y})$ detailed in 1.2 , and defining $\tilde{\alpha}_{1}$ and $\tilde{\alpha}_{3}$ as :

$$
\begin{aligned}
& \tilde{\alpha}_{1}=\frac{1}{2 \widetilde{c}^{2}}(\tilde{c} \Delta u-\bar{\tau} \Delta p) \\
& \tilde{\alpha}_{3}=-\frac{1}{2 \widetilde{c}^{2}}(\widetilde{c} \Delta u+\bar{\tau} \Delta p)
\end{aligned}
$$

where $\Delta()=.(.)_{R}-(.)_{L}$, intermediate states $Y_{1}$ and $Y_{2}$ read :

$$
Y_{1}=\left(\begin{array}{c}
\tau_{L}+\tilde{\alpha}_{1} \bar{\tau} \\
u_{L}+\tilde{\alpha}_{1} \tilde{c} \\
p_{L}-\tilde{\alpha}_{1} \tilde{\hat{\gamma}} \bar{p}
\end{array}\right) \quad \text { and } \quad Y_{2}=\left(\begin{array}{c}
\tau_{R}-\tilde{\alpha}_{3} \bar{\tau} \\
u_{R}+\tilde{\alpha}_{3} \widetilde{c} \\
p_{R}+\tilde{\alpha}_{3} \tilde{\hat{\gamma}} \bar{p}
\end{array}\right)
$$


Now:

$$
Y_{2}=Y_{1}+\left({ }^{t} \tilde{l_{2}} \cdot\left(Y_{R}-Y_{L}\right)\right) \widetilde{r_{2}}
$$

and last composants of $\widetilde{r_{2}}$ are null, hence $u_{1}=u_{2}$ and $p_{1}=p_{2}$. The approximate solution is thus in agreement with the exact solution of the Riemann problem. Even more, if we assume that initial conditions agree with $\Delta u=0$ and $\Delta p=0$, the following holds $Y_{1}=Y_{L}$ and $Y_{2}=Y_{R}$ (see [9]). This results in the fact that for some particular EOS such as perfect gas EOS and Tammann EOS, cell averages of velocity and pressure are perfectly preserved through the 2-wave, when focusing on single moving contact discontinuity and scheme VFRoe ncv $(\tau, u, p)$ (see [8] and appendix A for a general expression of the EOS).

Another property of this scheme is that single 1-shocks (respectively 3shocks) are preserved in the sense that exact jump conditions and approximate jump conditions arising from linearised system are equivalent, when restricting to perfect gas EOS. In other words, if we set $\sigma$ the speed of the shock wave and $[\alpha]$ the jump of $\alpha$ through this shock wave, then :

$$
-\sigma[W]+[F(W)]=0
$$

and :

$$
-\sigma[Y]+B(\bar{Y})[Y]=0
$$

are the same (see [9] for more details). However, note that this scheme does not fulfill the Roe condition (see [38]).

Eventually, we note that strictly speaking, the value $\tilde{\hat{\gamma}}$ is completely determined for given choice of $Y$. Details concerning the discrete preservation of the positivity of density and pressure intermediate states can be found in [9].

\subsection{VFRoe with non conservative variable $(\rho, u, p)$-PVRS-}

We now set $Y(W)={ }^{t}(\rho, u, p)$. This scheme actually identifies with PVRS (Primitive Variable Riemann Solver) scheme proposed by E.F. Toro, in [45] or [29]. Coefficients $\tilde{\alpha}_{1}$ and $\tilde{\alpha}_{3}$ are now :

$$
\begin{aligned}
& \tilde{\alpha}_{1}=\frac{1}{2 \widetilde{c}^{2}}(-\bar{\rho} \tilde{c} \Delta u+\Delta p) \\
& \tilde{\alpha}_{3}=\frac{1}{2 \widetilde{c}^{2}}(\bar{\rho} \tilde{c} \Delta u+\Delta p)
\end{aligned}
$$

Hence :

$$
Y_{1}=\left(\begin{array}{c}
\rho_{L}+\tilde{\alpha}_{1} \\
u_{L}-\tilde{\alpha}_{1} \tilde{\overline{\underline{c}}} \\
p_{L}+\tilde{\alpha}_{1} \tilde{c}^{2}
\end{array}\right) \quad \text { and } \quad Y_{2}=\left(\begin{array}{c}
\rho_{R}-\tilde{\alpha}_{3} \\
u_{R}-\tilde{\alpha}_{3} \frac{\tilde{\underline{c}}}{\overline{\bar{\rho}}} \\
p_{R}-\tilde{\alpha}_{3} \bar{c}^{2}
\end{array}\right)
$$

Once again, we check that :

$$
Y_{2}=Y_{1}+\left({ }^{t} \tilde{l_{2}} \cdot\left(Y_{R}-Y_{L}\right)\right) \tilde{r_{2}}
$$


so that approximate intermediate states mimic the behaviour of the exact Godunov scheme. Moreover, for perfect gas EOS and Tamman EOS, cell averages of Riemann invariants of the 2-wave are perfectly preserved. Above mentionned remark concerning jump conditions no longer holds, even when restricting to perfect gas EOS.

If we turn now to intermediate states of pressure, we note that PVRS scheme computes:

$$
p_{1}=p_{2}=\bar{p}\left(1-\frac{\hat{\gamma}(\bar{p}, \bar{\rho}) \Delta u}{2 \tilde{c}}\right)
$$

Thus the pressure intermediate states are strictly positive as soon as:

$$
\frac{\Delta u}{\tilde{c}}<\frac{2}{\hat{\gamma}(\bar{p}, \bar{\rho})}
$$

This should be compared with continuous condition for vacuum occurence :

$$
\Delta u<X_{L}+X_{R}
$$

where :

$$
X_{i}=\int_{0}^{\rho_{i}} \frac{c\left(\rho, s_{i}\right)}{\rho} d \rho
$$

where $s_{i}$ denotes the specific entropy. Thus if we restrict to some symetrical double rarefaction wave with perfect gas EOS, we note that the upper bound of $\frac{\Delta u}{\tilde{c}}$ to avoid occurence of vacuum is $\frac{4}{\gamma-1}$ in the "continuous case" and $\frac{2}{\gamma}$ in the "discrete case" for PVRS scheme. Using the standard value $\gamma=1.4$ provides 10 and $\frac{10}{7}$ respectively.

\subsection{VFRoe scheme with flux variable -VFFC-}

This corresponds to the choice : $Y(W)=F(W)$. This scheme VFFC was first introduced in [21] (see also [2] and [31] for further details). The associated 1D Riemann problem is now :

$$
\left\{\begin{array}{l}
\frac{\partial F(W)}{\partial t}+A(\bar{W}) \frac{\partial F(W)}{\partial x}=0 \\
F(W(x, 0))= \begin{cases}F_{L}=F\left(W_{L}\right) & \text { if } x<0 \\
F_{R}=F\left(W_{R}\right) & \text { if } x>0\end{cases}
\end{array}\right.
$$

The interface numerical flux $F^{*}$ is computed with help of eigenstructure of the Jacobian matrix $A(\bar{W})$, as occurs when focusing on basic VFRoe scheme.

\subsection{Rusanov scheme}

Unlike schemes presented above, Rusanov scheme do not solve an approximate Riemann problem at each interface (see [39]). Numerical flux of Rusanov scheme is :

$$
\phi\left(W_{L}, W_{R}\right)=\frac{F\left(W_{L}\right)+F\left(W_{R}\right)}{2}-\frac{1}{2} \lambda_{i+1 / 2}^{M A X}\left(W_{R}-W_{L}\right)
$$


with

$$
\lambda_{i+1 / 2}^{M A X}=\max \left(\left|u_{L}\right|+c_{L},\left|u_{R}\right|+c_{R}\right)
$$

The mean density remains positive as soon as the C.F.L. condition below holds (see $[20]$ for more details) :

$$
\max _{j \in \mathbb{Z}}\left(\left|u_{j}^{n}\right|+c_{j}^{n}\right) \Delta t \leq \Delta x
$$

Note that a similar condition is exhibited in [43] for the Rusanov scheme with a MUSCL reconstruction with minmod slope limiter ([47]).

\subsection{Energy relaxation method applied to VFRoe with non conservative variable $(\tau, u, p)$}

The energy relaxation method was introduced in [13], and used in [27] and [28]. We refer to these references for further details, and only provide herein an algorithmic version to compute the flux $\phi$, resolving the Riemann problem (3) for the Euler equations.

This requires introducing two additional variables $\gamma_{1}$ and $\varepsilon_{2}$ to the conservative ones. Coefficient $\gamma_{1}$ must fulfill the following conditions to reach convergence of the energy relaxation method:

$$
\begin{aligned}
& \gamma_{1}>\sup _{\rho, \varepsilon} \Gamma(\rho, \varepsilon) \quad \text { where } \quad \Gamma(\rho, \varepsilon)=1+\frac{p_{, \varepsilon}}{\rho} \\
& \gamma_{1}>\sup _{\rho, \varepsilon} \gamma(\rho, \varepsilon) \quad \text { where } \quad \gamma(\rho, \varepsilon)=\frac{\rho}{p} p_{, \rho}+\frac{p_{, \varepsilon}}{\rho}
\end{aligned}
$$

where $\varepsilon=E-\frac{1}{2} \frac{(\rho u)^{2}}{\rho^{2}}$ and $p$ is computed using the real $\operatorname{EOS}(2)$.

Internal energy $\varepsilon_{2}$ is defined as follows :

$$
\varepsilon_{2}=\frac{E}{\rho}-\frac{1}{2} \frac{(\rho u)^{2}}{\rho^{2}}-\frac{p}{\left(\gamma_{1}-1\right) \rho}
$$

We may introduce :

$$
W_{1}(\rho, u, p)=\left(\begin{array}{c}
\rho \\
\rho u \\
\frac{1}{2} \rho u^{2}+\frac{p}{\gamma_{1}-1}
\end{array}\right)
$$

and:

$$
F_{1}\left(W_{1}(\rho, u, p)\right)=\left(\begin{array}{c}
\rho u \\
\rho u^{2}+p \\
u\left(\frac{1}{2} \rho u^{2}+\gamma_{1} \frac{p}{\gamma_{1}-1}\right)
\end{array}\right)
$$

The four governing equations are:

$$
\left\{\begin{aligned}
\frac{\partial W_{1}}{\partial t}+\frac{\partial F_{1}\left(W_{1}\right)}{\partial x} & =0 \\
\left(\rho \varepsilon_{2}\right)_{, t}+\left(\rho u \varepsilon_{2}\right)_{, x} & =0
\end{aligned}\right.
$$


with given initial condition :

$$
{ }^{t}\left(\rho, u, p, \varepsilon_{2}\right)(x, 0)= \begin{cases}{ }^{t}\left(\rho_{L}, u_{L}, p_{L}, \varepsilon_{2 L}\right) & \text { if } x<0 \\ { }^{t}\left(\rho_{R}, u_{R}, p_{R}, \varepsilon_{2}\right) & \text { if } x>0\end{cases}
$$

Thanks to these, one may compute the VFRoe-ncv numerical flux pertaining to the latter system which is an hyperbolic system with three distinct eigenvalues which are those of the Euler system. The numerical flux with three components relative to the mass, momentum and energy equations will eventually be defined as follows :

$$
\phi\left(W_{L}, W_{R}\right)=\left(\begin{array}{l}
F_{1,1}^{*} \\
F_{1,2}^{*} \\
F_{1,3}^{*}+\left(\rho u \varepsilon_{2}\right)^{*}
\end{array}\right)
$$

noting $F_{1}^{*}={ }^{t}\left(F_{1,1}^{*}, F_{1,2}^{*}, F_{1,3}^{*}\right)$.

Since we use the VFRoe ncv $(\tau, u, p)$ scheme to solve the four equations system, we get :

$$
\begin{array}{rll}
\left(\rho u \varepsilon_{2}\right)^{*} & =\rho^{*} u^{*} \varepsilon_{2 L} & \text { if } \bar{u}_{L R}>0 \\
& =\rho^{*} u^{*} \varepsilon_{2 R} & \text { if } \bar{u}_{L R}<0
\end{array}
$$

Since $\varepsilon_{2}$ is defined for each Riemann problem resolution, this variable is not continuous in time (a jump occurs at each time step).

\section{Numerical results}

All test cases have been computed for all schemes, but we do not present here all results (see [43], pp.53-451). However, they are all discussed in the following, with some figures to focus on problems in critical configurations. Let us note that VFRoe ncv $(\tau, u, p)$ scheme without entropy correction have been investigated too, in order to emphasize the influence of the energy relaxation method.

Following tests are performed using constant CFL number; however, CFL number slightly increases at the beginning of the computation, from 0,1 to 0,4 in $t \in\left[0 ; T_{M A X} / 4\right]$. Initial conditions refer to different $1 \mathrm{D}$ Riemann problems. The regular mesh contains one hundred nodes.

We present results pertaining to perfect gas, focusing first on qualitative behaviour and then on measurement of $L^{1}$ error norm of four distinct solutions. After, some qualitative results are discussed, related to the Tammann EOS. The configurations of these test cases are similar to perfect gas EOS. At the end, two cases are presented with the Van Der Waals EOS, in order to emphasize some numerical problems through the LD field.

Remark 1 Unless otherwise specified, the average of $\hat{\gamma}$ which is used in all test cases is the following: $0.5\left((\hat{\gamma})_{L}+(\hat{\gamma})_{R}\right)$. The main advantage of this proposal issuing from [5] is that the mean Jacobian matrix has real eigenvalues, provided that initial states have. This is not necessarily true for some non convex EOS when applying for expected value, i.e. : $\tilde{\gamma}=\hat{\gamma}(\bar{Y})$. However, potential drawbacks 
of the former approach will be discussed when necessary. This remark obviously holds for Tammann EOS and Van der Waals EOS, but not for perfect gas state law.

\subsection{Perfect gas EOS - Qualitative behavior}

\section{Case 1.1 Perfect gas EOS - Sod shock tube}

A 1-rarefaction wave travels to the left and a 3 -shock moves to the right end. The contact discontinuity is right going. This case is usually examined but does not provide much information on schemes since discrepancies can hardly be exhibited between all schemes involved herein. However, one can note that "first-order" Rusanov scheme is a little bit more diffusive than others schemes.

\begin{tabular}{|l|l|}
\hline Left State & Right state \\
\hline$\rho_{L}=1$ & $\rho_{R}=0,125$ \\
$u_{L}=0$ & $u_{R}=0$ \\
$p_{L}=10^{5}$ & $p_{R}=10^{4}$ \\
\hline
\end{tabular}

$T_{M A X}=6 \mathrm{~ms}$

\section{Case 1.2 Perfect gas EOS - Supersonic 1-rarefaction wave}

The 1-rarefaction wave contains a sonic point. As a result, for VFRoe ncv schemes, a wrong shock wave may develop at the origin. This is corrected by introducing an entropy correction at sonic point, when focusing on so called first order scheme. This is no longer compulsory when handling MUSCL type reconstruction, which is usually combined with RK2 time integration in order to avoid loss of stability. Note that VFFC scheme without entropy correction also provides a non entropic shock at sonic point, but this appears to be very small when compared with those arising with VFRoe ncv approach with "physical" variables. Moreover, since the energy relaxation method is applied with VFRoe ncv $(\tau, u, p)$ without entropy correction, a small jump can be detected at the sonic point (which vanishes when the mesh is refined). Since first order Rusanov scheme is not based on a linearised Riemann solver, no problem appears at the sonic point. All second order schemes behave in the same way.

\begin{tabular}{|l|l|}
\hline Left State & Right state \\
\hline$\rho_{L}=1$ & $\rho_{R}=0,01$ \\
$u_{L}=0$ & $u_{R}=0$ \\
$p_{L}=10^{5}$ & $p_{R}=10^{3}$ \\
\hline
\end{tabular}

$T_{\text {MAX }}=5 \mathrm{~ms}$ 


\section{Case 1.3 Perfect gas EOS - Double supersonic rarefaction wave}

This case enables to predict the behaviour of the scheme close to wall boundary conditions when applying the mirror technique. Two rarefaction waves are present in the solution when $u_{R}$ is positive. Due to symetrical initial conditions, the contact discontinuity is a ghost wave. We note that in this particular case VFFC scheme no longer provides a convergent solution since it blows up after a few time steps. Though intermediate states of VFRoe ncv scheme are no longer admissible (see [9]) it however provides a convergent solution. As usual, Rusanov scheme is more diffusive than other schemes, but it provides rather good results.

\begin{tabular}{|l|l|}
\hline \multicolumn{1}{|c|}{ Left State } & Right state \\
\hline$\rho_{L}=1$ & $\rho_{R}=1$ \\
$u_{L}=-1200$ & $u_{R}=1200$ \\
$p_{L}=10^{5}$ & $p_{R}=10^{5}$ \\
\hline
\end{tabular}

$$
T_{M A X}=2 m s
$$

\section{Case 1.4 Perfect gas EOS - Double subsonic shock wave}

This case is very similar to the previous one, but two shocks are now travelling to the left and to the right since $u_{R}$ is negative. It corresponds to an inviscid impinging jet on a wall boundary. For supersonic double shock waves with very high initial kinetic energy, small oscillations may occur close to shocks, even when the CFL number is such that waves do not interact. A similar behaviour is observed when computing the case with help of Godunov scheme. Second order schemes create some oscillations, even in a subsonic configuration, except for Rusanov scheme.

\begin{tabular}{|l|l|}
\hline Left State & Right state \\
\hline$\rho_{L}=1$ & $\rho_{R}=1$ \\
$u_{L}=300$ & $u_{R}=-300$ \\
$p_{L}=10^{5}$ & $p_{R}=10^{5}$ \\
\hline
\end{tabular}

$$
T_{M A X}=5 \mathrm{~ms}
$$

\section{Case 1.5 Perfect gas EOS - Stationary 1-shock wave}

This case is usually considered to evaluate the stability of the (expected) stationary 1-shock wave, especially when the scheme does not comply with Roe's condition. In all cases, no instability arises, and all schemes (except for the energy relaxation method which inserts two points in the stationary shock wave profile and Rusanov scheme which smears the wave) actually perfectly preserve the steadyness, whatever the order is. 


\begin{tabular}{|c|l|}
\hline Left State & Right state \\
\hline$\rho_{L}=3 / 4$ & $\rho_{R}=1$ \\
$u_{L}=4 / 3$ & $u_{R}=1$ \\
$p_{L}=2 / 3$ & $p_{R}=1$ \\
\hline
\end{tabular}

$T_{M A X}=100 \mathrm{~s}$

\section{Case 1.6 Perfect gas EOS - Unsteady contact discontinuity}

This case is interesting since it enables to check whether the Riemann invariants of the 2-wave are preserved from a discrete point of view. This essentially depends on the scheme and the EOS (see appendix A). All (first and second order) computed schemes preserve velocity and pressure exactly constant, whereas density jump at the contact dicontinuity is smeared. Note that Rusanov scheme is once again more diffusive than schemes based on a linearised Riemann solver and the energy relaxation method.

\begin{tabular}{|l|l|}
\hline Left State & Right state \\
\hline$\rho_{L}=1$ & $\rho_{R}=0,1$ \\
$u_{L}=100$ & $u_{R}=100$ \\
$p_{L}=10^{5}$ & $p_{R}=10^{5}$ \\
\hline
\end{tabular}

$T_{M A X}=20 m s$

\section{Case 1.7 Perfect gas EOS - Supersonic 1-rarefaction wave propagat-} ing over "vacuum"

This is one difficult test case for all schemes based on approximate Riemann solvers. Moreover, problems may appear due to the fact that computers have to handle round off errors. The analytical solution is close to a pure 1-rarefaction wave over vacuum, since the variations through the LD field and the 3 -shock are not significant. Note that some variables are not defined in vacuum, namely velocity $u$ or specific volume $\tau$. Indeed, for the first order framework, the energy relaxation method applied to VFRoe ncv $(\tau, u, p)$ without entropy correction blows up after few time steps. However, VFRoe ncv $(\tau, u, p)$ scheme with entropy correction provides good results, except in the vacuum area, where velocity profile becomes less accurate on coarse mesh. Other first order schemes (PVRS, VFFC and Rusanov) provide slightly better profiles, even near vacuum. The second order energy relaxation method and second order VFRoe ncv $(\tau, u, p)$ scheme provide good results, though the problem on the velocity profile in the vacuum area remains unchanged. Other second order schemes perform well.

\begin{tabular}{|l|l|}
\hline Left State & Right state \\
\hline$\rho_{L}=1$ & $\rho_{R}=10^{-7}$ \\
$u_{L}=0$ & $u_{R}=0$ \\
$p_{L}=10^{5}$ & $p_{R}=10^{-2}$ \\
\hline
\end{tabular}

$T_{M A X}=1 \mathrm{~ms}$ 


\section{Case 1.8 Perfect gas EOS - Double rarefaction wave with vacuum}

This one too is interesting, since the violation of condition $(\gamma-1)\left(u_{R}-u_{L}\right)<$ $2\left(c_{R}+c_{L}\right)$ results in a vacuum occurence on each side of the origin. Since this test case provides a double supersonic rarefaction wave, VFFC scheme cannot handle these initial conditions, whatever the order. The energy relaxation method applied to VFRoe ncv $(\tau, u, p)$ scheme without entropy correction blows up too, restricting to the first order approximation. However, these two schemes perform well when handling MUSCL reconstruction with RK2 time integration. Moreover, first or second order PVRS, VFRoe and Rusanov schemes preserve density and pressure positivity in this test case and provide good results too (recall that Rusanov scheme maintains positivity of the density under a standard CFL-like condition).

\begin{tabular}{|l|l|}
\hline Left State & Right state \\
\hline$\rho_{L}=1$ & $\rho_{R}=1$ \\
$u_{L}=-3000$ & $u_{R}=3000$ \\
$p_{L}=10^{5}$ & $p_{R}=10^{5}$ \\
\hline
\end{tabular}

$T_{M A X}=1 \mathrm{~ms}$

\subsection{Perfect gas EOS - Quantitative behavior}

We compute here five test cases (unsteady contact discontinuity, double subsonic shock wave, double subsonic rarefaction wave, Sod shock tube, supersonic 1rarefaction wave with 3 -shock wave) with several meshes : 100, 300, 1000, 3000 and 10000 nodes. Numerical rates of convergence of the $L^{1}$ error are measured and presented. Continuous lines refer to first order schemes, whereas dotted lines refer to second order schemes. All results have been obtained using a constant CFL number $\max _{i}\left(\left|u_{i}\right|+c_{i}\right) \Delta t / h=0.5$. In order to provide a detailed analysis of true convergence rate, we distinguish:

(i)- smooth solutions $\left(\mathcal{C}^{\infty}\right)$,

(ii)- pure contact discontinuities,

(iii)- pure shock waves,

(iv)- rarefaction waves connected with constant states (solutions are not $\mathcal{C}^{1}$ ), (v)- shock tube test cases which involve several waves.

When focusing on solutions in $\mathcal{C}^{\infty}$, three points schemes provide order of convergence close to 1 and five points schemes (with a MUSCL reconstruction) provide rates close to 2 . The reader is refered for instance to the work described in references [4] and [33]. In the first reference above, unsteady solutions are simply given by $u(x, t)=\frac{a_{0} x+b_{n}}{t+t_{n}}, u(x, t)-2 \frac{c(x, t)}{\gamma-1}=c_{0}, p(x, t)=(\rho(x, t))^{\gamma}$, which are basic solutions of Euler equations with perfect gas EOS in a one dimensional framework, and indeed correspond to the inner part of a rarefaction wave. This enables to check that expected rate of convergence is achieved focusing either on first order or second order scheme. This classical result no longer holds when the solution involves rarefaction waves (which are only $\mathcal{C}^{0}$ ) or discontinuities such as 
shock waves or contact discontinuities, which is the case in all the next studied solutions. Therefore, one may expect that the speed of convergence (when $\Delta x$ tends to 0 with constant CFL number) slows down. Measure of $L^{1}$ error norm is achieved for unknowns $\rho, u$ and $p$ since the latter two are not expected to vary through the contact discontinuity whatever the initial conditions are.

\section{Case 2.1 Perfect gas EOS - Unsteady contact discontinuity}

We focus here on initial conditions from Case 1.6. Results presented herein have been obtained using VFRoe ncv $(\tau, u, p)$. This test aims at measuring the rate of convergence when the solution involves a pure contact discontinuity. Pertaining to first order schemes, the rate is approximatively $1 / 2$ and the addition of the MUSCL reconstruction with a RK2 method leads to a rate around 2/3 (see results of figure 49).

This preliminary result is important since it enables to explain the differences between Cases 2.2-2.3 (where no jump of density occurs through the contact discontinuity due to symmetry) and Cases $2.4-2.5$ which correspond to classical shock tube experiments.

\section{Case 2.2 Perfect gas EOS - Double subsonic shock wave}

The initial conditions of this test case come from the Case 1.4. The contact discontinuity is a "ghost wave" (no variable jumps through this wave). This explains why the rate of convergence of the first order schemes is slightly higher for density than in the following Cases 2.4-2.5. For all schemes, the rates of convergence for density variable are slightly higher with the first order approximation than with the second order approximation, though the error of the first order schemes is more important. It may be explained by the occurence of tiny oscillations on the intermediate state caused by the second order schemes. Here, all rates are close to 1 , for both first and second order schemes.

\section{Case 2.3 Perfect gas EOS - Double subsonic rarefaction wave}

This concerns Case 1.3, except for the fact that the initial velocity is set to : $u_{L}=-300$. As a result, the double rarefaction wave is subsonic (hence, the VFFC scheme provides meaningful results). Though the solution of this test case is continuous, connections between rarefaction waves and intermediate states are not regular. Thus, rates of convergence equals to 1 for the "first" order schemes and equals to 2 for the "second" order schemes can hardly be expected. Above mentionned remark concerning the density through the contact discontinuity holds. Nonetheless, unlike in previous case, the rate of convergence for $\rho, u$ and $p$ with first order scheme is smaller than 1 . This means that error located at the beginning and at the end of the rarefaction wave affects much the global error, at least on these "coarse" meshes, which is in agreement with description of local $L^{1}$ error in [4]. The rates of convergence of second order schemes are 
with no doubt very close to 1 for all variables. Note that the error associated with the Rusanov scheme is close to the error of other schemes.

We turn now to standard shock tube experiments which involve several waves with true variations of all components. We may expect thus that both $u, p$ will converge with rate 1 when using so called second order scheme, and also that density convergence rate will be close to $2 / 3$.

\section{Case 2.4 Perfect gas EOS - Sod shock tube}

Initial conditions of this test case are the same as the Case 1.1. We recall here that local $L^{1}$ error has been examined in detail in [4], which confirmed that great part of the error was located not only close to the contact discontinuity and the 3 -shock, but also at the beginning and the end of the 1-rarefaction wave. Though Rusanov scheme is less accurate (in terms of error) than other schemes, its rate of convergence is the same. We can note that the rate of convergence of velocity and pressure are the same and higher than the rate of convergence pertaining to density, owing to the contact discontinuity. As expected, the second order schemes converge faster (the slope is close to 1 for velocity and pressure, and a little bit higher than $2 / 3$ for density).

\section{Case 2.5 Perfect gas EOS - Supersonic 1-rarefaction wave}

This refers to the Case 1.2. Though the solution of this test case is composed by the same set of waves, we can measure here the influence of entropy correction for the first order schemes. The rates of convergence are the same as above for all schemes, except for the energy relaxation method. Indeed, the first order approximation provides higher rates of convergence than in the Sod shock tube case. The true rate of convergence in $L^{1}$ norm is hidden by the error associated with the sonic point due to the parametric entropy correction (which is confirmed by experiments with Godunov scheme on shallow water equations).

To conclude, we emphasize that focusing on the Sod tube test, the loss of accuracy is mainly due to the contact discontinuity, since it has been seen that rates of convergence for rarefaction waves or shock waves are greater than rates of convergence provided for a contact discontinuity. Hence, not only the main numerical diffusion is located on contact discontinuities, and poor rates of convergence when dealing with discontinuous solutions are, again, merely due to contact discontinuities.

\subsection{Tammann EOS}

As mentionned in section 1.5.2, one may retrieve by a suitable change of variables the Euler equations with perfect gas EOS from the Euler equations with Tammann EOS. Hence, the vacuum with the Tamman EOS is $\rho=0$ and $p+p_{c}=0$ and the condition for vacuum occurence (5) becomes:

$$
\Delta u<\frac{2}{\gamma_{c}-1}\left(c_{L}+c_{R}\right)
$$


where $c^{2}=\frac{\gamma_{c}\left(p+p_{c}\right)}{\rho}$.

However, this equivalence is only meaningful in the "continuous" framework. Indeed, it no longer holds from a discrete point of view (except for PVRS and VFRoe ncv $(\tau, u, p))$, and numerical results computed with the Tammann EOS are slightly different of previous results, namely with the perfect gas state law.

\section{Case 3.1 Tammann EOS - Subsonic shock tube}

This case is somewhat different from its counterpart with perfect gas EOS, and is based on initial conditions provided in [44]. However, the numerical approximation behaves as its counterpart with perfect gas EOS : all schemes provide good results, and Rusanov scheme is more diffusive than the others.

\begin{tabular}{|c|l|}
\hline Left State & Right state \\
\hline$\rho_{L}=1100$ & $\rho_{R}=1000$ \\
$u_{L}=500$ & $u_{R}=0$ \\
$p_{L}=5.10^{9}$ & $p_{R}=10^{5}$ \\
\hline \multicolumn{2}{|c}{$T_{M A X}=0.6 \mathrm{~ms}$}
\end{tabular}

\section{Case 3.2 Tammann EOS - Sonic rarefaction wave}

Once again, initial conditions are those provided in reference above. Note that the energy relaxation method (with the first order approximation) completely smears the non-entropic shock caused by VFRoe ncv $(\tau, u, p)$. All VFRoe ncv schemes have the same behaviour, and the Rusanov scheme is still more diffusive (first order or second order). Figures provided by first order schemes are presented (figures 1-6).

\begin{tabular}{|c|l|}
\hline Left State & Right state \\
\hline$\rho_{L}=10^{3}$ & $\rho_{R}=10^{3}$ \\
$u_{L}=2000$ & $u_{R}=2000$ \\
$p_{L}=5.10^{8}$ & $p_{R}=10^{6}$ \\
\hline
\end{tabular}

$T_{M A X}=8 m s$

\section{Case 3.3 Tammann EOS - Double subsonic rarefaction wave}

This test case is the counterpart of the Case 1.3. Note that vacuum (ie $\rho=0$, $p+p_{c}=0$ ) can occur within subsonic range, though it does not appear in this test case. Except for first order Rusanov scheme, all schemes compute a glitch (or a spike) at the interface (where the contact discontinuity is located) on the density. 


\begin{tabular}{|l|l|}
\hline Left State & Right state \\
\hline$\rho_{L}=10^{3}$ & $\rho_{R}=10^{3}$ \\
$u_{L}=-300$ & $u_{R}=300$ \\
$p_{L}=10^{9}$ & $p_{R}=10^{9}$ \\
\hline
\end{tabular}

$$
T_{M A X}=0,5 m s
$$

\section{Case 3.4 Tammann EOS - Double subsonic shock wave}

The only difference between this test case and the case presented above is due to the sign of initial velocities. As a result, in spite of rarefaction waves, the solution is composed by two shock waves and a ghost contact discontinuity. The same behaviour on the density can be noted, namely a glitch at the interface (even with the first order Rusanov scheme).

\begin{tabular}{|c|l|}
\hline Left State & Right state \\
\hline$\rho_{L}=10^{3}$ & $\rho_{R}=10^{3}$ \\
$u_{L}=300$ & $u_{R}=-300$ \\
$p_{L}=10^{9}$ & $p_{R}=10^{9}$ \\
\hline
\end{tabular}

$$
T_{M A X}=0,5 \mathrm{~ms}
$$

\section{Case 3.5 Tammann EOS - Stationary 1-shock wave}

A very slight difference may be seen when the average value of $\hat{\gamma}$ is chosen as $0.5\left((\hat{\gamma})_{L}+(\hat{\gamma})_{R}\right)$ instead of $\widetilde{\hat{\gamma}}=\hat{\gamma}(\bar{Y})$ when focusing on VFRoe ncv with variable $(\tau, u, p)$. The shock remains steady only if the the latter choice is considered from a theoretical point of view, which is confirmed by computation. However, other VFRoe ncv schemes provide as accurate results. First or second order Rusanov scheme is very diffusive, and the energy relaxation method introduces three or two points in the shock profile, according to the order of approximation.

\begin{tabular}{|l|l|}
\hline Left State & Right state \\
\hline$\rho_{L}=2.10^{-10}$ & $\rho_{R}=u_{R}^{-1}$ \\
$u_{L}=5.10^{9}$ & $u_{R}=\frac{4 \gamma_{c}}{\gamma_{c}+1} p_{c}+\frac{\gamma_{c}-1}{\gamma_{c}+1} 5.10^{9}$ \\
$p_{L}=p_{C}$ & $p_{R}=p_{L}+u_{L}-u_{R}$ \\
\hline \multicolumn{2}{|c}{$T_{M A X}=10^{-9} \mathrm{~s}$}
\end{tabular}

\section{Case 3.6 Tammann EOS - Unsteady contact discontinuity}

The results provided by all schemes are similar to those provided with the perfect gas EOS (see Case 1.6). Pressure and velocity are exactly preserved (see appendix A), and the jump of density is smeared by all schemes (in particular by the Rusanov scheme). 


\begin{tabular}{|c|l|}
\hline Left State & Right state \\
\hline$\rho_{L}=10^{3}$ & $\rho_{R}=10^{2}$ \\
$u_{L}=10^{3}$ & $u_{R}=10^{3}$ \\
$p_{L}=10^{8}$ & $p_{R}=10^{8}$ \\
\hline
\end{tabular}

$T_{M A X}=2 m s$

\section{Case 3.7 Tammann EOS - Rarefaction wave propagating over vac- uum}

This test computes a 1-rarefaction wave with a sonic point. The 2 -contact discontinuity and the 3 -shock wave are not of significant importance, like in the Case 1.7. We have used in the following last two cases : $\widetilde{\gamma}=\hat{\gamma}(\bar{Y})$. In this case, only VFRoe ncv $(\tau, u, p)$ with RK2-MUSCL integration and (first or second order) Rusanov scheme enable computation (see figures 7-8). Note that the standard choice $0.5\left((\hat{\gamma})_{L}+(\hat{\gamma})_{R}\right)$ results in a blow up of the computation. Initial conditions make all other schemes blow up. These behaviours confirm the discrete difference between perfect gas EOS and Tammann EOS.

\begin{tabular}{|l|l|}
\hline Left State & Right state \\
\hline$\rho_{L}=10^{3}$ & $\rho_{R}=10^{-9}$ \\
$u_{L}=0$ & $u_{R}=0$ \\
$p_{L}=10^{8}$ & $p_{R}+p_{c}=10^{-2}$ \\
\hline
\end{tabular}

$T_{M A X}=0,6 m s$

\section{Case 3.8 Tammann EOS - Vacuum occurence}

This test results like Case 1.8 in a vacuum occurence in the intermediate state. Recall that vacuum can appear though rarefaction waves are not supersonic. As above, VFRoe ncv $(\tau, u, p)$ and Rusanov schemes enable computation. Note that PVRS and VFRoe schemes also perform well in this test (see figures 9-12).

\begin{tabular}{|l|l|}
\hline Left State & Right state \\
\hline$\rho_{L}=10^{3}$ & $\rho_{R}=10^{3}$ \\
$u_{L}=1500$ & $u_{R}=1500$ \\
$p_{L}=10^{9}$ & $p_{R}=10^{9}$ \\
\hline
\end{tabular}

$T_{M A X}=0,6 \mathrm{~ms}$

\subsection{Van Der Waals EOS}

Results of both computations discussed below were achieved using the standard definition for VFRoe ncv $(\tau, u, p)$ and PVRS schemes of the mean of $\hat{\gamma}$ : $0.5\left((\hat{\gamma})_{L}+(\hat{\gamma})_{R}\right)$ instead of : $\widetilde{\gamma}=\hat{\gamma}(\bar{Y})$ when focusing on VFRoe ncv scheme. Differences between results for both choices could hardly be noticed for the following. 


\section{Case 4.1 Van Der Waals EOS - Subsonic 1-rarefaction wave}

Initial conditions below are taken from the paper by Letellier and Forestier [32]. The main advantage of this case is that it clearly exhibits the rather unpleasant behaviour around the contact discontinuity. Though both the exact Godunov scheme and VFRoe scheme with $(\tau, u, p)$ variables predict equal velocity and pressure of intermediate states on each side of the LD field, cell values of both $u$ and $p$ are not in equilibrium (this confirms results of appendix A for the VFRoe schemes with $(\varphi, u, p)$ variable). Obviously this well-known drawback (see [32]) tends to vanish when the mesh size decreases, or when time increases. First order results are provided on figures 13-18.

\begin{tabular}{|l|l|}
\hline Left State & Right state \\
\hline$\rho_{L}=333,33$ & $\rho_{R}=111,11$ \\
$u_{L}=0$ & $u_{R}=0$ \\
$p_{L}=37311358$ & $p_{R}=21770768$ \\
\hline
\end{tabular}

$T_{M A X}=5 \mathrm{~ms}$

\section{Case 4.2 Van Der Waals EOS - Moving contact discontinuity}

Initial conditions are similar to those given in Case 1.6. Note that the Riemann invariants $u$ and $p$ are not very well preserved around the contact discontinuity when using coarse meshes, and "first" order scheme (see appendix A for more details on VFRoe ncv schemes with $(\varphi, u, p)$ variable). The "second' order version of the scheme performs much better. Unlike sometimes heard, we emphasize that the approximation is still convergent. Small oscillations apart from the LD scheme which were reported in [32] do not arise when using approximate Godunov schemes, which is still unexplained and rather amazing. Due to the very small rate of convergence measured in the LD field (smaller than 2/3), it is clear that this slows down the whole rate of convergence on both velocity and pressure variable, compared with what happens when focusing on perfect gas EOS. Hence, none among schemes presented here are able to preserve velocity and pressure constant on a given mesh (see figures 19-24 for results performed by first order schemes).

\begin{tabular}{|c|l|}
\hline Left State & Right state \\
\hline$\rho_{L}=1$ & $\rho_{R}=10$ \\
$u_{L}=100$ & $u_{R}=100$ \\
$p_{L}=10^{5}$ & $p_{R}=10^{5}$ \\
\hline
\end{tabular}

$T_{M A X}=6 \mathrm{~ms}$ 
- VFRoe ncv (Tau,u,p) 1-1 without entropy correction

+ VFRoe ncv $(T a u, u, p) 1-1+$ Relaxation

(a)

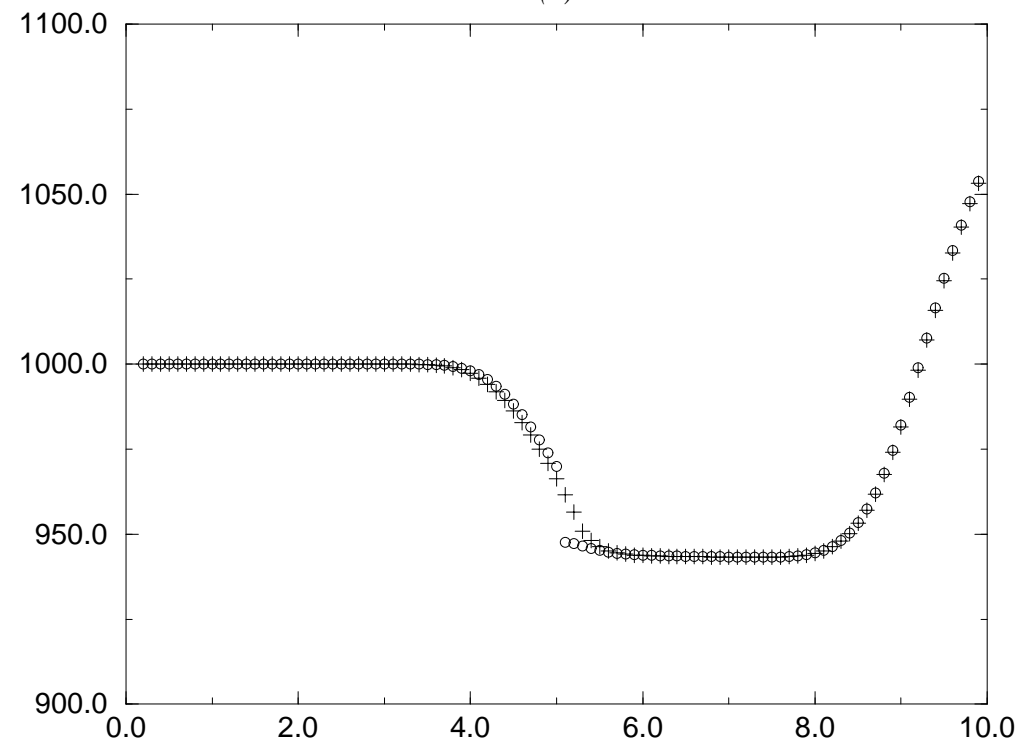

(b)

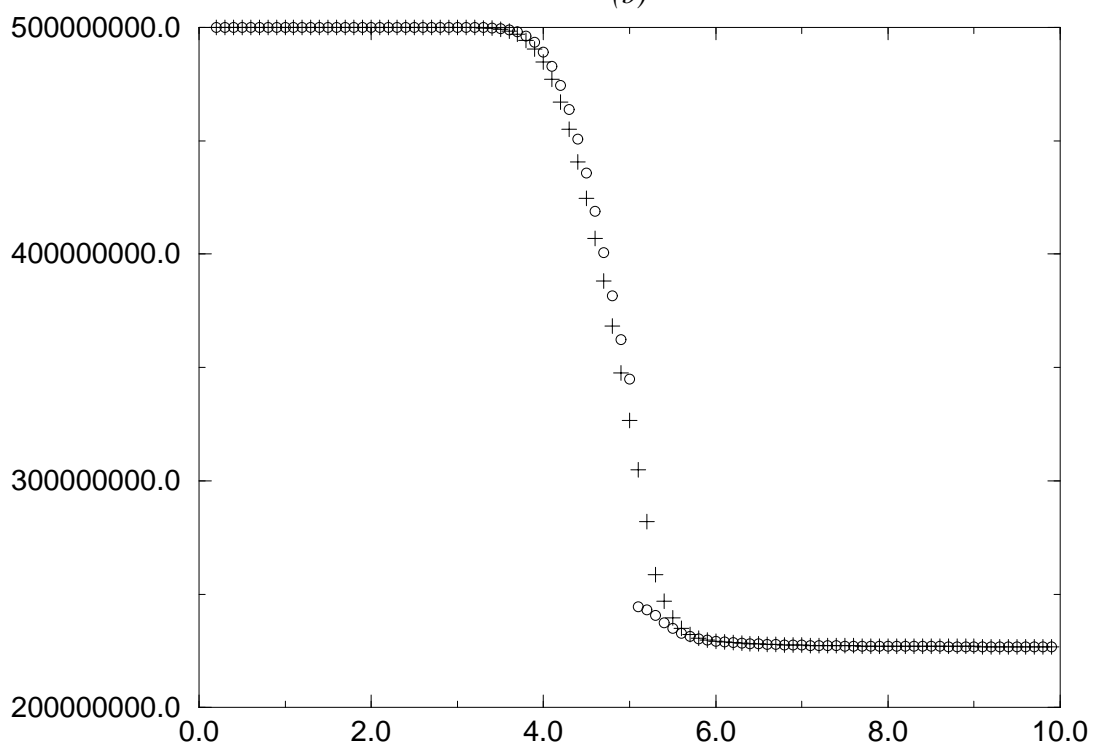

Figure 1: Case 3.2 : density (a) $-p+p_{c}$ (b) 
- VFRoe ncv (Tau,u,p) 1-1 without entropy correction

+ VFRoe ncv $($ Tau,u,p) 1-1 + Relaxation

(a)

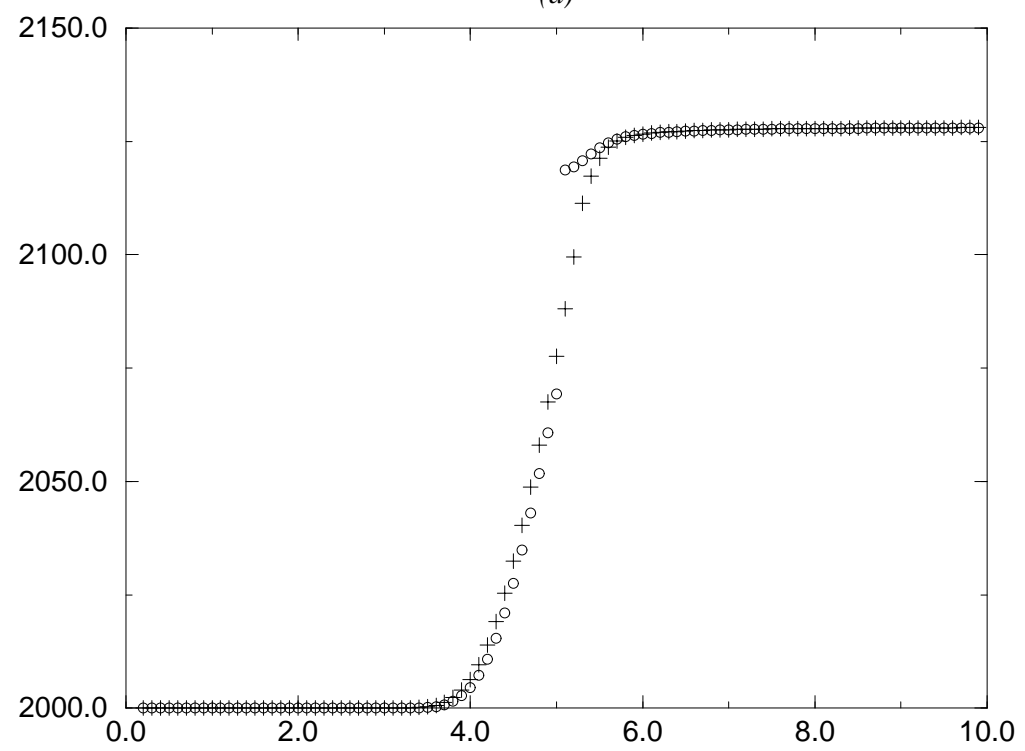

(b)

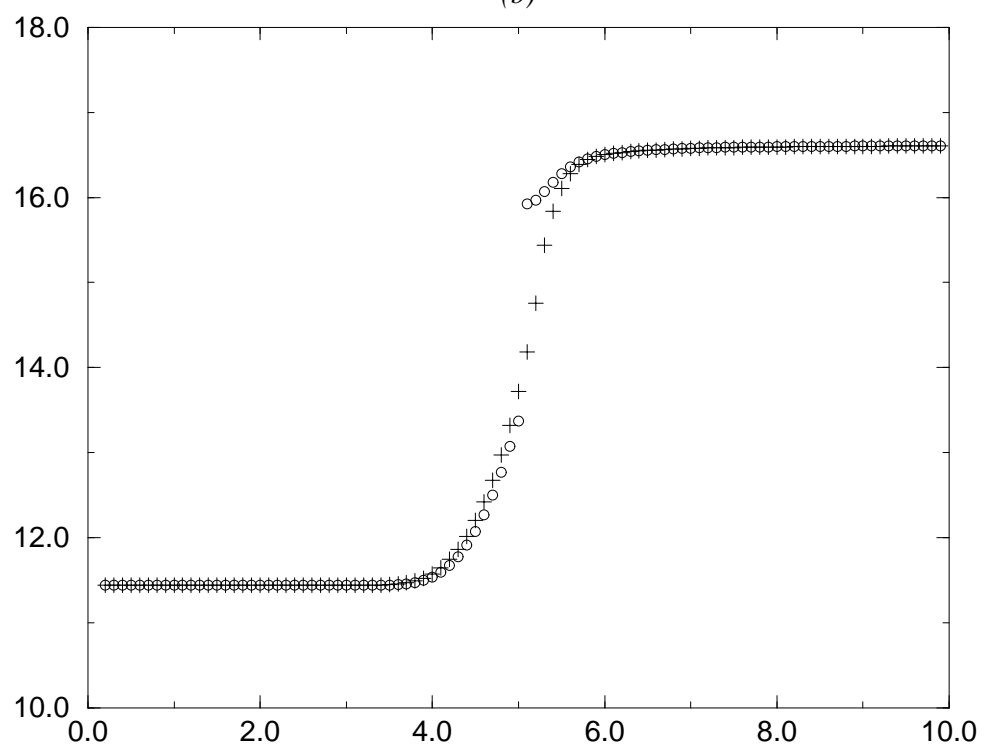

Figure 2: Case 3.2 : velocity (a) $-\hat{\gamma}(p, \rho)$ (b) 
- VFRoe ncv (Tau,u,p) 1-1

+ VFRoe ncv (Rho,u,p) 1-1

$\times$ VFFC 1-1

(a)

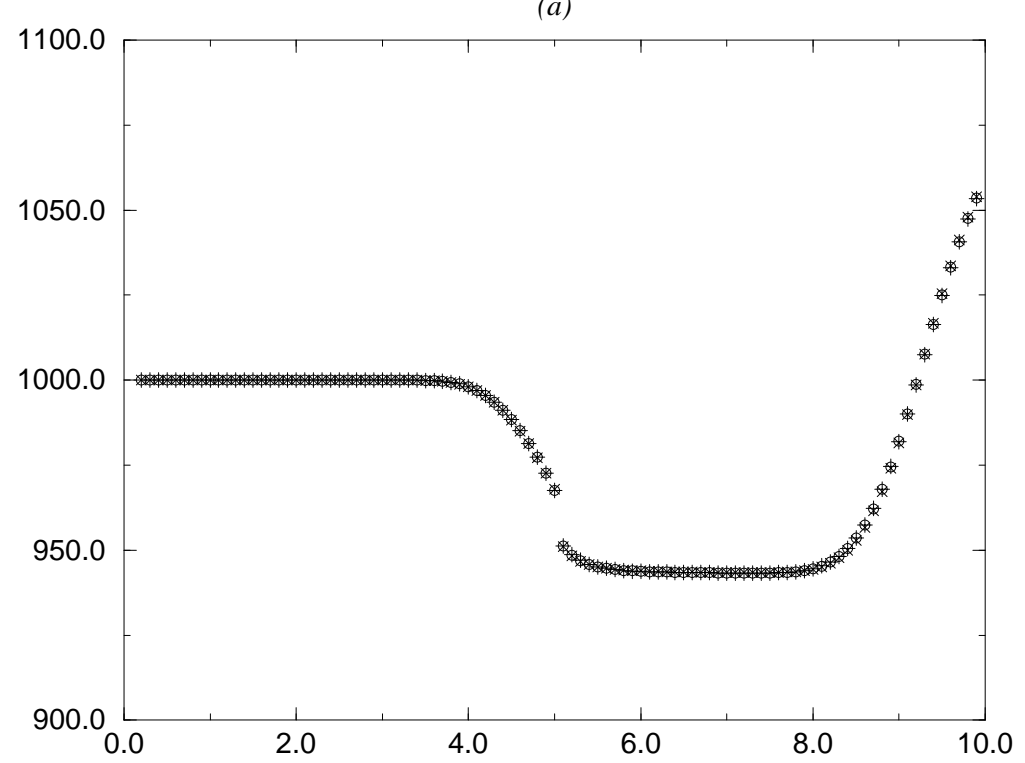

(b)

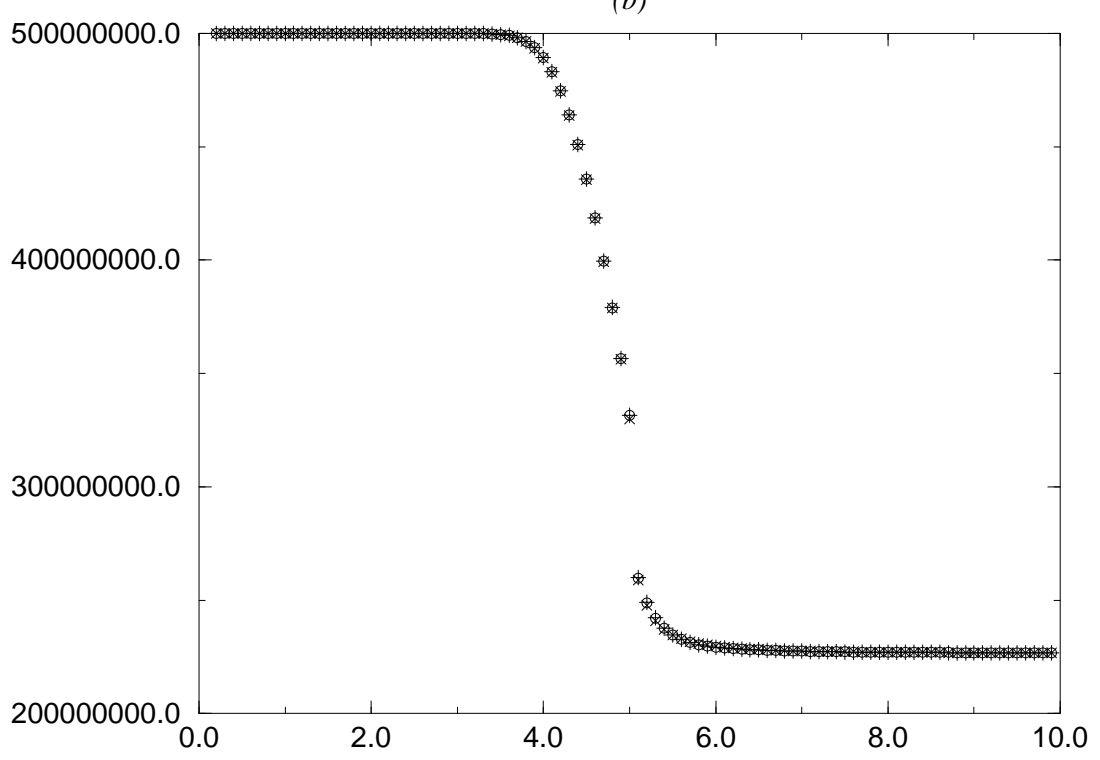

Figure 3: Case 3.2 : density (a) $-p+p_{c}$ (b) 
+ VFRoe ncv (Rho,u,p) 1-1

$\times$ VFFC 1-1

(a)

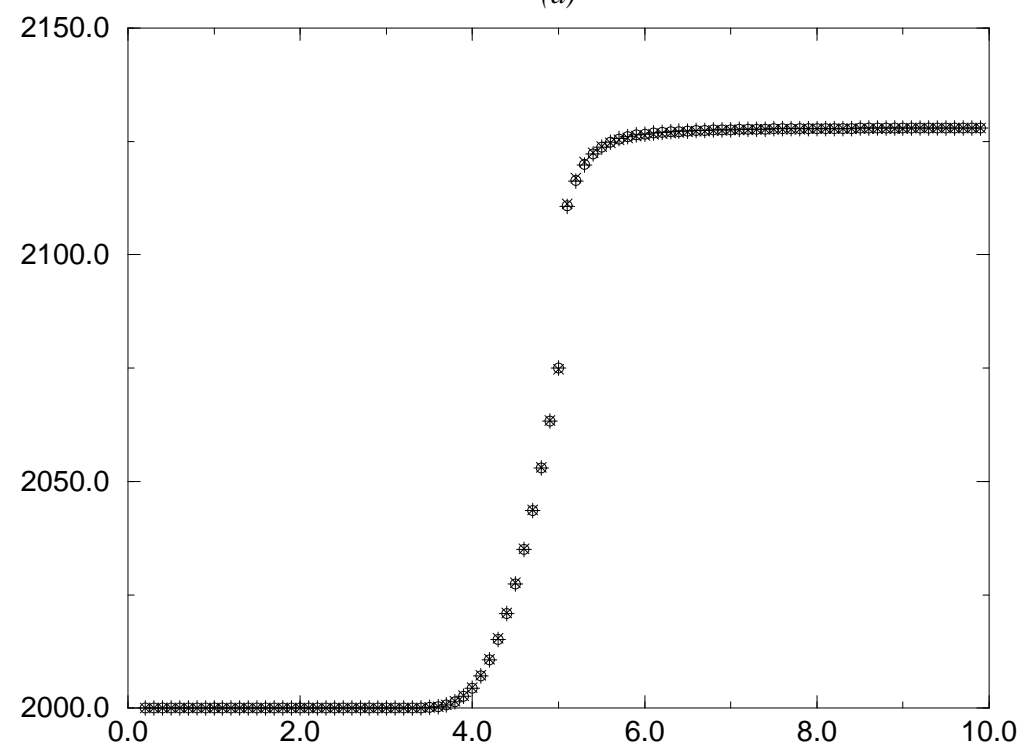

(b)

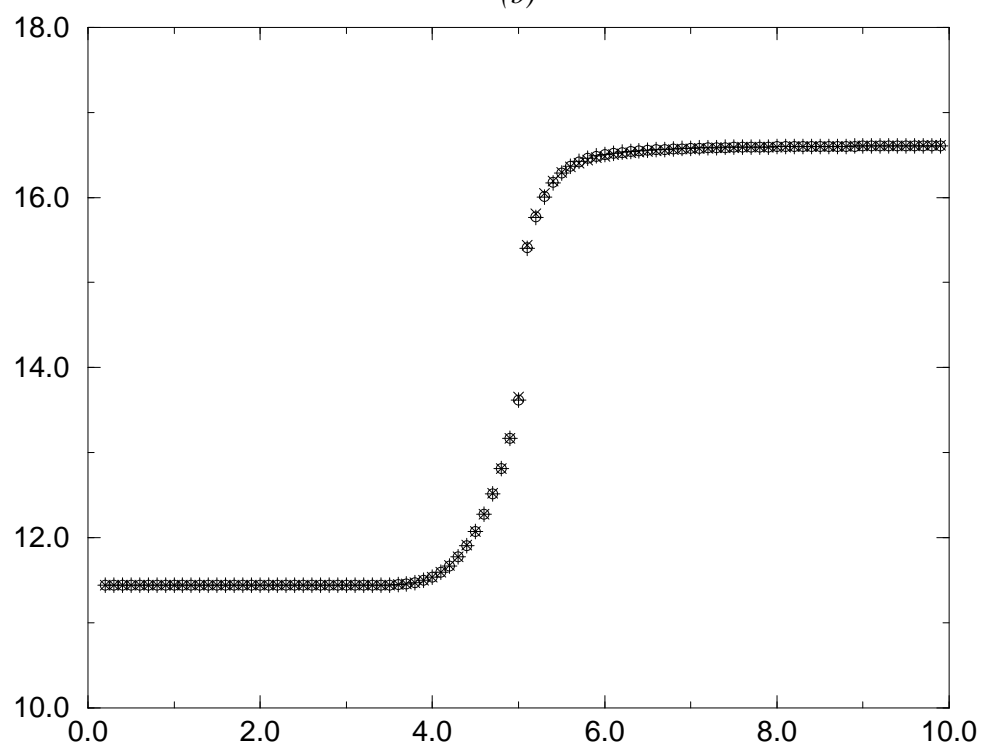

Figure 4: Case 3.2 : velocity (a) $-\hat{\gamma}(p, \rho)$ (b) 
+ VFRoe 1-1

$\times$ Rusanov 1-1

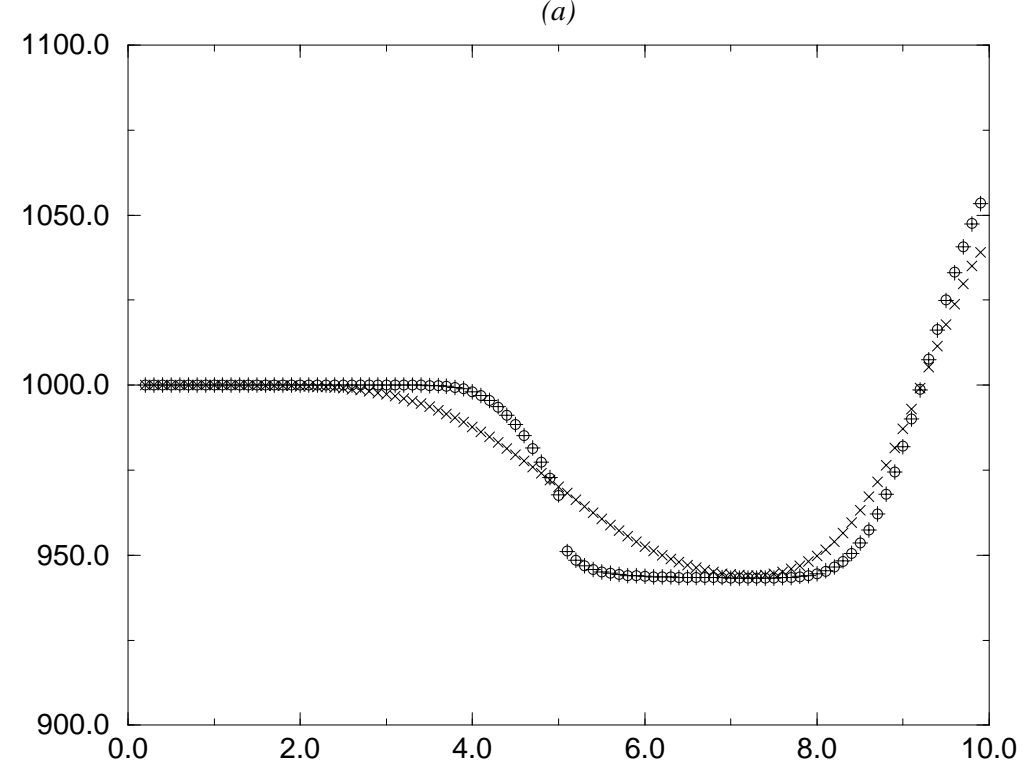

(b)

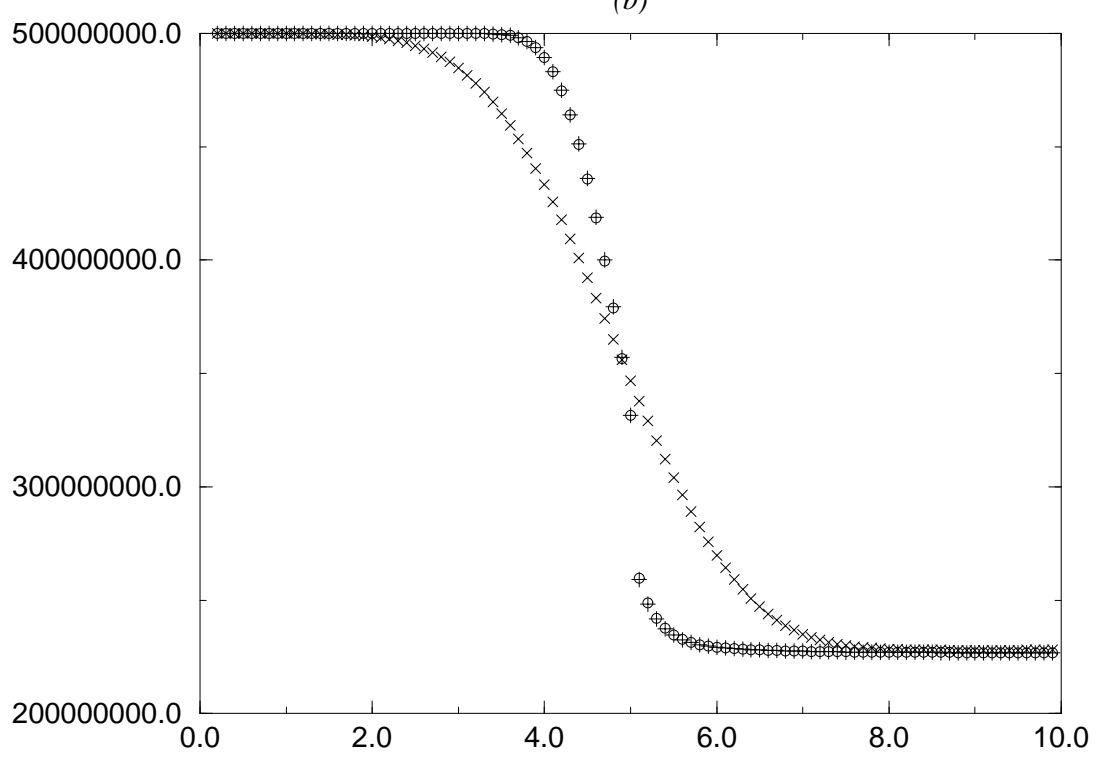

Figure 5: Case 3.2 : density (a) $-p+p_{c}$ (b) 
+ VFRoe 1-1

× Rusanov 1-1

(a)

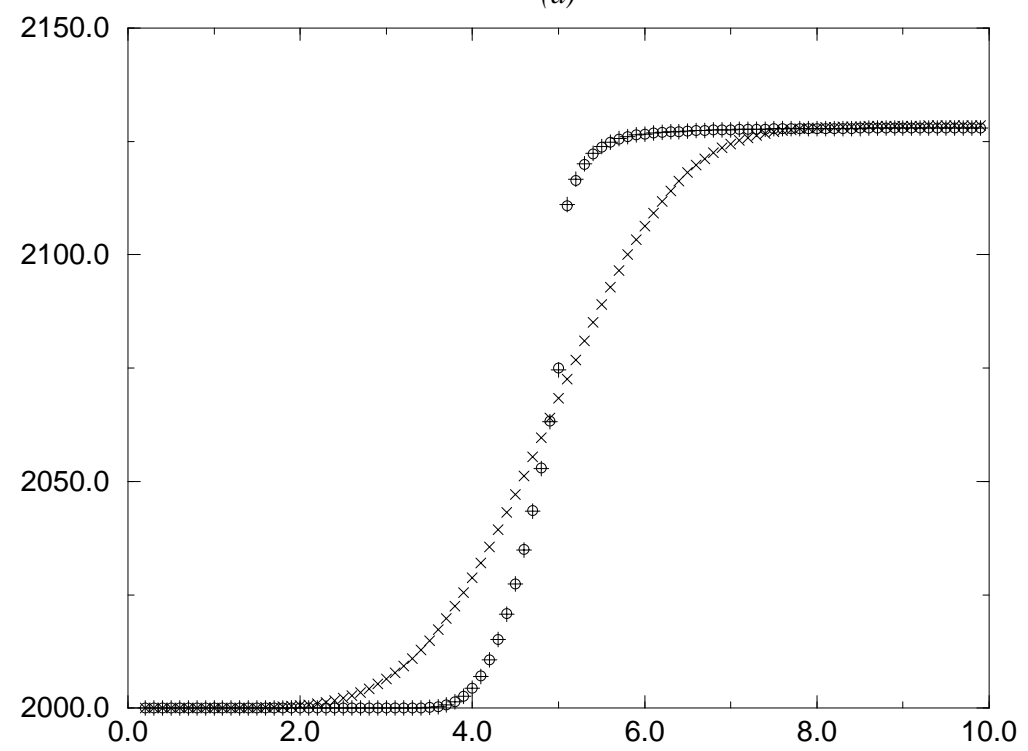

(b)

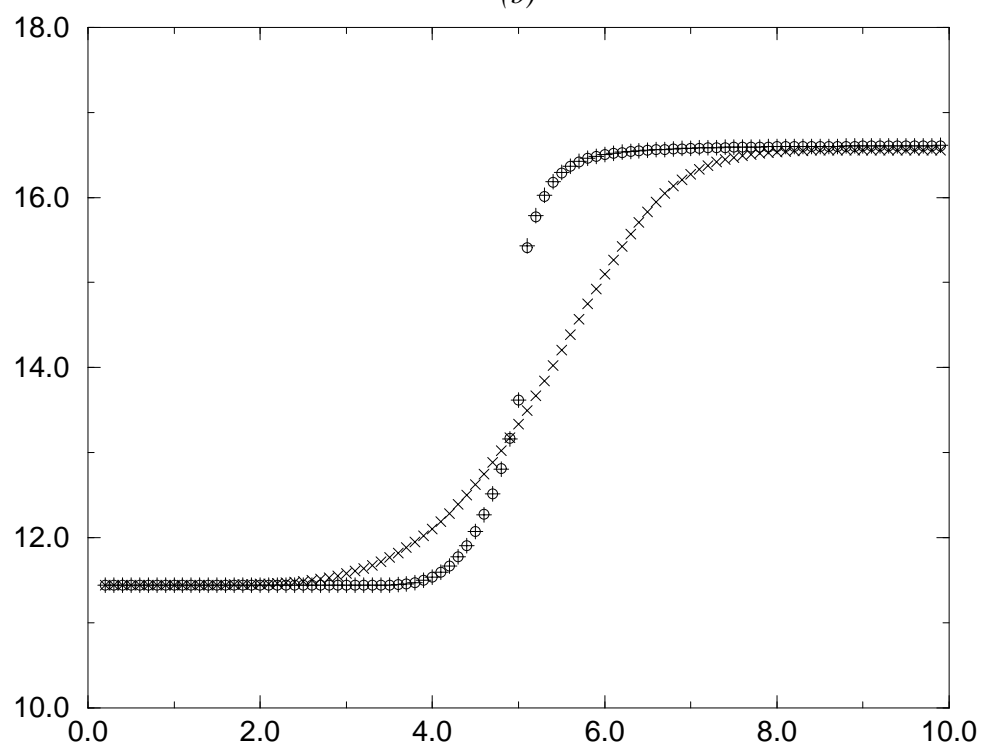

Figure 6: Case 3.2 : velocity (a) $-\hat{\gamma}(p, \rho)$ (b) 
$\times$ Rusanov 2-2

+ VFRoe ncv (Tau,u,p) 2-2
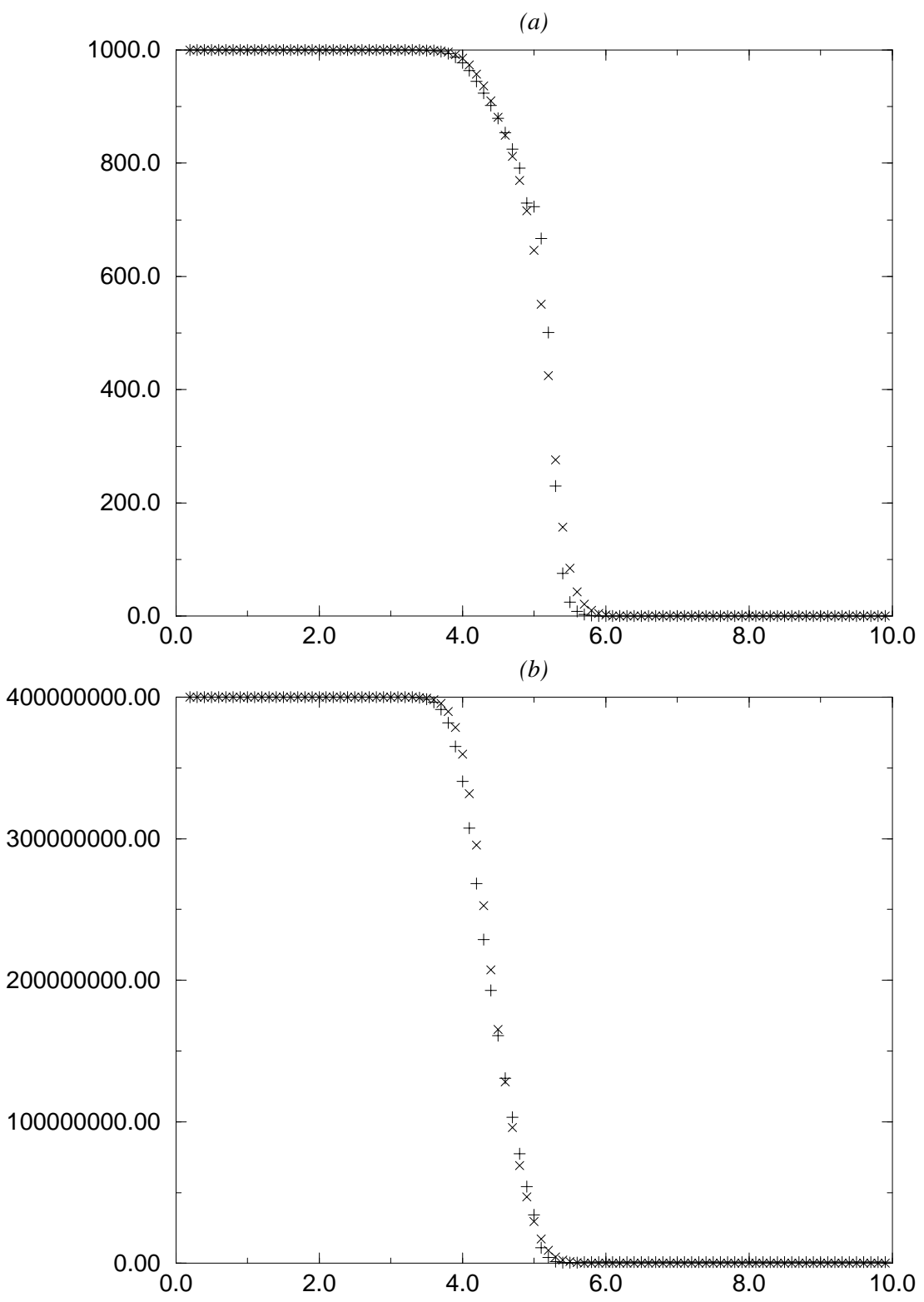

Figure 7: Case 3.7 : density (a) $-p+p_{c}$ (b) 
Rusanov 2-2

+ VFRoe ncv (Tau,u,p) 2-2
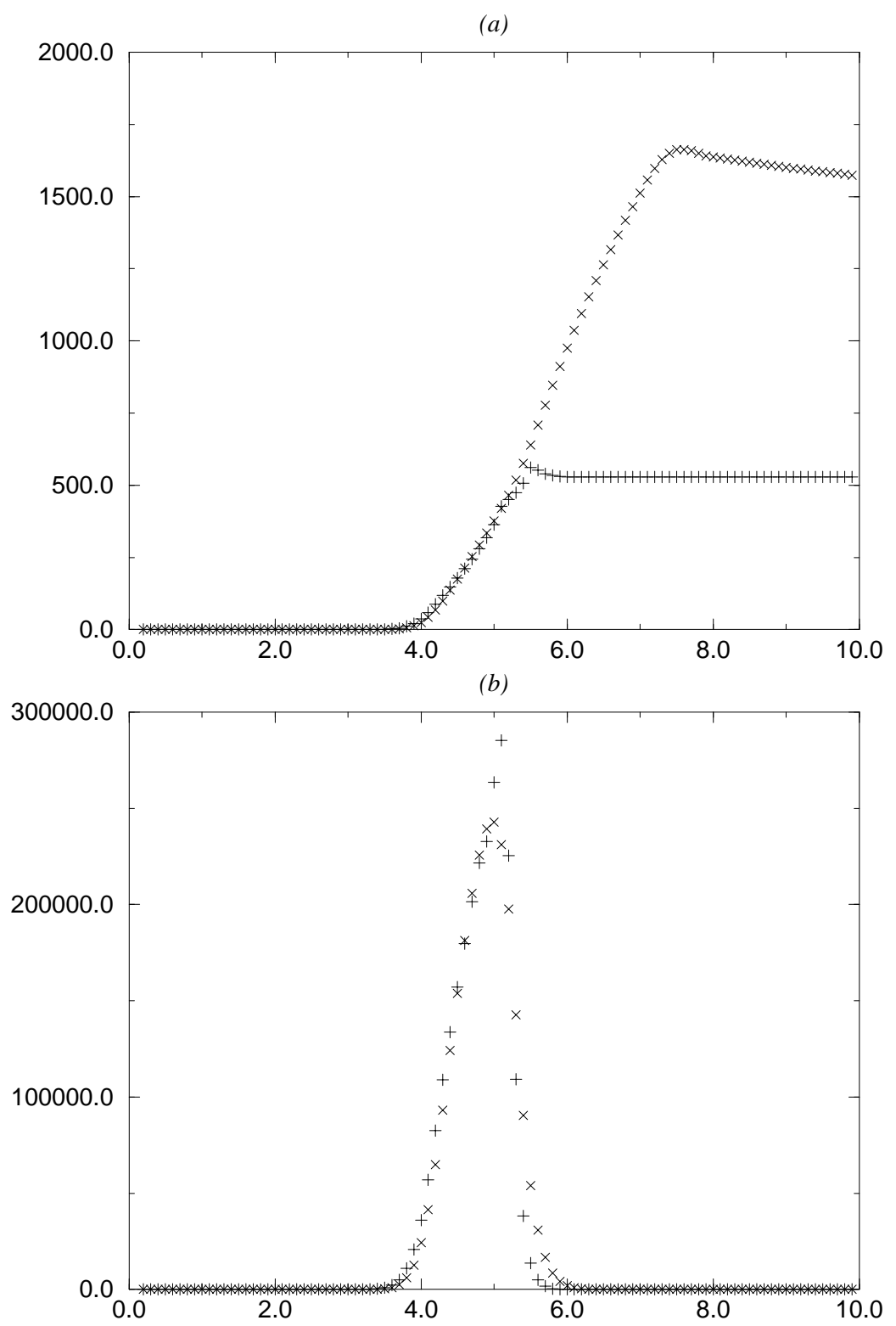

Figure 8: Case 3.7 : velocity (a) - momentum (b) 
+ VFRoe ncv (Tau,u,p) 1-1

$\times$ VFRoe ncv (Rho,u,p) 1-1

(a)

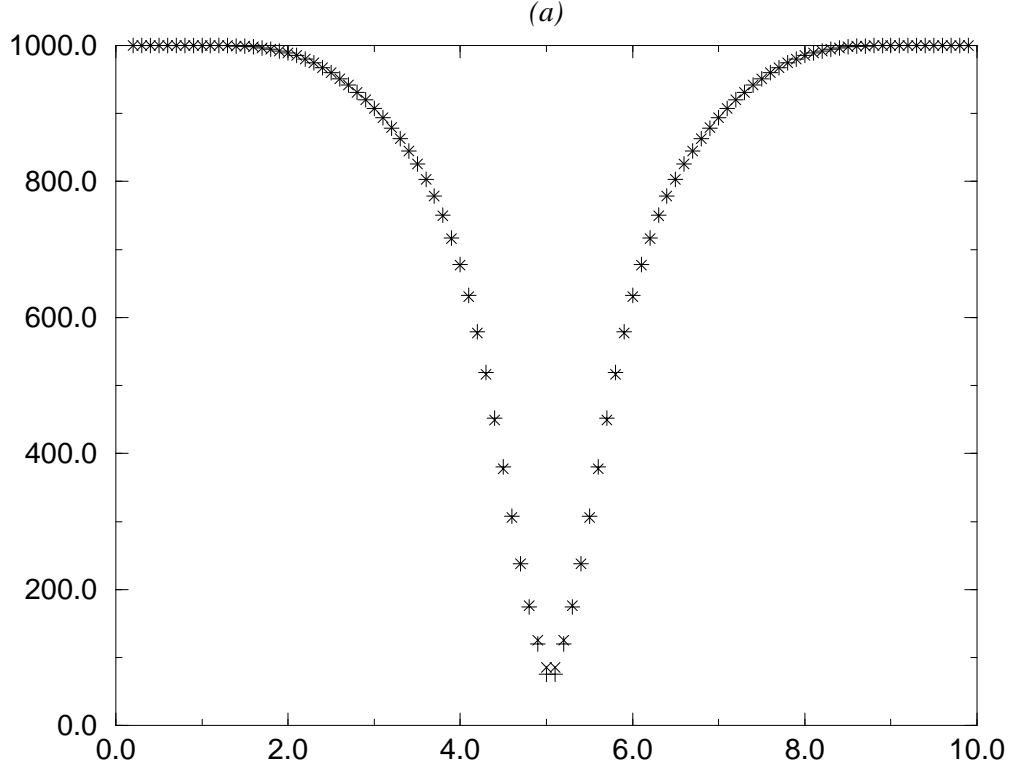

(b)

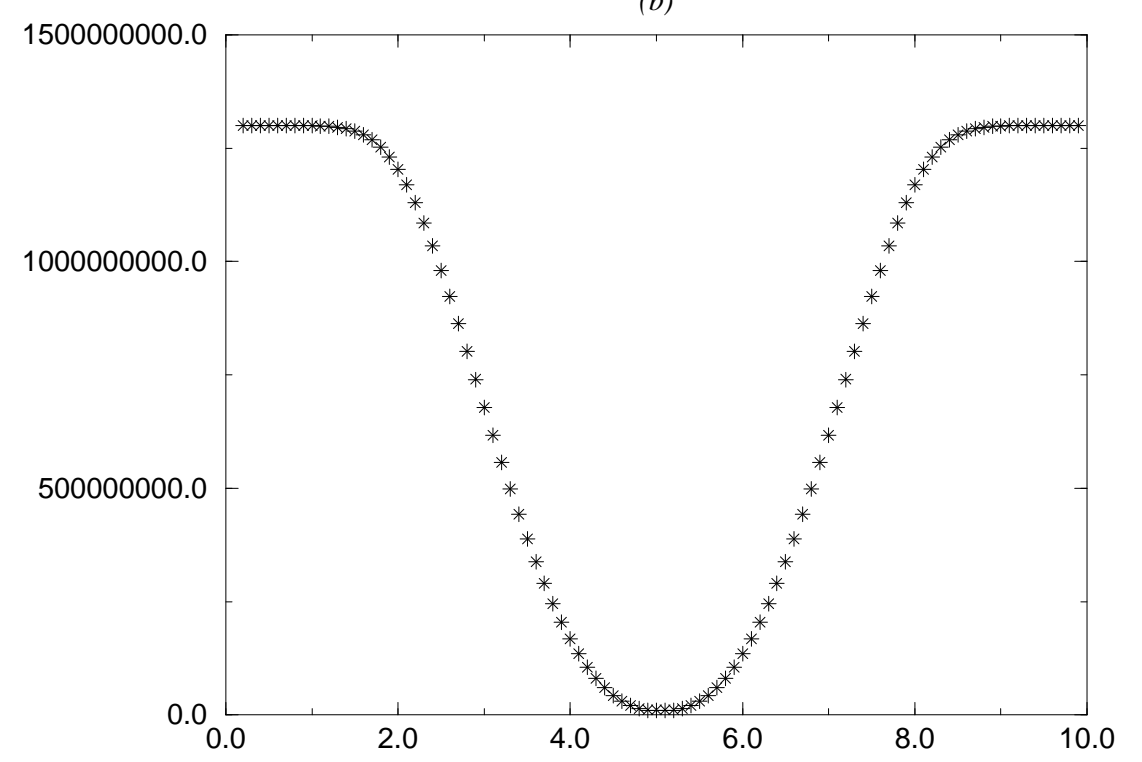

Figure 9: Case 3.8: densité (a) - $p+p_{c}$ (b) 
+ VFRoe ncv (Tau,u,p) 1-1

$\times$ VFRoe ncv (Rho,u,p) 1-1

(a)

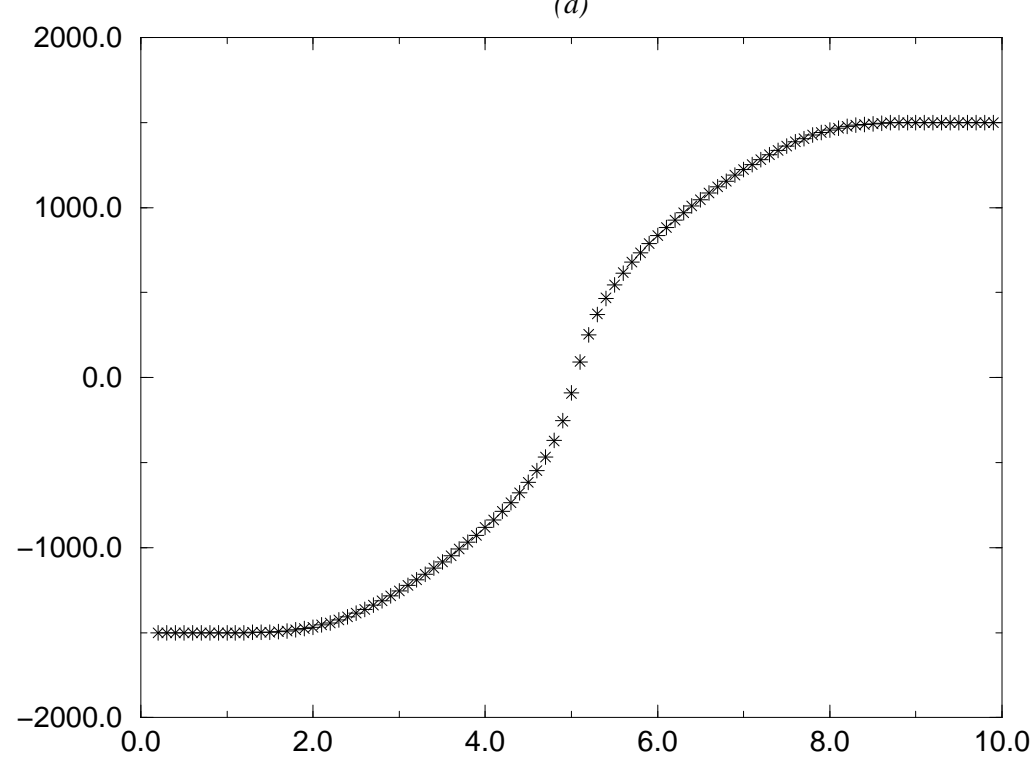

(b)

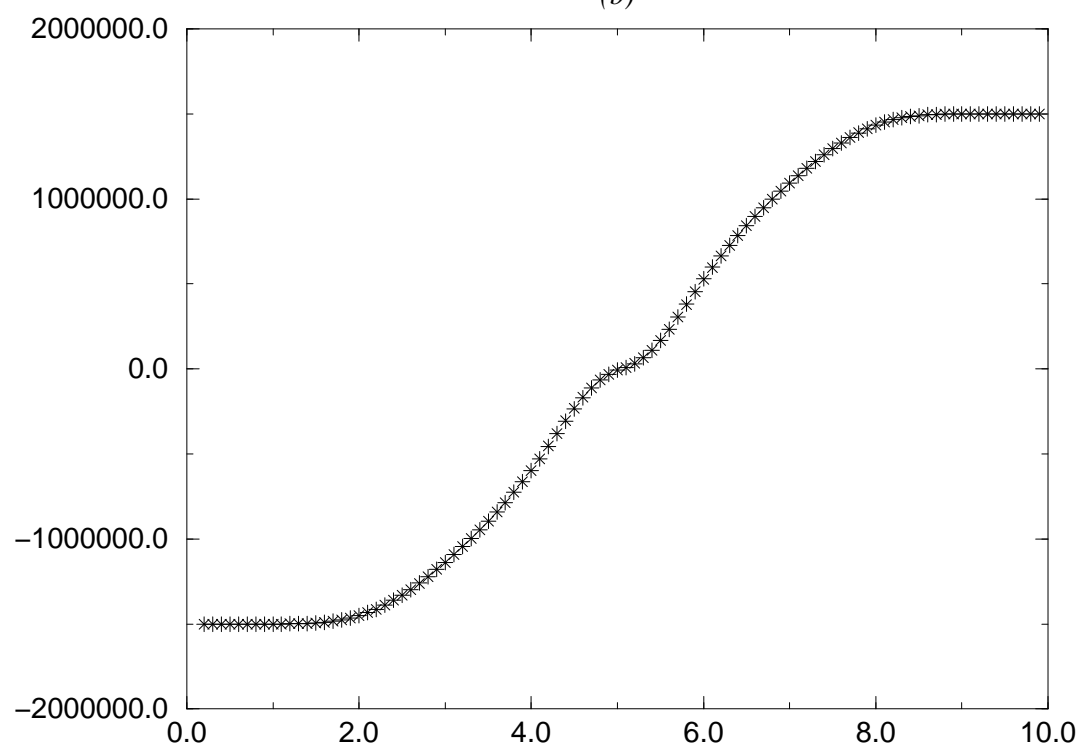

Figure 10: Case 3.8: vitesse (a) - momentum (b) 
+ VFRoe 1-1

$\times$ Rusanov 1-1

(a)

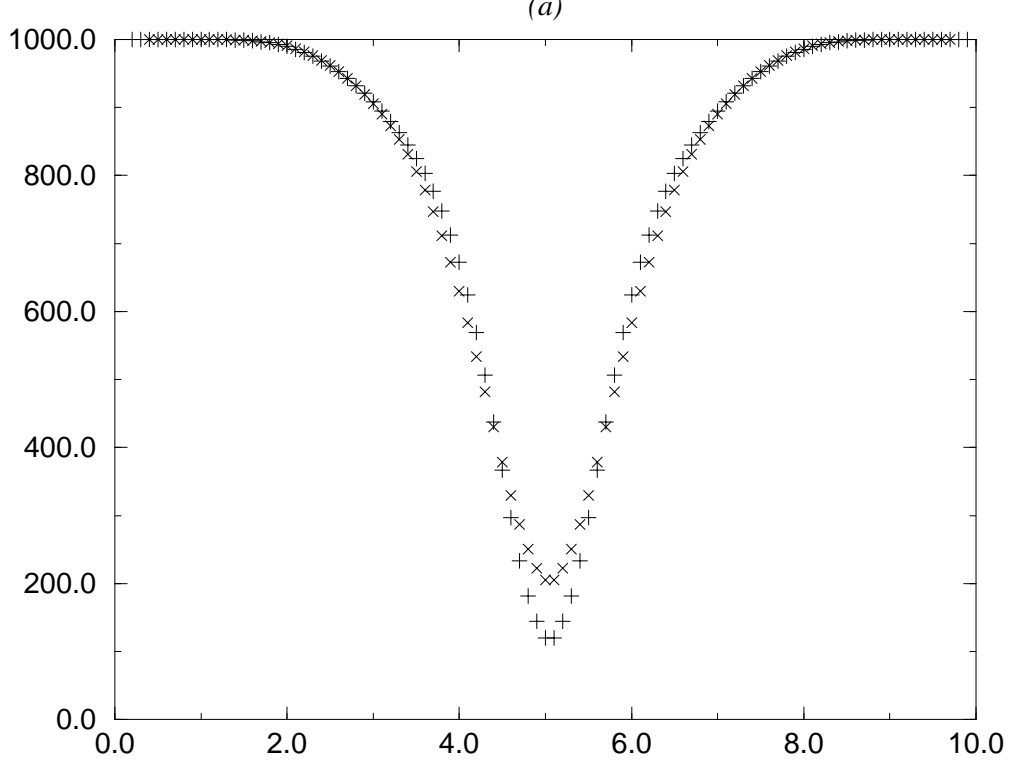

(b)

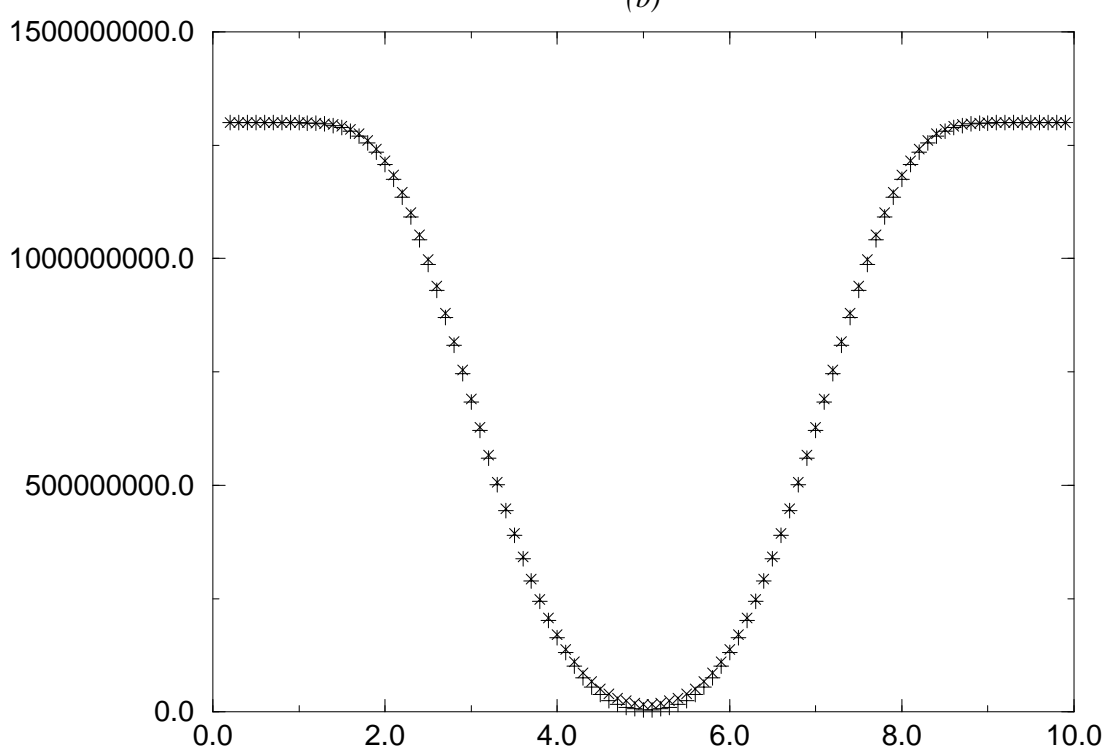

Figure 11: Case 3.8: density (a) $-p+p_{c}$ (b) 
+ VFRoe 1-1

$\times$ Rusanov 1-1

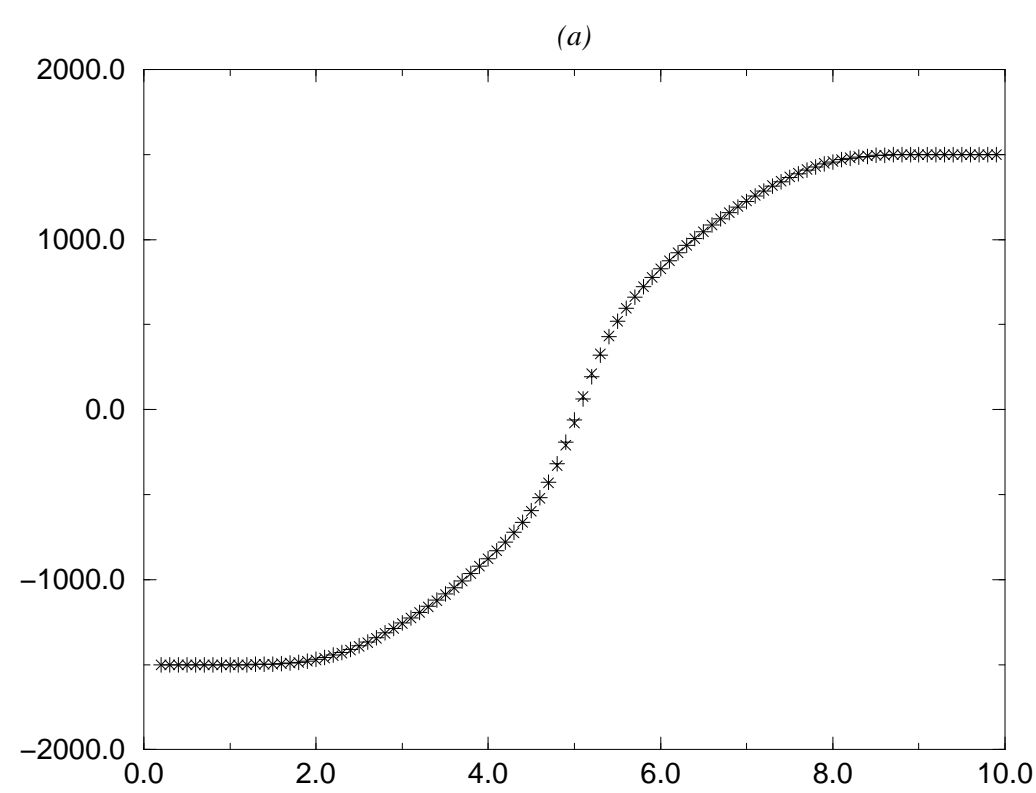

(b)

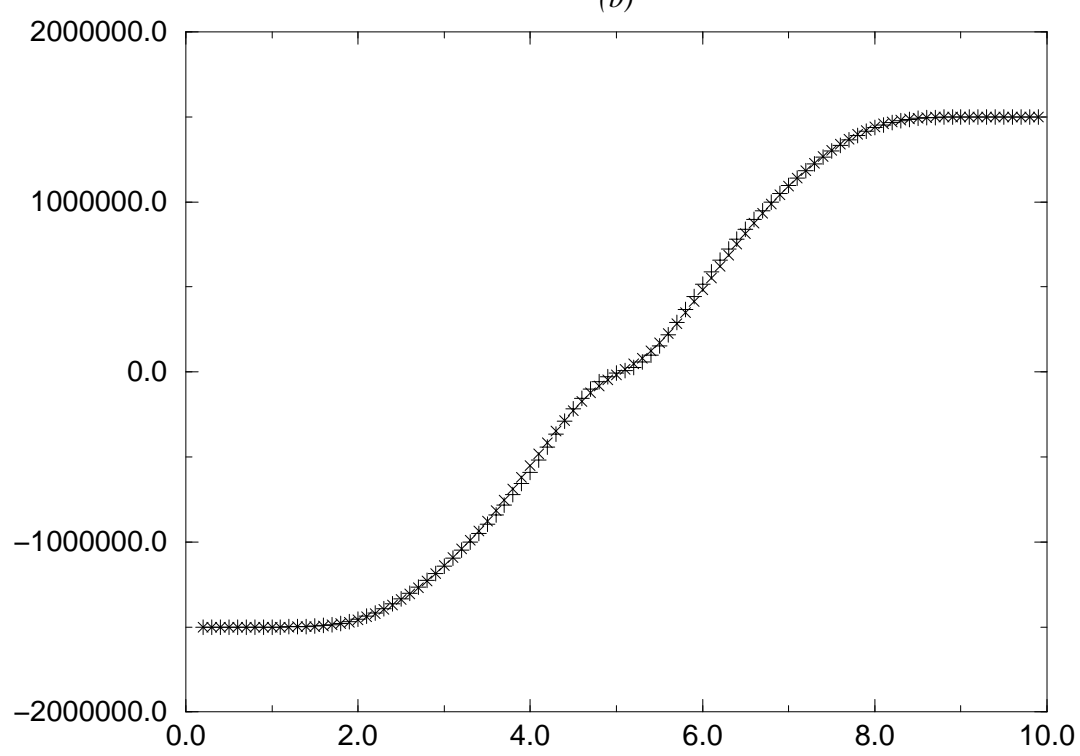

Figure 12: Case 3.8 : velocity (a) - momentum (b) 
- VFRoe ncv (Tau,u,p) 1-1 without entropy correction

+ VFRoe ncv (Tau,u,p) 1-1 + Relaxation

(a)

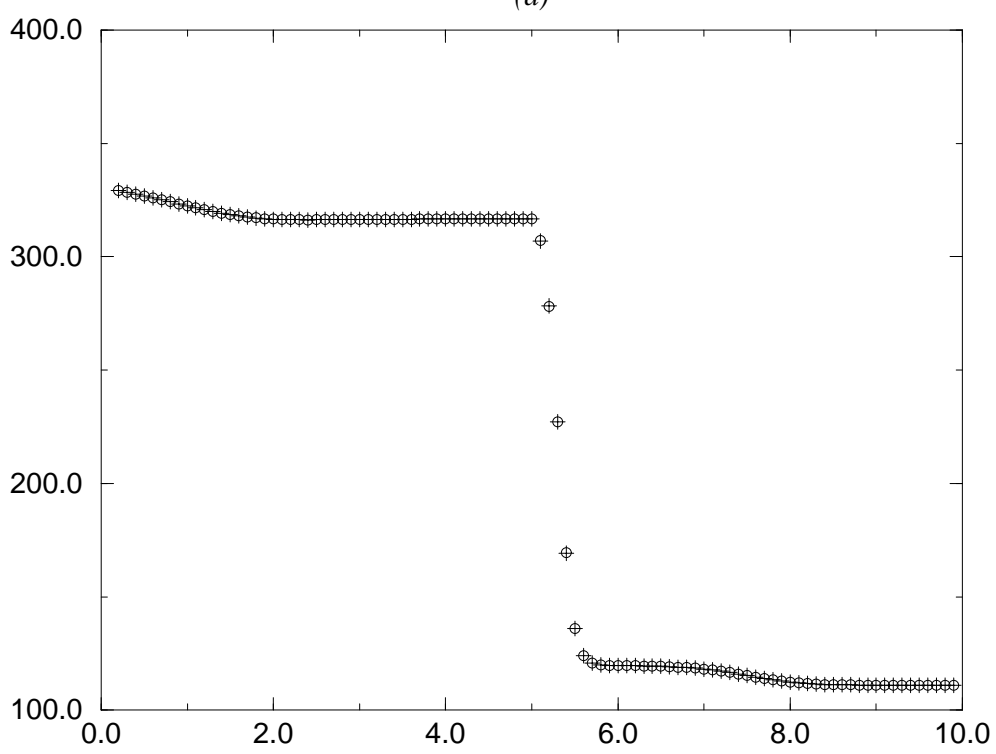

(b)

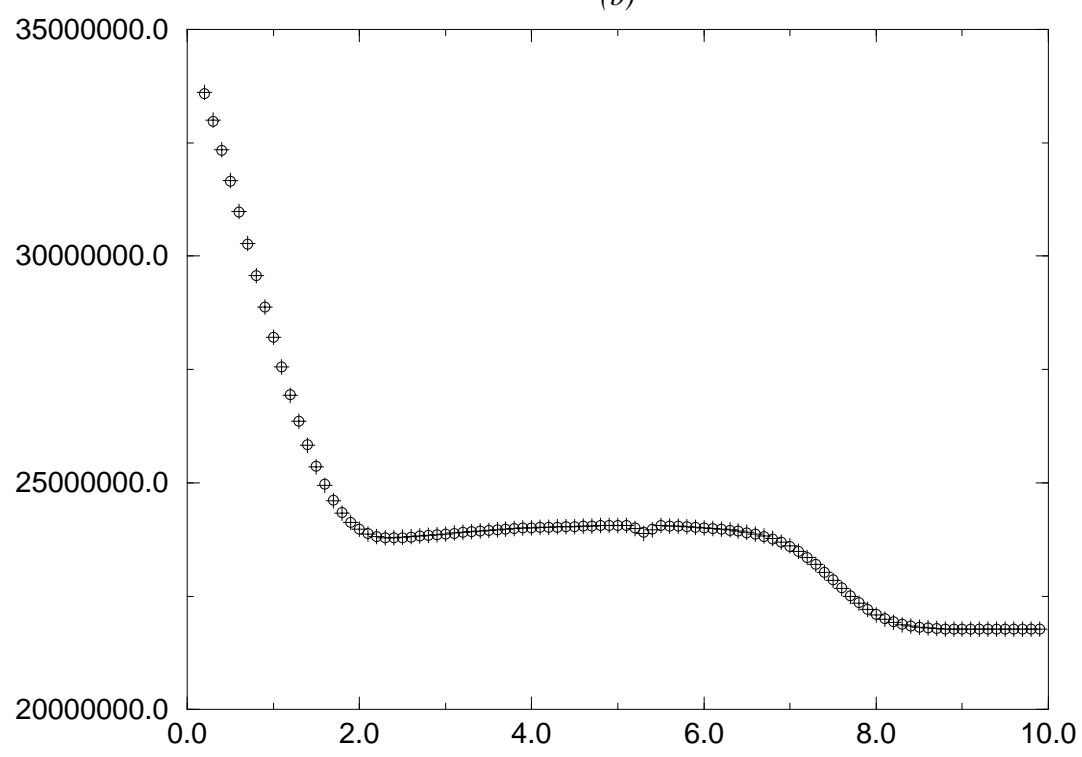

Figure 13: Case 4.1 : densité (a) $-p+p_{c}$ (b) 
- VFRoe ncv (Tau,u,p) 1-1 without entropy correction

+ VFRoe ncv (Tau,u,p) 1-1 + Relaxation

(a)

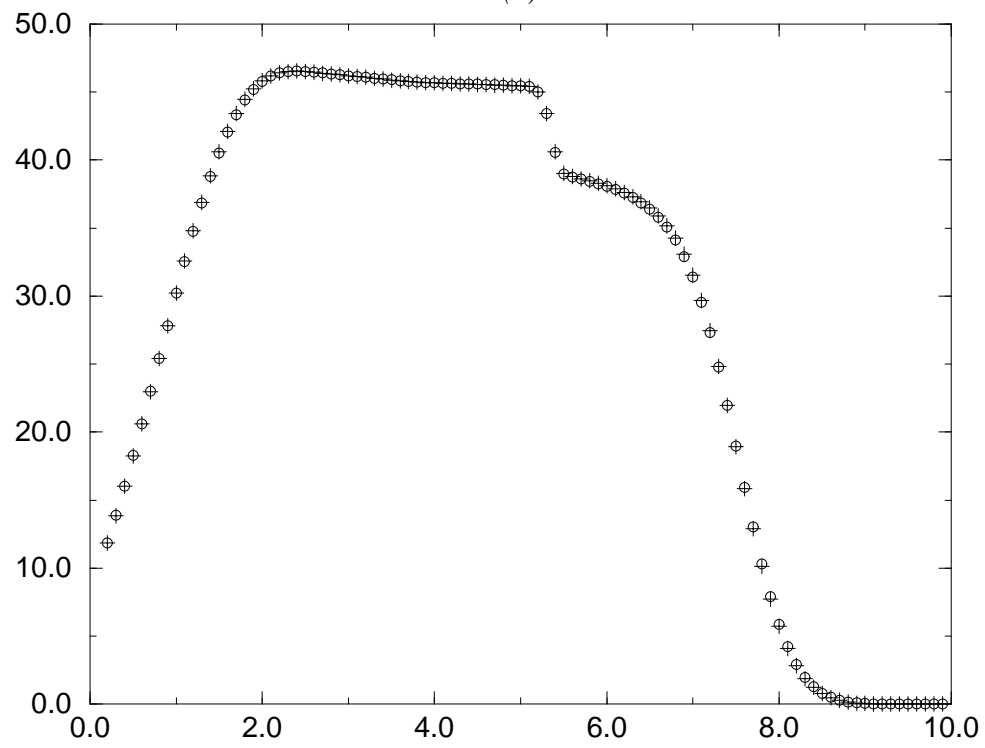

(b)

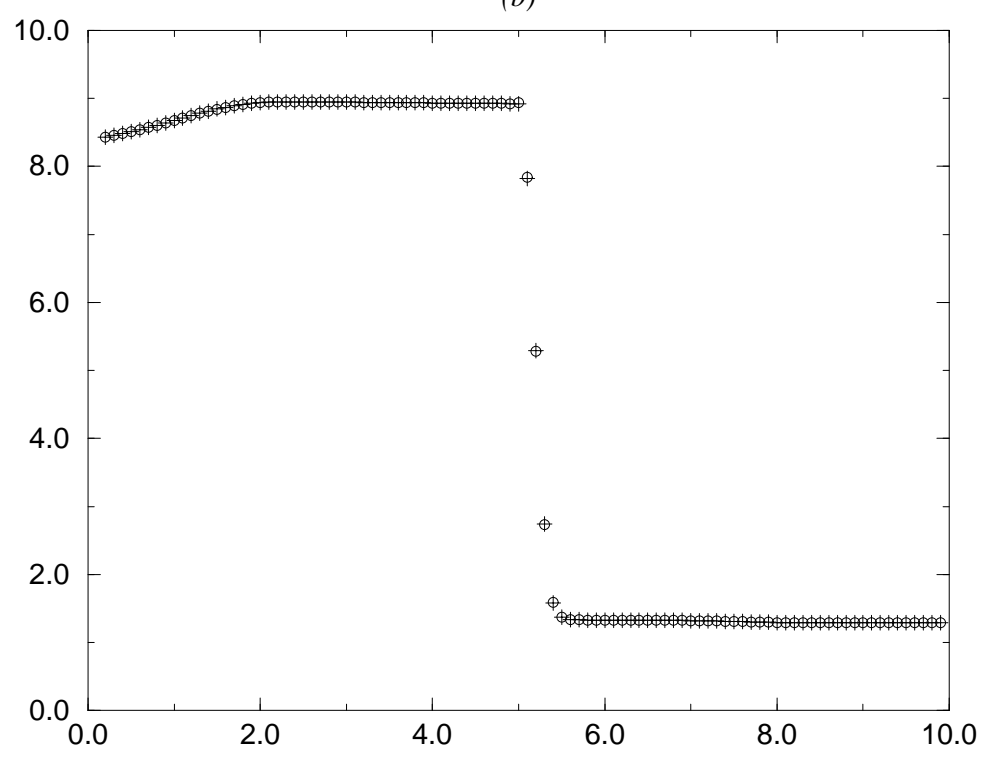

Figure 14: Case 4.1 : vitesse (a) $-\hat{\gamma}(p, \rho)$ (b) 
+ VFRoe ncv (Rho,u,p) 1-1

$\times$ VFFC 1-1

(a)

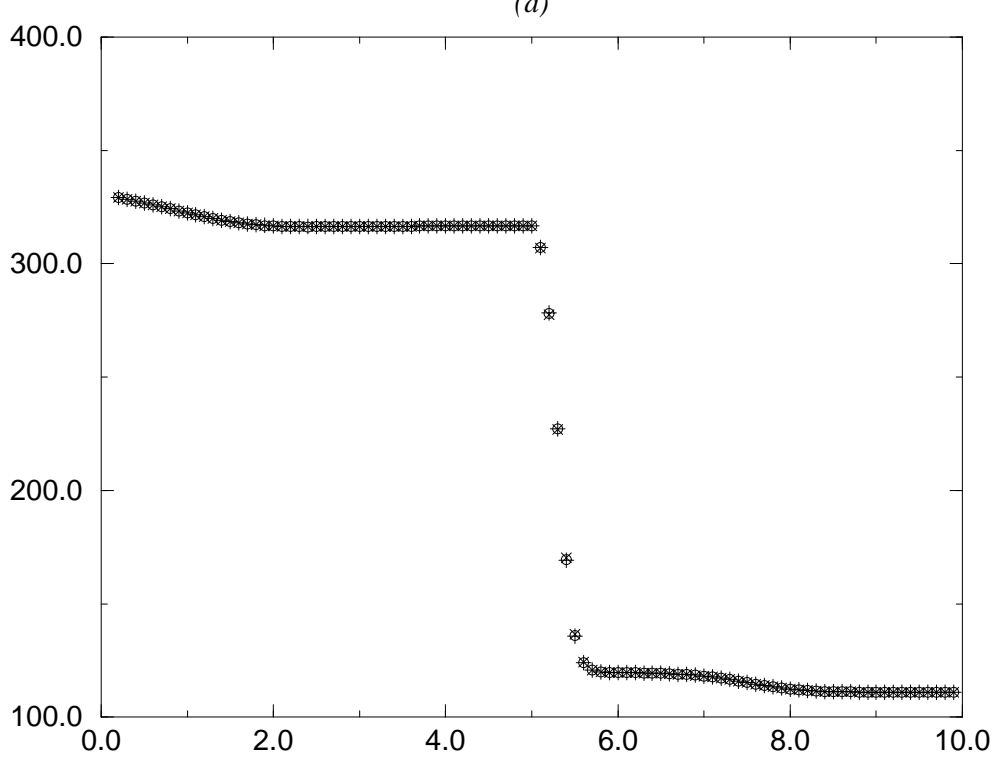

(b)

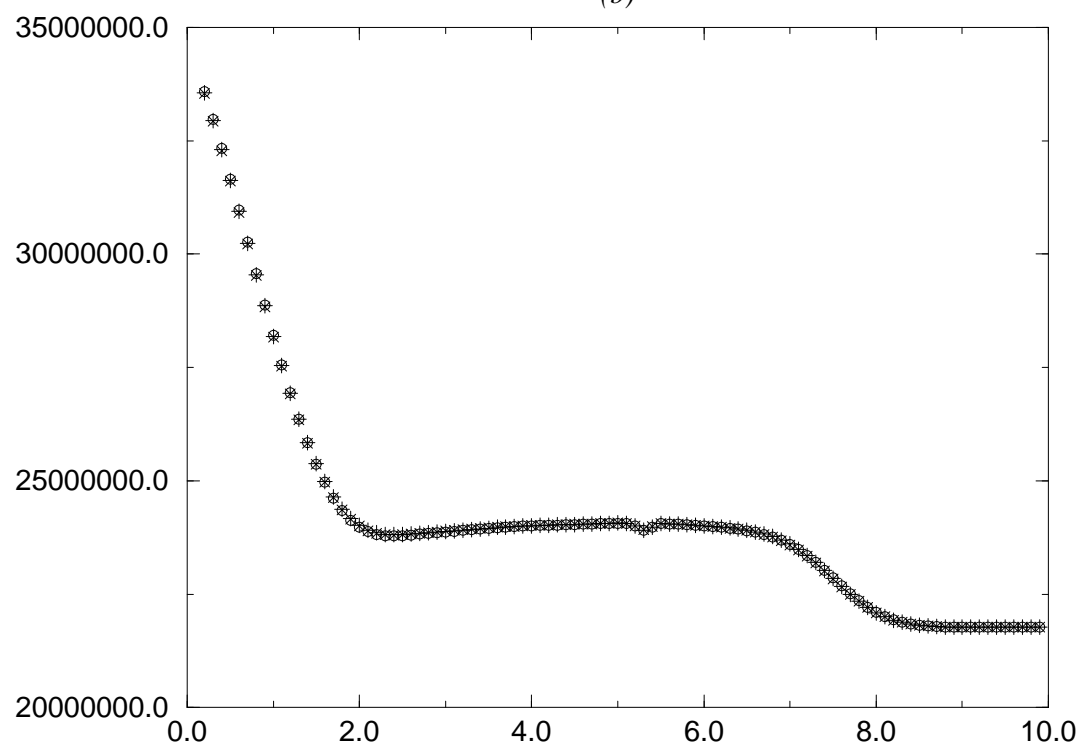

Figure 15: Case 4.1: densité (a) $-p+p_{c}$ (b) 
- VFRoe ncv (Tau,u,p) 1-1

+ VFRoe ncv (Rho,u,p) 1-1

$\times$ VFFC 1-1

(a)

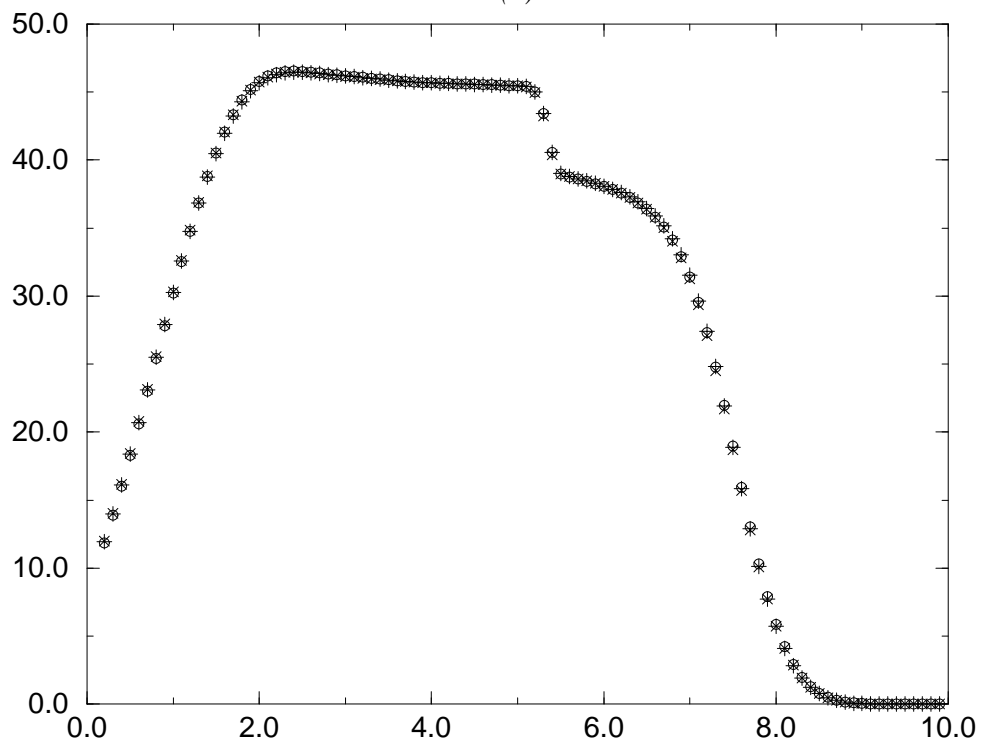

(b)

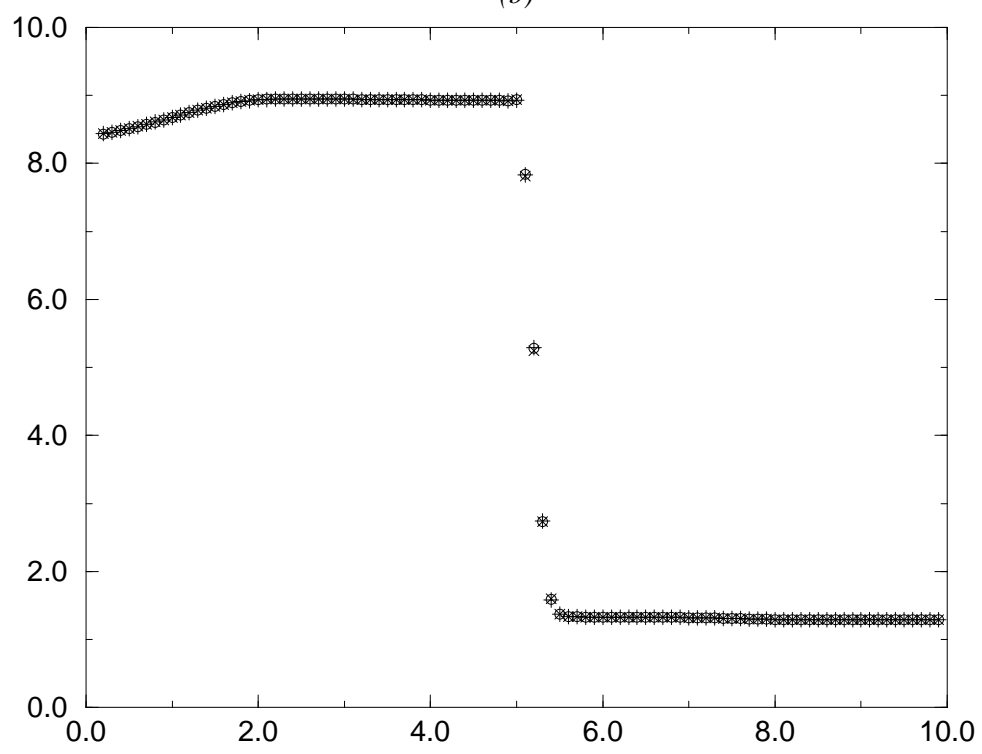

Figure 16: Case 4.1 : vitesse (a) $-\hat{\gamma}(p, \rho)$ (b) 


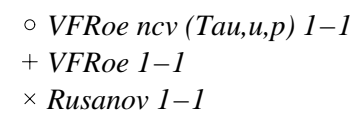

$\times$ Rusanov 1-1

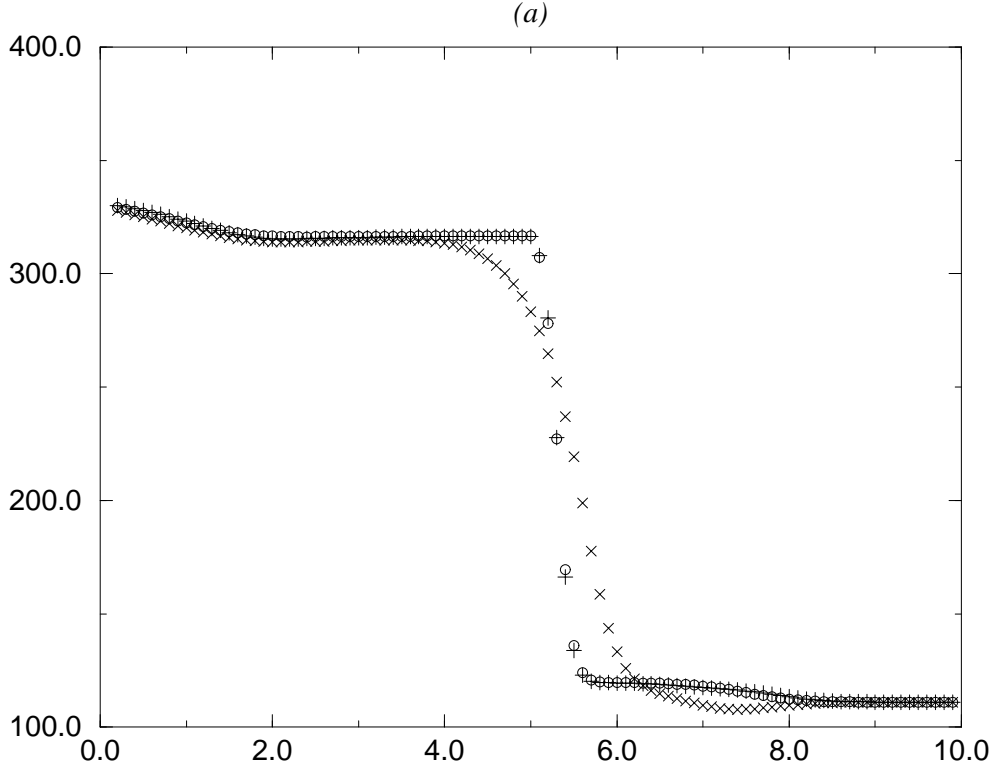

(b)

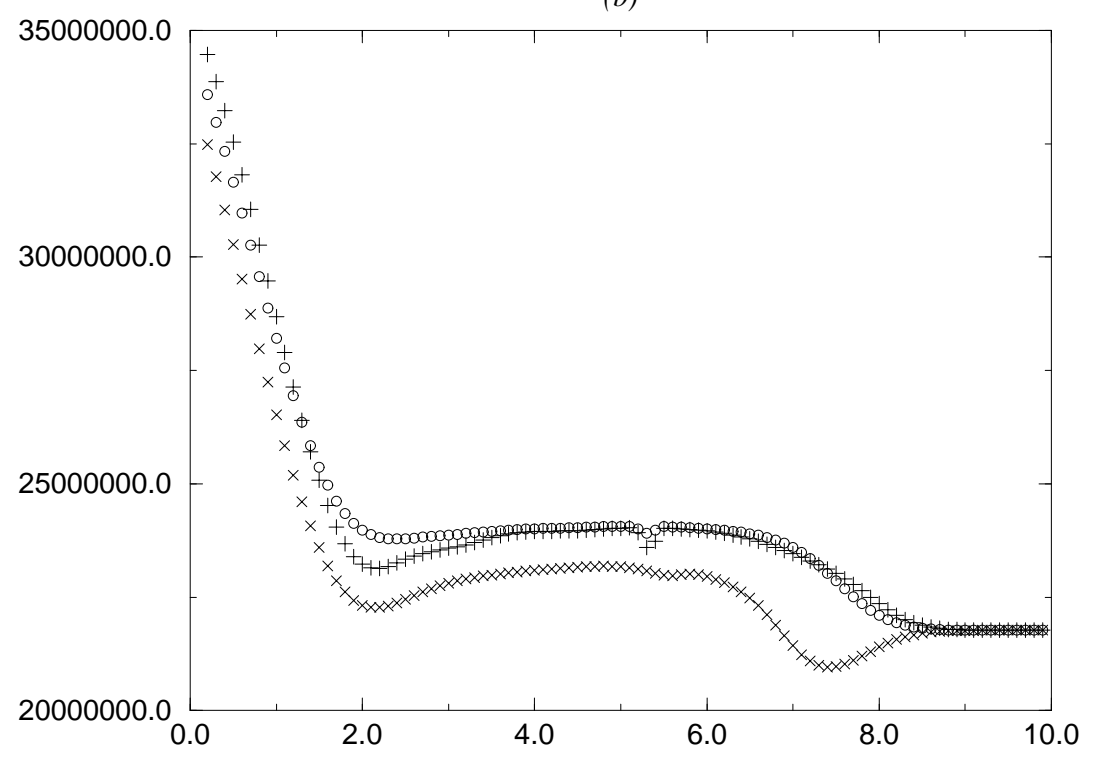

Figure 17: Case 4.1 : densité (a) $-p+p_{c}$ (b) 
- VFRoe ncv (Tau,u,p) 1-1

+ VFRoe 1-1

$\times$ Rusanov 1-1

(a)

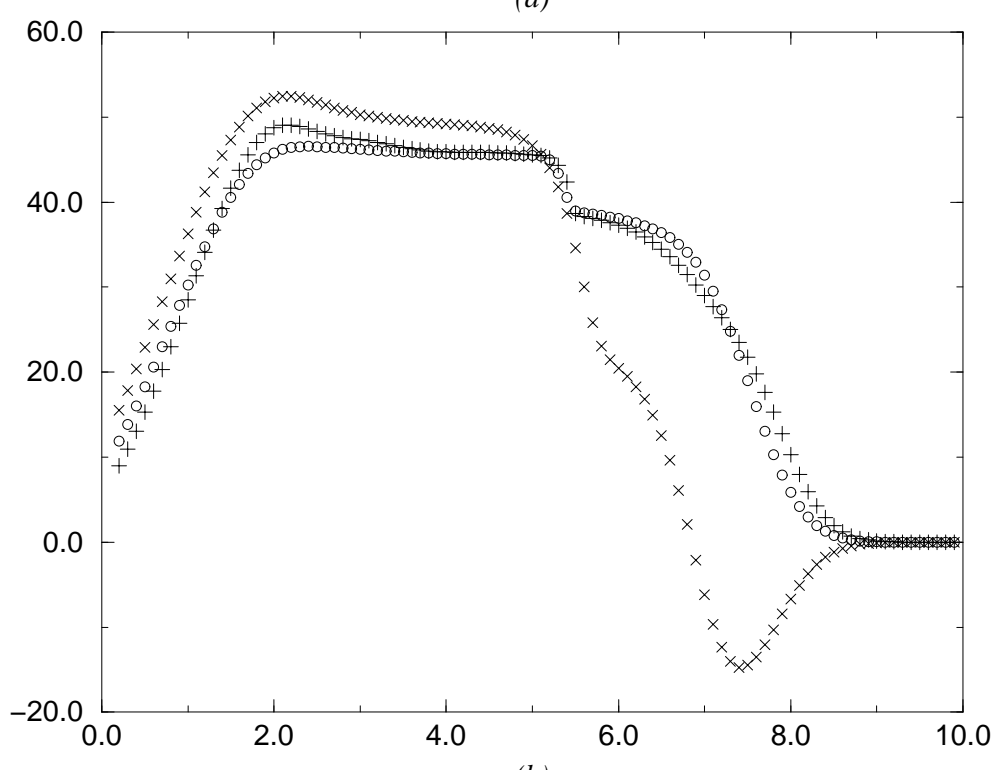

(b)

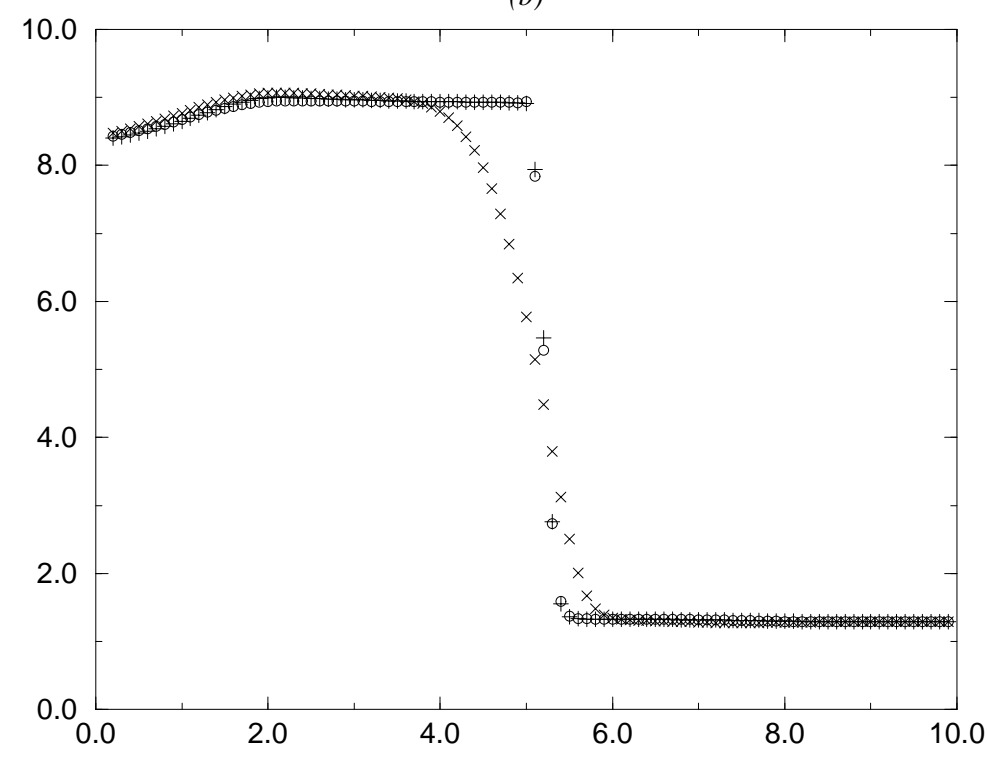

Figure 18: Case 4.1 : vitesse (a) $-\hat{\gamma}(p, \rho)$ (b) 
- VFRoe ncv (Tau,u,p) 1-1 without entropy correction

+ VFRoe ncv (Tau,u,p) 1-1 + Relaxation

(a)

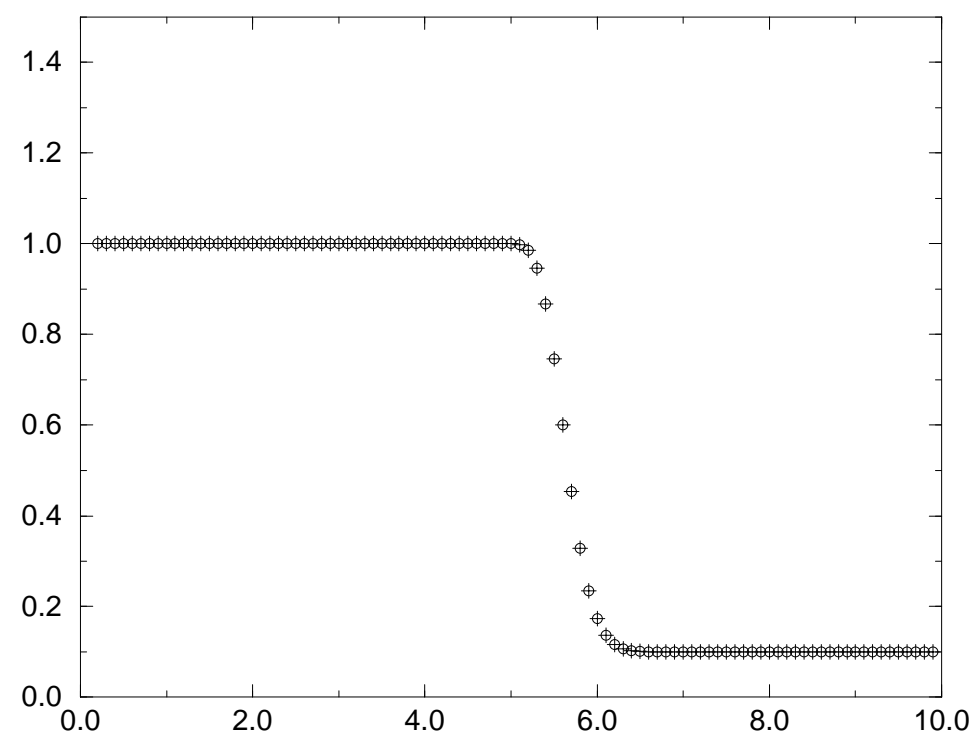

(b)

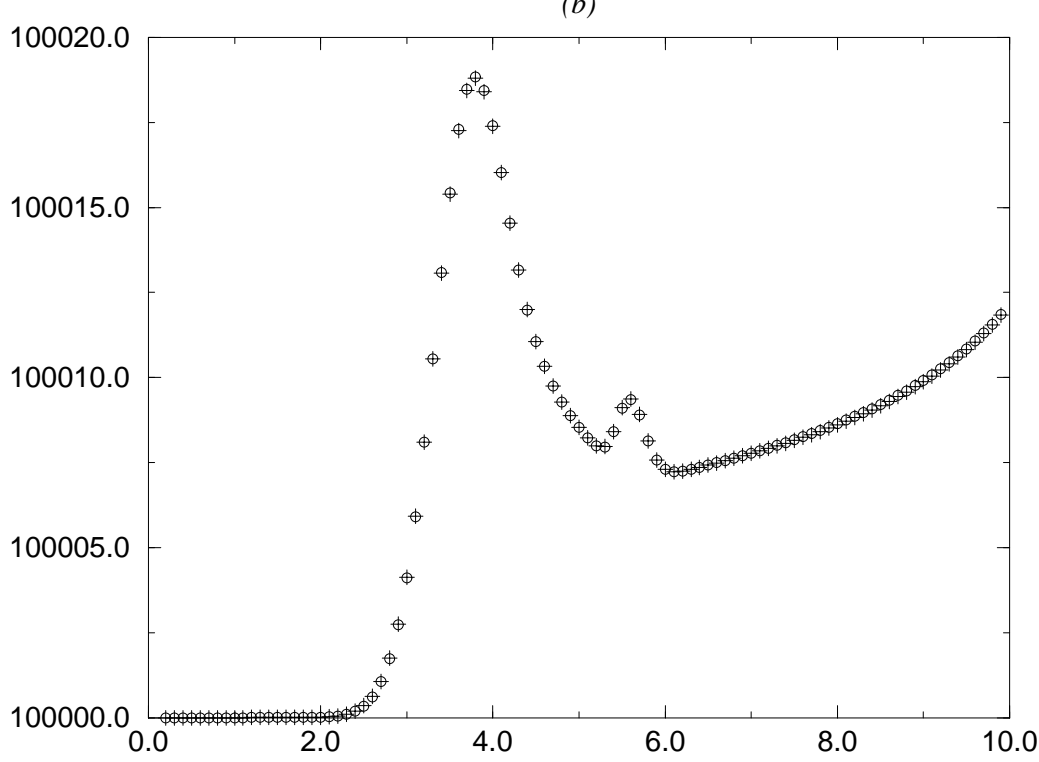

Figure 19: Case 4.2: densité (a) $-p+p_{c}$ (b) 
- VFRoe ncv (Tau,u,p) 1-1 without entropy correction

+ VFRoe ncv $($ Tau,u,p) 1-1 + Relaxation

(a)

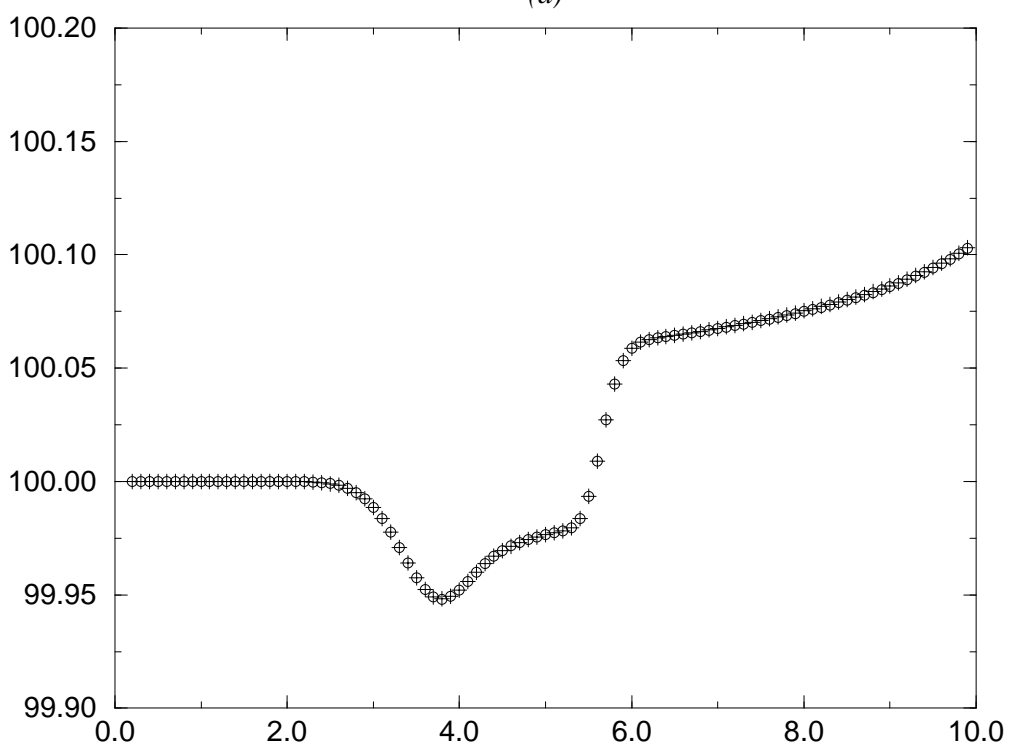

(b)

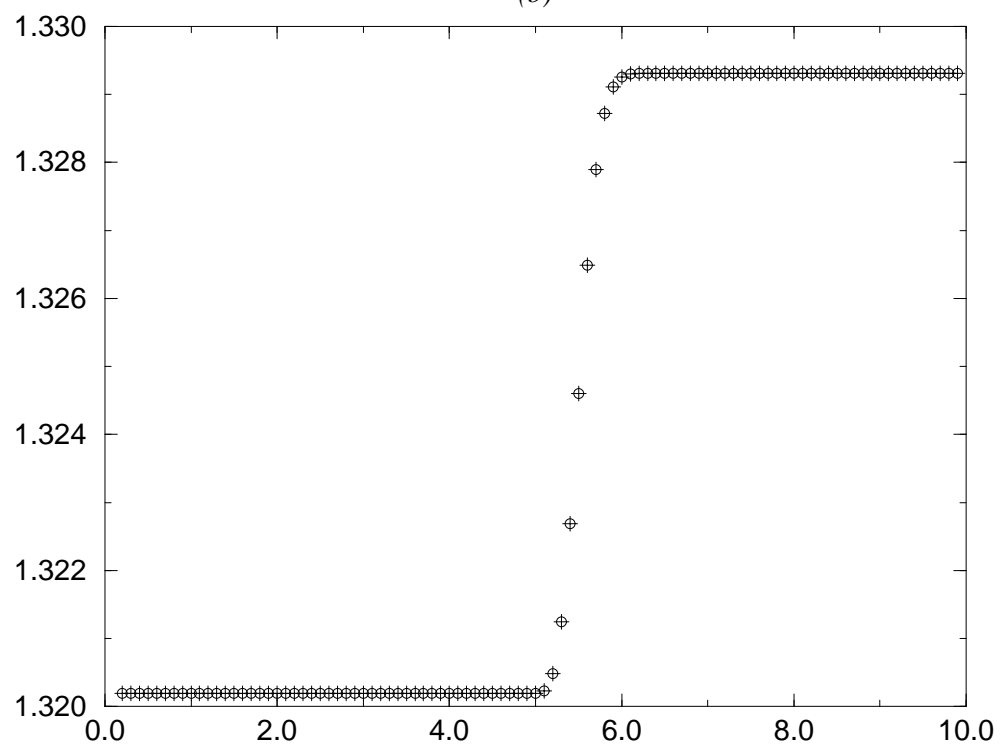

Figure 20: Case 4.2 : vitesse (a) $-\hat{\gamma}(p, \rho)$ (b) 
- VFRoe ncv (Tau,u,p) 1-1

+ VFRoe ncv (Rho,u,p) 1-1

$\times$ VFFC 1-1

(a)

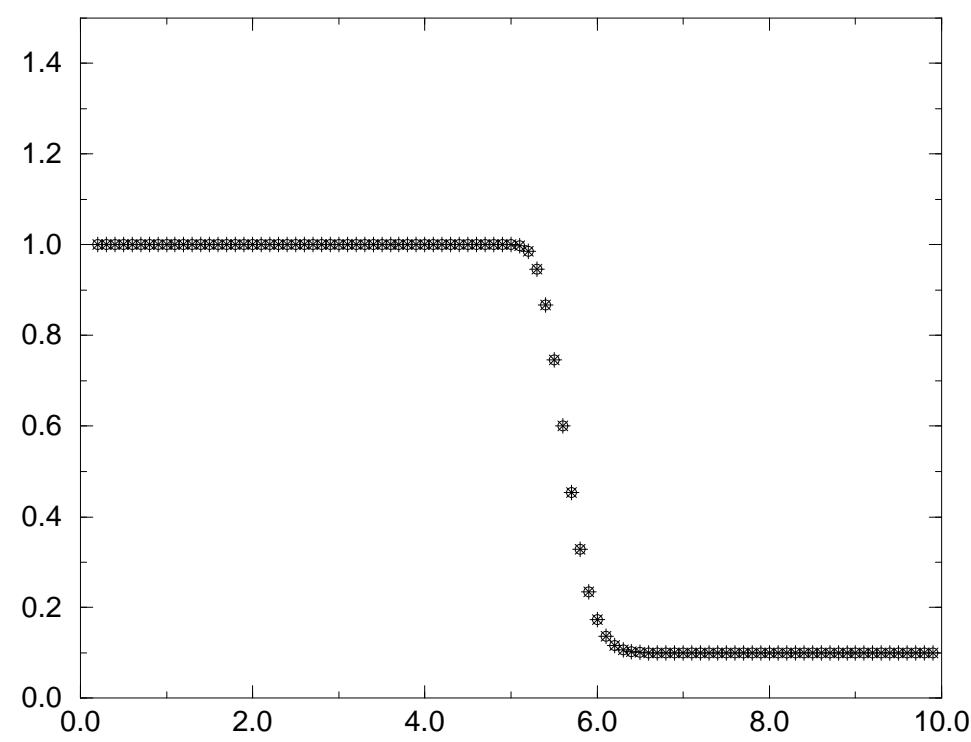

(b)

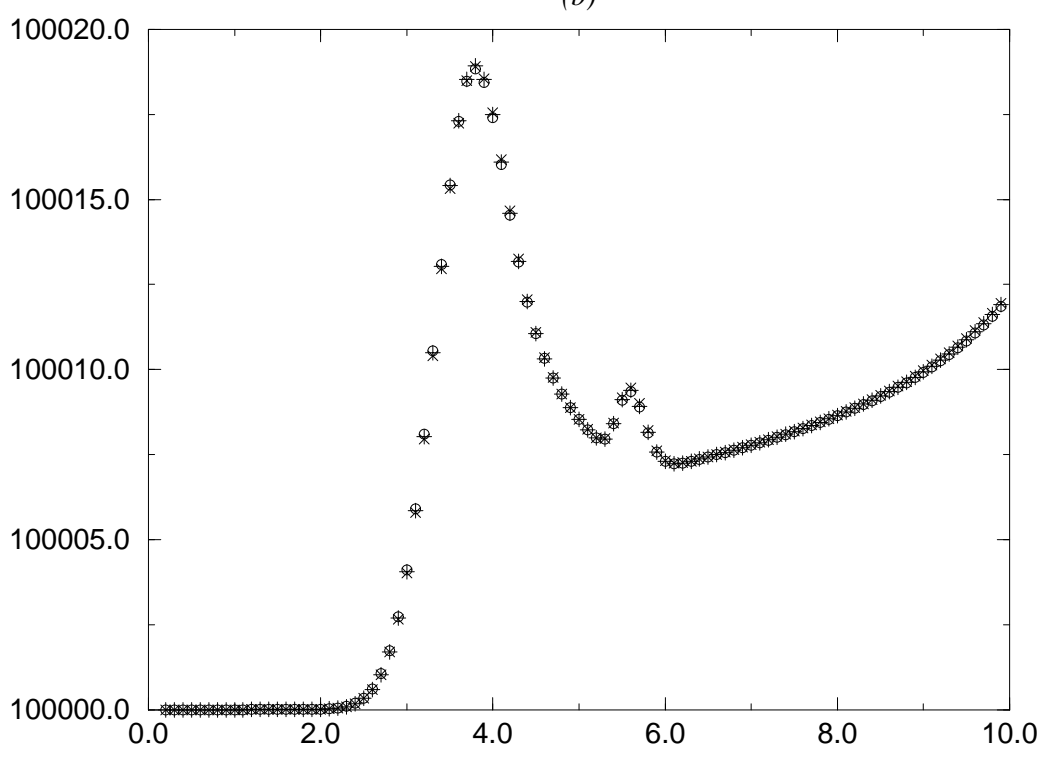

Figure 21: Case 4.2: densité (a) $-p+p_{c}$ (b) 
- VFRoe ncv (Tau,u,p) 1-1

+ VFRoe ncv (Rho,u,p) 1-1

$\times$ VFFC 1-1

(a)

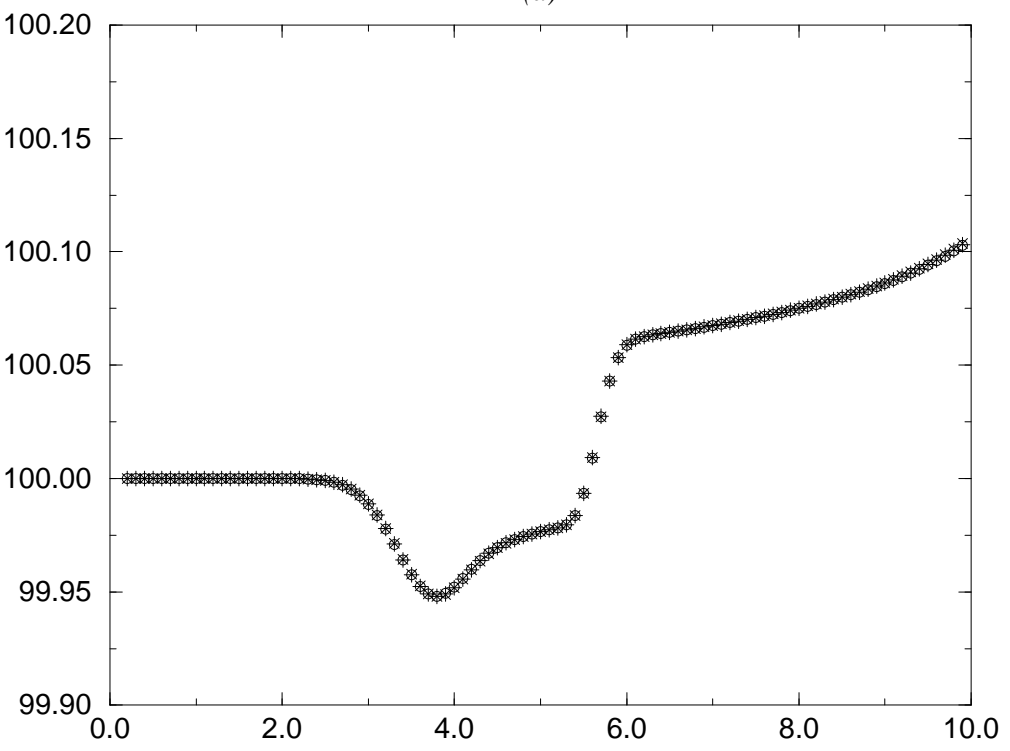

(b)

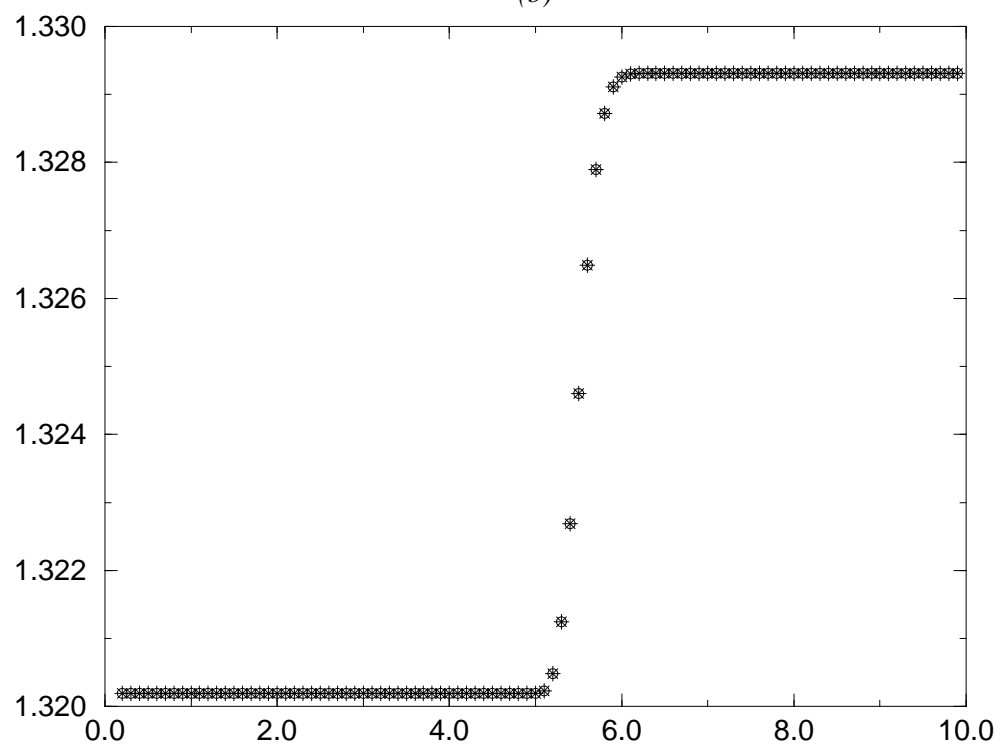

Figure 22: Case 4.2 : vitesse (a) $-\hat{\gamma}(p, \rho)$ (b) 
- VFRoe ncv (Tau,u,p) 1-1

+ VFRoe 1-1

$\times$ Rusanov 1-1

(a)

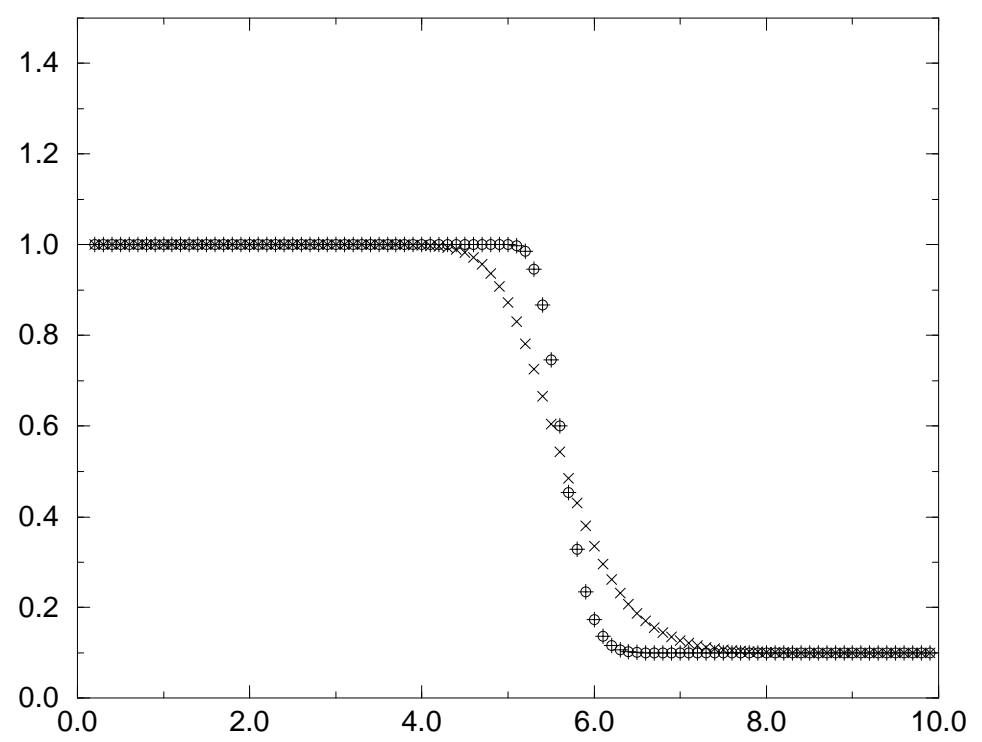

(b)

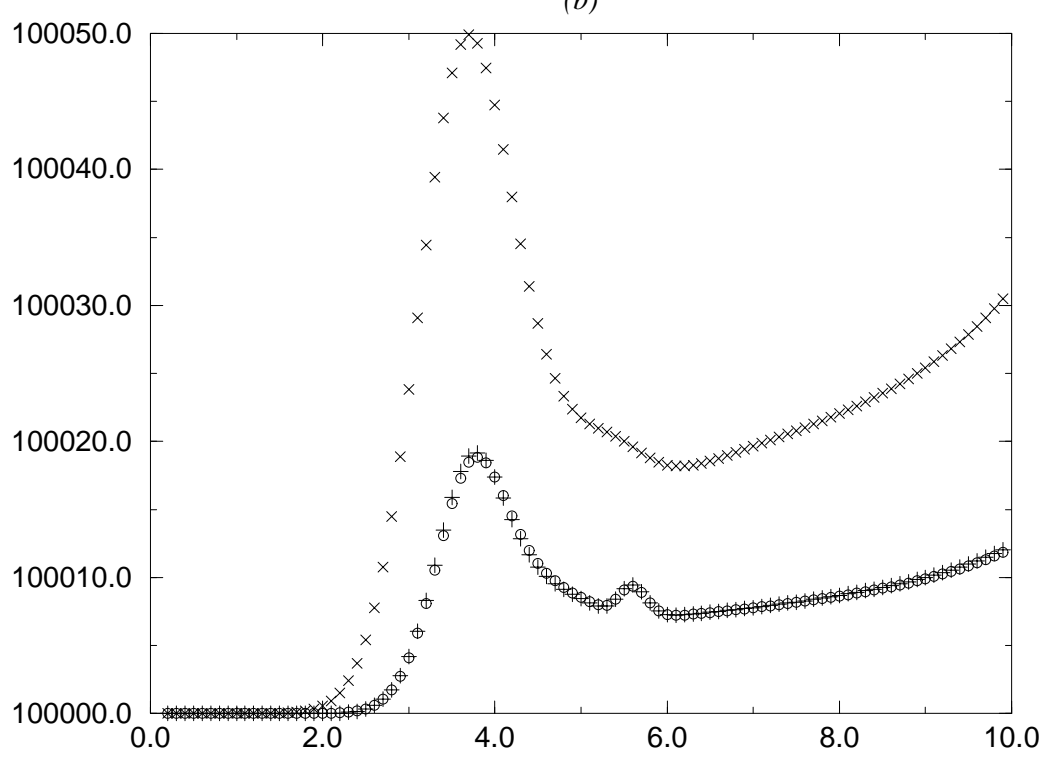

Figure 23: Case 4.2: densité (a) $-p+p_{c}$ (b) 
- VFRoe ncv (Tau,u,p) 1-1

+ VFRoe 1-1

Rusanov 1-1

(a)

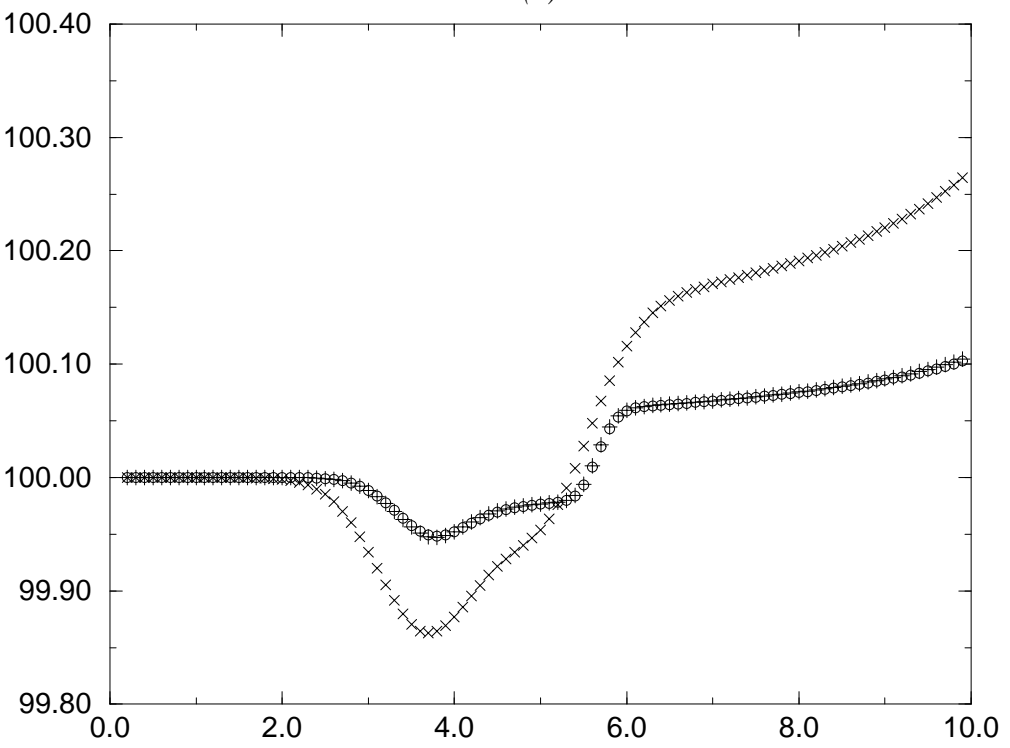

(b)

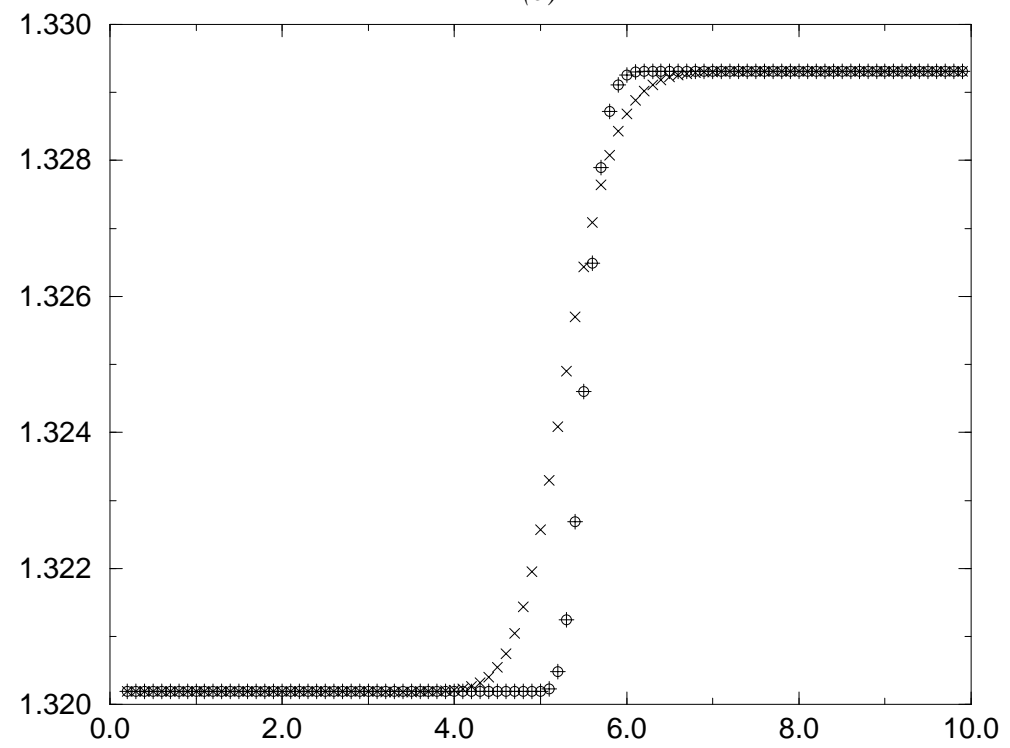

Figure 24: Case 4.2 : vitesse (a) $-\hat{\gamma}(p, \rho)$ (b) 


\subsection{Actual rates of convergence}

\section{Perfect gas EOS - Sod shock tube}

- Energy relaxation

\begin{tabular}{|c|c|c|}
\hline & 1st order & 2nd order \\
\hline$\rho$ & 0.654 & 0.791 \\
\hline$u$ & 0.853 & 0.967 \\
\hline$p$ & 0.812 & 0.988 \\
\hline
\end{tabular}

- Rusanov

\begin{tabular}{|c|c|c|}
\hline & 1st order & 2nd order \\
\hline$\rho$ & 0.651 & 0.780 \\
\hline$u$ & 0.842 & 0.970 \\
\hline$p$ & 0.823 & 0.989 \\
\hline
\end{tabular}

- $\mathrm{VFFC}$

\begin{tabular}{|c|c|c|}
\hline & 1st order & 2nd order \\
\hline$\rho$ & 0.655 & 0.792 \\
\hline$u$ & 0.855 & 0.968 \\
\hline$p$ & 0.814 & 0.988 \\
\hline
\end{tabular}

- VFRoe

\begin{tabular}{|c|c|c|}
\hline & 1st order & 2nd order \\
\hline$\rho$ & 0.654 & 0.791 \\
\hline$u$ & 0.853 & 0.967 \\
\hline$p$ & 0.811 & 0.988 \\
\hline
\end{tabular}

- VFRoe ncv $(\rho, u, p)$

\begin{tabular}{|c|c|c|}
\hline & 1st order & 2nd order \\
\hline$\rho$ & 0.654 & 0.791 \\
\hline$u$ & 0.853 & 0.967 \\
\hline$p$ & 0.811 & 0.988 \\
\hline
\end{tabular}

- VFRoe ncv $(\tau, u, p)$

\begin{tabular}{|c|c|c|}
\hline & 1st order & 2nd order \\
\hline$\rho$ & 0.653 & 0.791 \\
\hline$u$ & 0.853 & 0.967 \\
\hline$p$ & 0.812 & 0.988 \\
\hline
\end{tabular}




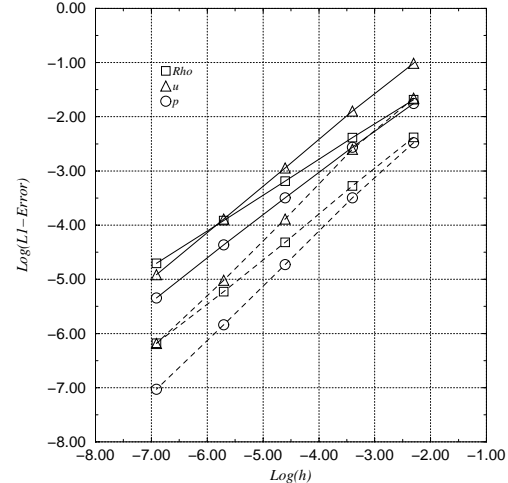

Figure 25: Relaxation d'énergie

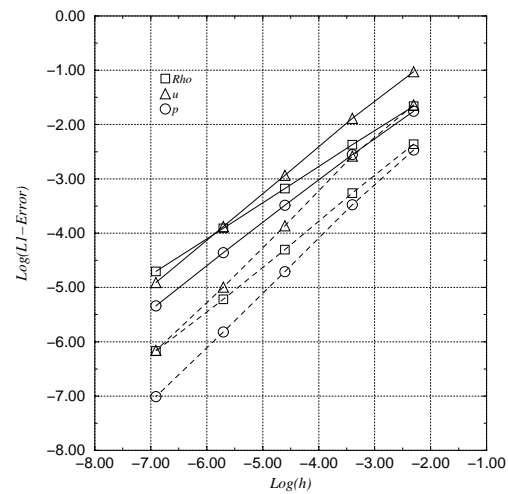

Figure 27: VFFC

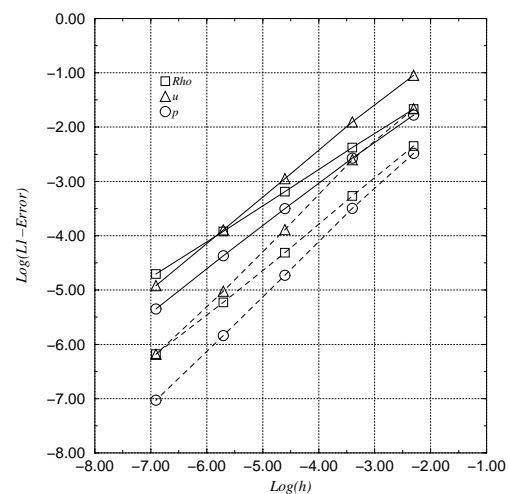

Figure 29: VFRoe ncv $(\rho, u, p)$

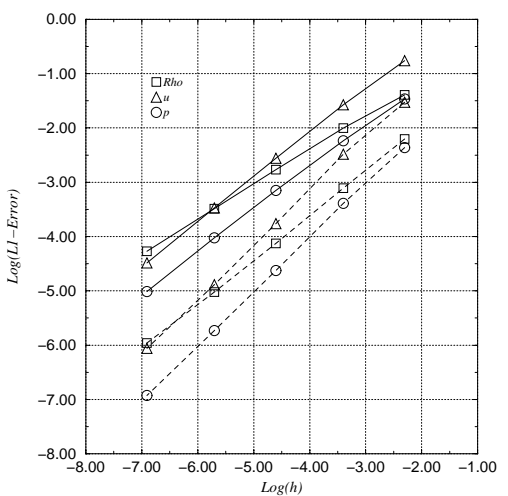

Figure 26: Rusanov

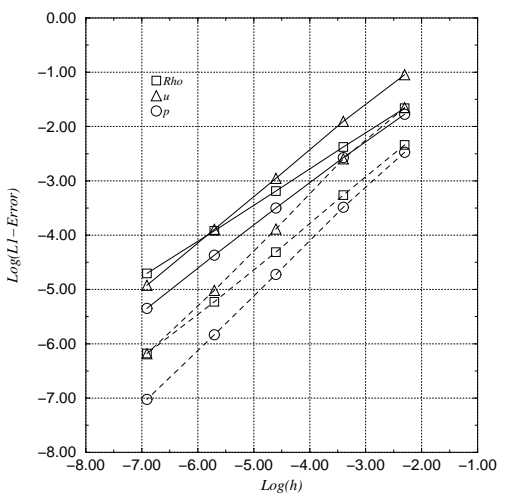

Figure 28: VFRoe

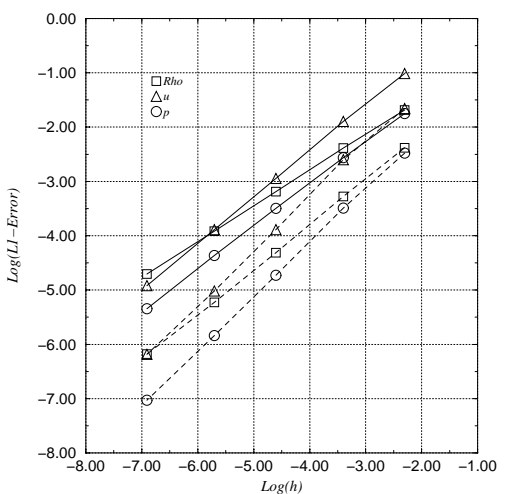

Figure 30: VFRoe ncv $(\tau, u, p)$ 
Perfect gas EOS - Sonic rarefaction wave

- Energy relaxation

\begin{tabular}{|c|c|c|}
\hline & 1st order & 2nd order \\
\hline$\rho$ & 0.890 & 0.810 \\
\hline$u$ & 0.933 & 0.973 \\
\hline$p$ & 0.927 & 0.995 \\
\hline
\end{tabular}

- Rusanov

\begin{tabular}{|c|c|c|}
\hline & 1st order & 2nd order \\
\hline$\rho$ & 0.684 & 0.827 \\
\hline$u$ & 0.794 & 0.985 \\
\hline$p$ & 0.821 & 0.999 \\
\hline
\end{tabular}

- VFFC

\begin{tabular}{|c|c|c|}
\hline & 1st order & 2nd order \\
\hline$\rho$ & 0.667 & 0.819 \\
\hline$u$ & 0.808 & 0.977 \\
\hline$p$ & 0.798 & 0.996 \\
\hline
\end{tabular}

- VFRoe

\begin{tabular}{|c|c|c|}
\hline & 1st order & 2nd order \\
\hline$\rho$ & 0.669 & 0.828 \\
\hline$u$ & 0.791 & 0.975 \\
\hline$p$ & 0.796 & 0.996 \\
\hline
\end{tabular}

- VFRoe ncv $(\rho, u, p)$

\begin{tabular}{|c|c|c|}
\hline & 1st order & 2nd order \\
\hline$\rho$ & 0.667 & 0.840 \\
\hline$u$ & 0.805 & 0.977 \\
\hline$p$ & 0.796 & 0.995 \\
\hline
\end{tabular}

- VFRoe ncv $(\tau, u, p)$

\begin{tabular}{|c|c|c|}
\hline & 1st order & 2nd order \\
\hline$\rho$ & 0.653 & 0.809 \\
\hline$u$ & 0.822 & 0.973 \\
\hline$p$ & 0.802 & 0.995 \\
\hline
\end{tabular}




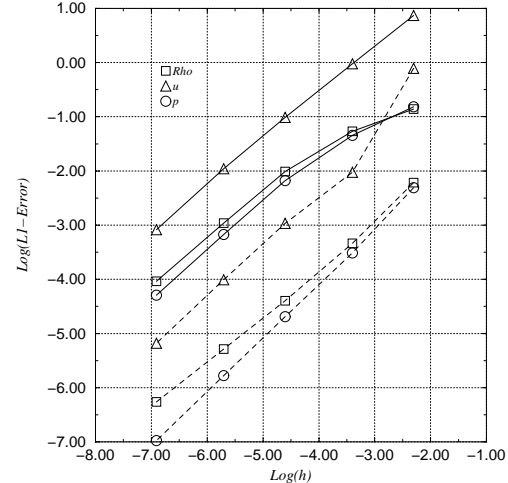

Figure 31: Relaxation d'énergie

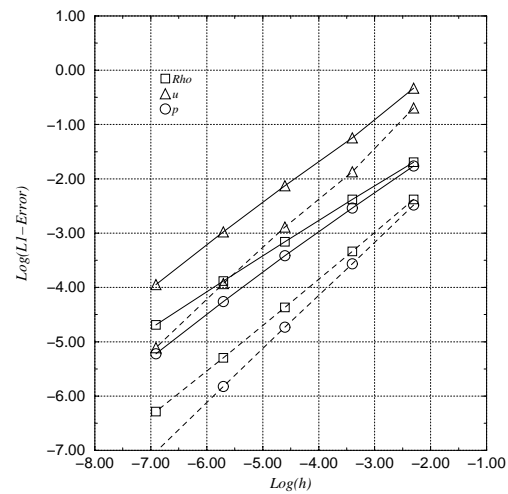

Figure 33: VFFC

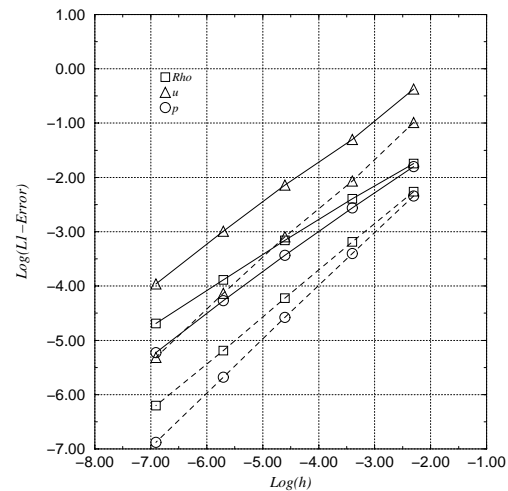

Figure 35: VFRoe ncv $(\rho, u, p)$

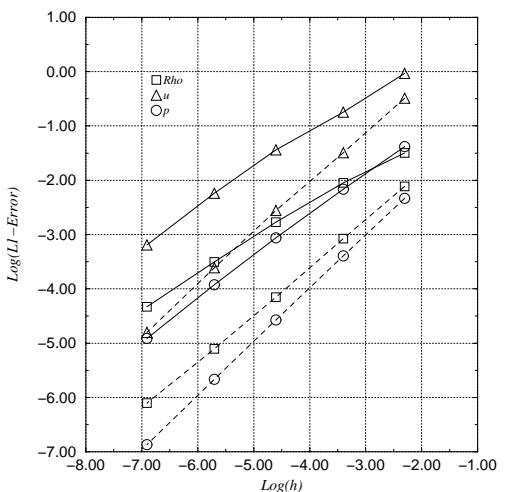

Figure 32: Rusanov

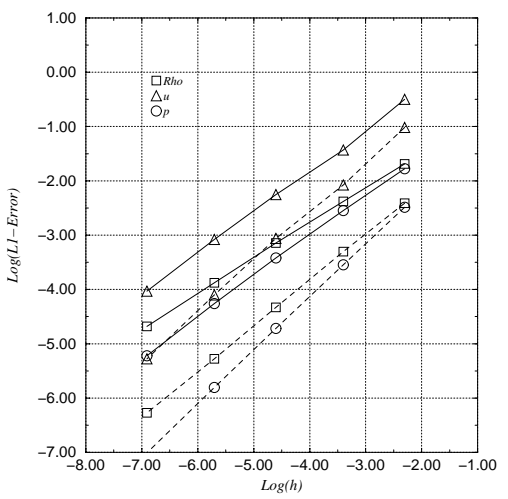

Figure 34: VFRoe

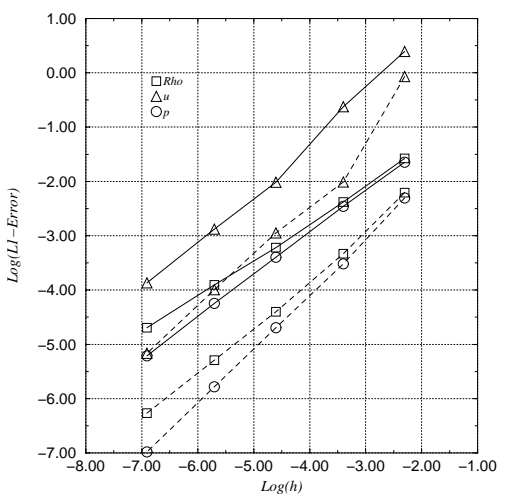

Figure 36: VFRoe ncv $(\tau, u, p)$ 
Perfect gas EOS - Symmetrical double rarefaction wave

- Energy relaxation

\begin{tabular}{|c|c|c|}
\hline & 1st order & 2nd order \\
\hline$\rho$ & 0.771 & 0.998 \\
\hline$u$ & 0.785 & 0.999 \\
\hline$p$ & 0.775 & 0.999 \\
\hline
\end{tabular}

- Rusanov

\begin{tabular}{|c|c|c|}
\hline & 1st order & 2nd order \\
\hline$\rho$ & 0.773 & 0.999 \\
\hline$u$ & 0.787 & 1.000 \\
\hline$p$ & 0.777 & 0.999 \\
\hline
\end{tabular}

- VFFC

\begin{tabular}{|c|c|c|}
\hline & 1st order & 2nd order \\
\hline$\rho$ & 0.768 & 0.998 \\
\hline$u$ & 0.782 & 1.000 \\
\hline$p$ & 0.772 & 0.999 \\
\hline
\end{tabular}

- VFRoe

\begin{tabular}{|c|c|c|}
\hline & 1st order & 2nd order \\
\hline$\rho$ & 0.771 & 0.998 \\
\hline$u$ & 0.785 & 0.999 \\
\hline$p$ & 0.775 & 0.999 \\
\hline
\end{tabular}

- VFRoe ncv $(\rho, u, p)$

\begin{tabular}{|c|c|c|}
\hline & 1st order & 2nd order \\
\hline$\rho$ & 0.771 & 0.998 \\
\hline$u$ & 0.785 & 0.999 \\
\hline$p$ & 0.775 & 0.999 \\
\hline
\end{tabular}

- VFRoe ncv $(\tau, u, p)$

\begin{tabular}{|c|c|c|}
\hline & 1st order & 2nd order \\
\hline$\rho$ & 0.771 & 0.998 \\
\hline$u$ & 0.785 & 0.999 \\
\hline$p$ & 0.775 & 0.999 \\
\hline
\end{tabular}




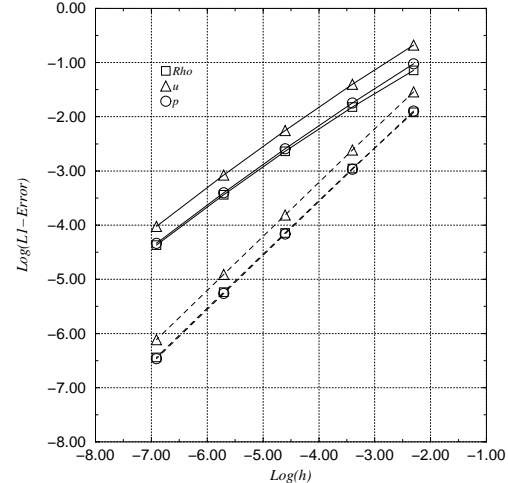

Figure 37: Relaxation d'énergie

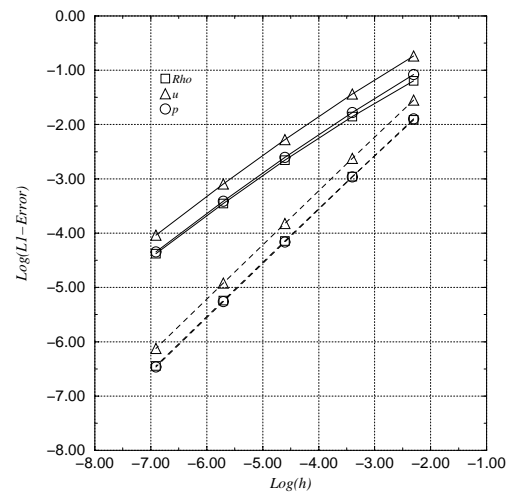

Figure 39: VFFC

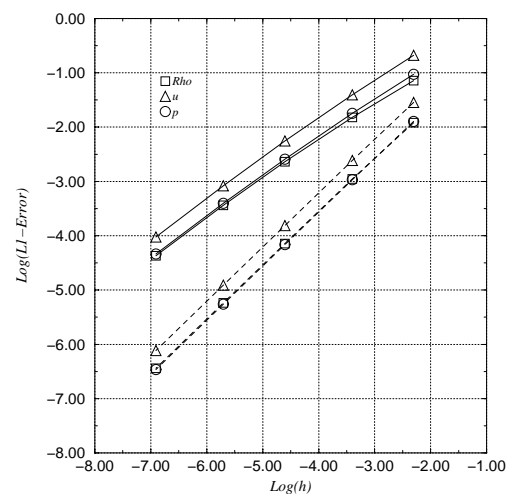

Figure 41: VFRoe ncv $(\rho, u, p)$

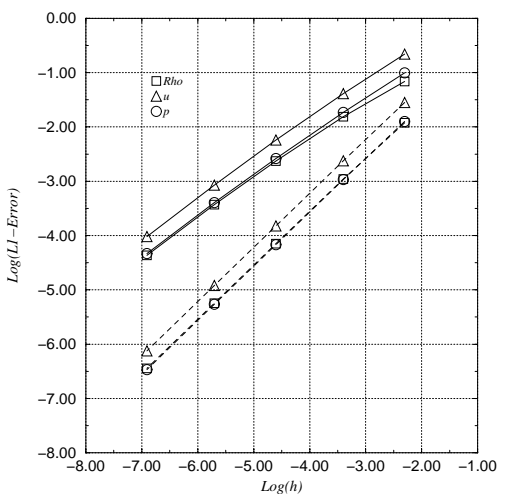

Figure 38: Rusanov

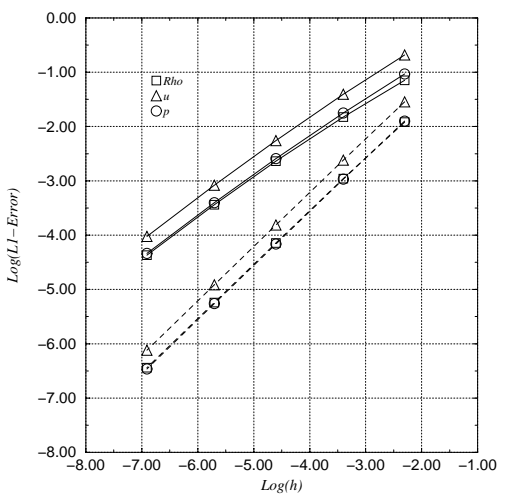

Figure 40: VFRoe

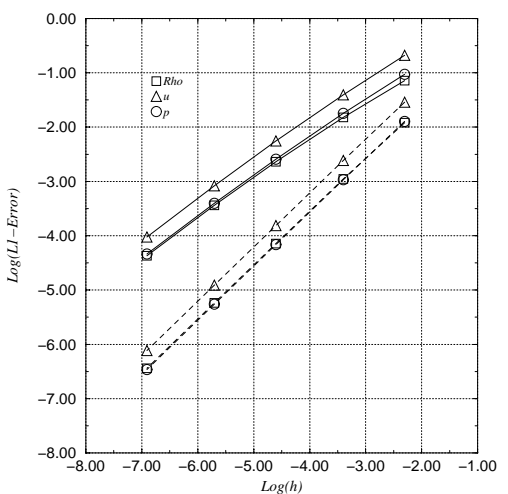

Figure 42: VFRoe ncv $(\tau, u, p)$ 
Perfect gas EOS - Symmetrical double shock wave

- Energy relaxation

\begin{tabular}{|c|c|c|}
\hline & 1st order & 2nd order \\
\hline$\rho$ & 1.062 & 0.935 \\
\hline$u$ & 1.157 & 1.156 \\
\hline$p$ & 1.050 & 1.017 \\
\hline
\end{tabular}

- Rusanov

\begin{tabular}{|c|c|c|}
\hline & 1st order & 2nd order \\
\hline$\rho$ & 1.060 & 1.028 \\
\hline$u$ & 1.056 & 1.115 \\
\hline$p$ & 0.996 & 1.001 \\
\hline
\end{tabular}

- VFFC

\begin{tabular}{|c|c|c|}
\hline & 1st order & 2nd order \\
\hline$\rho$ & 1.060 & 0.905 \\
\hline$u$ & 1.157 & 1.154 \\
\hline$p$ & 1.049 & 1.019 \\
\hline
\end{tabular}

- VFRoe

\begin{tabular}{|c|c|c|}
\hline & 1st order & 2nd order \\
\hline$\rho$ & 1.063 & 0.927 \\
\hline$u$ & 1.157 & 1.153 \\
\hline$p$ & 1.050 & 1.019 \\
\hline
\end{tabular}

- VFRoe ncv $(\rho, u, p)$

\begin{tabular}{|c|c|c|}
\hline & 1st order & 2nd order \\
\hline$\rho$ & 1.063 & 0.929 \\
\hline$u$ & 1.158 & 1.154 \\
\hline$p$ & 1.050 & 1.019 \\
\hline
\end{tabular}

- VFRoe ncv $(\tau, u, p)$

\begin{tabular}{|c|c|c|}
\hline & 1st order & 2nd order \\
\hline$\rho$ & 1.062 & 0.947 \\
\hline$u$ & 1.157 & 1.153 \\
\hline$p$ & 1.050 & 1.019 \\
\hline
\end{tabular}




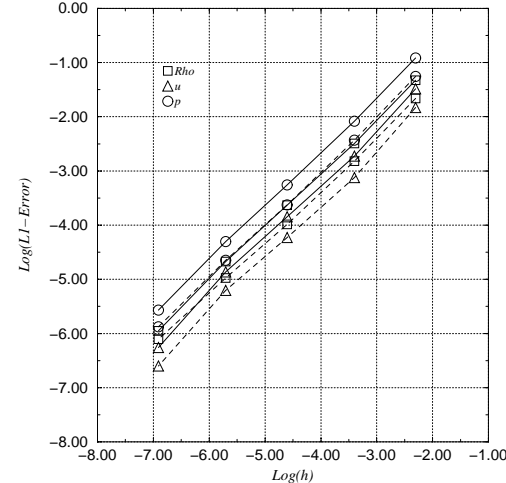

Figure 43: Relaxation d'énergie

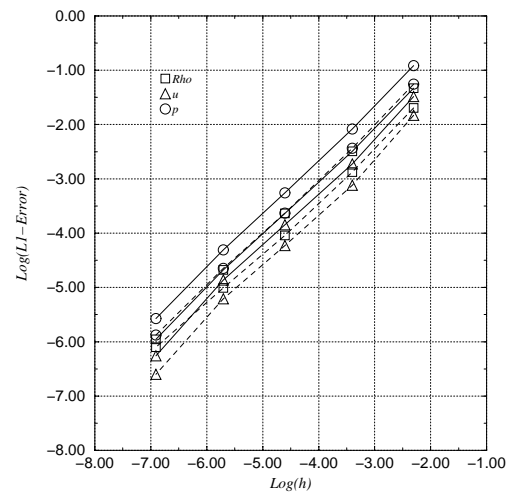

Figure 45: VFFC

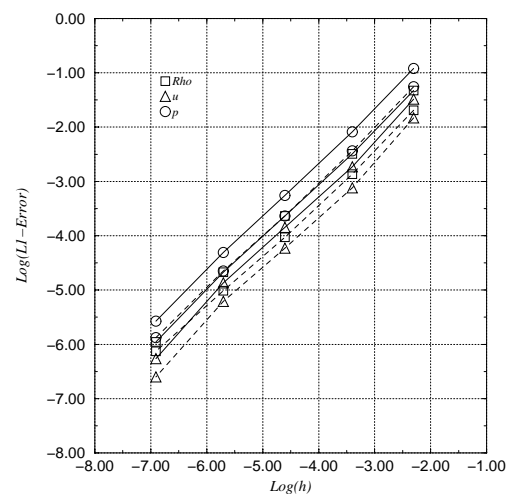

Figure 47: VFRoe ncv $(\rho, u, p)$

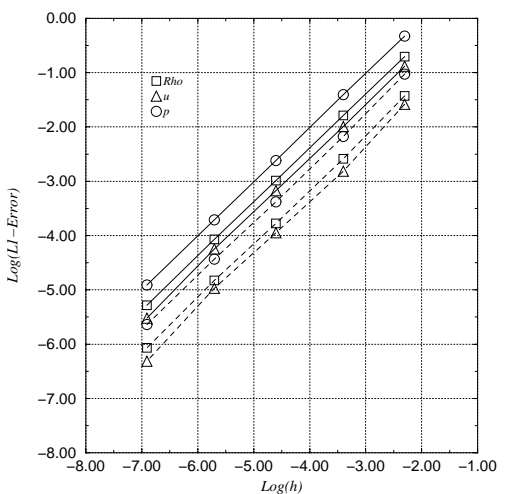

Figure 44: Rusanov

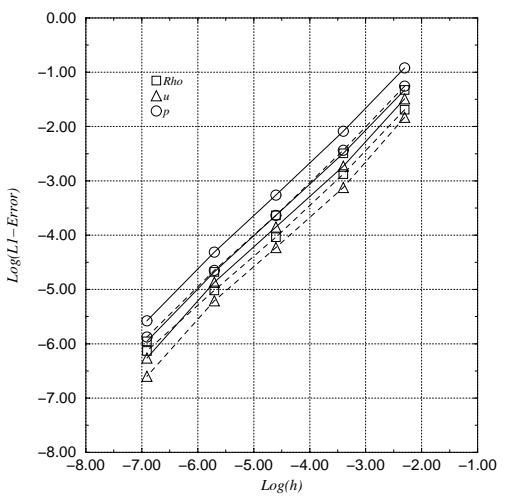

Figure 46: VFRoe

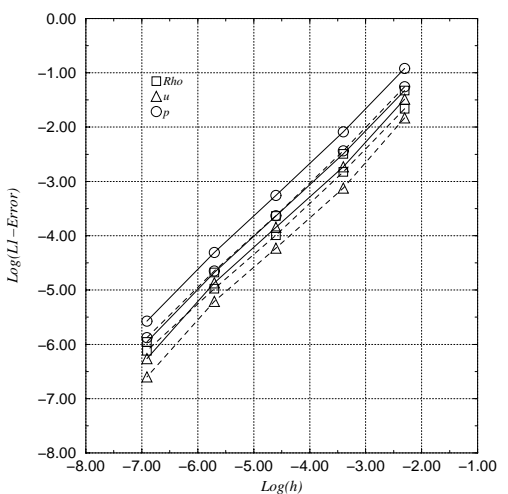

Figure 48: VFRoe ncv $(\tau, u, p)$ 


\section{Perfect gas EOS - Unsteady contact discontinuity}

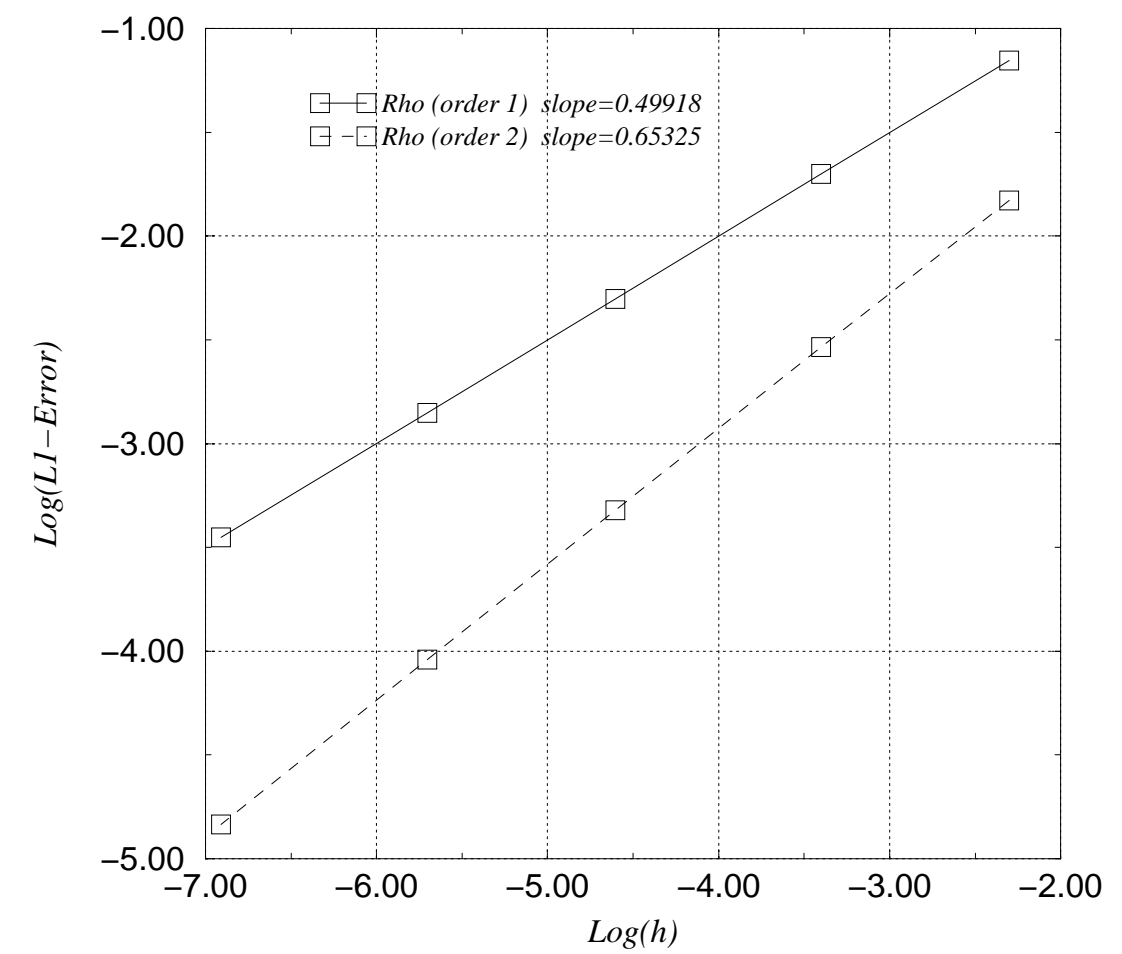

Figure 49: Case 2.1 : density

\section{Conclusion}

Several approximate Riemann solvers have been compared in this study. Some among them are based on an approximate Godunov scheme, applying various changes of variables in order to compute approximate values of state at the interface. These make use of conservative variable $W$, flux variable $F(W)$ or variable $(\rho, u, p)$ or $(\tau, u, p)$. The latter enables to preserve unsteady contact discontinuites provided the EOS agrees with some conditions (perfect gas EOS, Tammann EOS belong to the latter class). The practical or theorical behaviour of these schemes when computing steady shock wave, steady contact discontinuity, or vacuum has been investigated. All schemes perform rather well in all experiments, except in vacuum occurence or propagation over vacuum. One drawback of the VFFC scheme can be emphasized : when computing a double supersonic rarefaction wave (with or without vacuum occurence), this scheme blows up after a few time steps. Concerning VFRoe ncv $(\tau, u, p)$ and PVRS schemes, 
changing slightly the average state can increase their robustness and accuracy. The energy relaxation method applied with VFRoe ncv $(\tau, u, p)$ scheme has been computed too. The behaviour of this method is nearly the same as the original VFRoe ncv $(\tau, u, p)$ scheme. However, the energy relaxation method makes vanish non entropic shocks. The Rusanov scheme provides good results too, though it is slightly less accurate than other schemes investigated, due to important numerical diffusion. But the Rusanov scheme converges as fast as other schemes (in terms of mesh size exponent in the error norm). Moreover, it is the most robust scheme computed here, in particular in test cases with vacuum.

Moreover, a quantitative study has been presented. Solutions involving discontinuities have been investigated for first and second order shemes. Classical rates when restricting to smooth solutions are around 1 and 2 respectively. When the solution contains rarefaction waves or shock waves (without contact discontinuities), the rate becomes less than or equals to (for the second order schemes) 1. Restricting to a simple unsteady contact discontinuity, first order schemes provide a rate around $1 / 2$ and second order schemes provide a rate around $2 / 3$.

The framework of this paper has been restricted to the computation by Finite Volume schemes of a conservative and hyperbolic system, in one space dimension. Let us recall some extensions of methods used here, in different applications.

Of course, all schemes presented herein can be extended to $2 \mathrm{D}$ or $3 \mathrm{D}$ problems (see [4]). Rusanov (see [48]), Godunov (see [48]), VFFC (see [2]) and VFRoe ncv $(\tau, u, p)$ (see [9]) schemes have been applied to Euler equations with real gas EOS, Shallow Water equations (see [7]) and compressible gas-solid two phase flows (see [11]), with structured or unstructured meshes. Since these systems stay unchanged under frame rotation, a multidimensional framework may rely on a one dimension method (see [22]).

Some systems arising in CFD cannot be written under a conservative form, and thus, approximate jump relations must be proposed (see [14] and [10]). Some of the previous schemes have been extended to the non conservative formalism : Godunov (see [17]), Roe (see [3], [25], [26], [41]), VFRoe ncv (see [6], [8], [48]) and VFRoe (see [34], [1]).

Others non conservative systems are conditionnally hyperbolic, in particular focusing two fluid two phase flows (see [40]). Three main directions have been proposed up to now in the literature. The first consists in splitting the jacobian matrix in several matrices, which may be diagonalised in $\mathbb{R}$ (see [12]). The second way consists in using the sign of the real part of eigenvalues to choose the flux direction (see [36] and [2]). A third approach is based on a development in power series of eigenvalues and eigenvectors in terms of a small parameter (see [41], [46]). 


\section{A Numerical preservation of velocity and pres- sure through the contact discontinuity in Eu- ler equations}

We discuss in this appendix about schemes and state laws, in order to preserve velocity and pressure on the contact discontinuity, in a one dimension framework. We focus on initial conditions of a Riemann problem, with constant velocity and constant pressure. Schemes investigated here can be derived from the formalism of VFRoe ncv scheme, with variable:

$$
Y={ }^{t}(\varphi, u, p)
$$

where $\varphi=\varphi(\rho, s)$ ( $s$ denotes the specific entropy) must be independant of pressure $p$ (for instance $\varphi=\rho, \tau, \ldots$ ).

Restricting to regular solutions, Euler equations can be written related to $Y={ }^{t}(\varphi, u, p)$ as follows:

$$
Y_{, t}+A(Y) Y_{, x}=0
$$

where :

$$
A=\left(\begin{array}{ccc}
u & \rho \varphi, \rho & 0 \\
0 & u & \rho^{-1} \\
0 & \hat{\gamma} p & u
\end{array}\right)
$$

At each interface, we linearize the matrix $A(Y)$ to obtain a linear Riemann problem, which may be easily solved. Initial conditions are defined by the average values in cells apart from the considered interface $(i+1 / 2$ for instance)

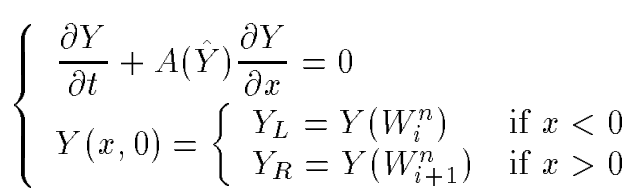

with $\hat{Y}$ such that $\hat{Y}(Y, Y)=Y$.

To compute the solution at the interface, we need to write the eigenstructure of the matrix $\mathrm{A}(\mathrm{Y})$. As usual, the eigenvalues are ( $c$ stands for the sound speed) :

$$
\lambda_{1}=u-c, \lambda_{2}=u, \lambda_{3}=u+c
$$

The associated right eigenvectors are :

$$
r_{1}(Y)=\left(\begin{array}{c}
\rho \varphi, \rho \\
-c \\
\rho c^{2}
\end{array}\right), r_{2}(Y)=\left(\begin{array}{l}
1 \\
0 \\
0
\end{array}\right), r_{3}(Y)=\left(\begin{array}{c}
\rho \varphi, \rho \\
c \\
\rho c^{2}
\end{array}\right)
$$

Left eigenvectors of $A(Y)$ are :

$$
l_{1}(Y)=\frac{1}{2 c^{2}}\left(\begin{array}{c}
0 \\
-c \\
\rho^{-1}
\end{array}\right), l_{2}(Y)=\frac{1}{c^{2}}\left(\begin{array}{c}
1 \\
0 \\
-\varphi_{, \rho}
\end{array}\right), l_{3}(Y)=\frac{1}{2 c^{2}}\left(\begin{array}{c}
0 \\
c \\
\rho^{-1}
\end{array}\right)
$$


In the following, we denote ${ }^{\sim}$ variables computed on the basis of $\bar{Y}$. The solution of the linear problem (10), when $x / t \neq \lambda_{k}, k=1,2,3$, is :

$$
\begin{aligned}
Y^{*}\left(x / t ; Y_{L}, Y_{R}\right) & =Y_{L}+\sum_{x / t>\widetilde{\lambda_{k}}}\left({ }^{t} \tilde{l_{k}} \cdot\left(Y_{R}-Y_{L}\right)\right) \tilde{r_{k}} \\
& =Y_{R}-\sum_{x / t<\widetilde{\lambda_{k}}}\left({ }^{t} \tilde{l_{k}} \cdot\left(Y_{R}-Y_{L}\right)\right) \widetilde{r_{k}}
\end{aligned}
$$

Since the three eigenvalues of the linear system are distinct, two intermediate states $Y_{1}$ and $Y_{2}$ may occur :

$$
\begin{aligned}
& Y_{1}=Y_{L}+\widetilde{\alpha_{1}} \widetilde{r_{1}} \\
& Y_{2}=Y_{R}-\widetilde{\alpha_{3}} \widetilde{r_{3}}
\end{aligned}
$$

with :

$$
\begin{aligned}
& \widetilde{\alpha_{1}}=-\frac{1}{2 \tilde{c}} \Delta u+\frac{1}{2 \tilde{\rho} \tilde{c}^{2}} \Delta p \\
& \widetilde{\alpha_{3}}=\frac{1}{2 \tilde{c}} \Delta u+\frac{1}{2 \tilde{\rho} \tilde{c}^{2}} \Delta p
\end{aligned}
$$

where $\Delta()=.(.)_{R}-(.)_{L}$. Note that the two intermediate states $Y_{1}$ and $Y_{2}$ do not depend on the choice of $\varphi$.

Recall that initial conditions investigated herein are unsteady contact discontinuity. Thus :

$$
\begin{aligned}
\Delta u=\Delta p=0 & \Rightarrow \widetilde{\alpha_{1}}=\widetilde{\alpha_{3}}=0 \\
& \Rightarrow Y_{1}=Y_{L} \text { and } Y_{2}=Y_{R}
\end{aligned}
$$

Note that these equalities are verified at each interface of the mesh. Hence, if we denote $\rho_{i+1 / 2}$ the numerical density of the problem (10) at the interface $i+1 / 2$, $u_{0}$ and $p_{0}$ initial velocity and pressure, the Finite Volume scheme applied to the mass conservation equation gives :

$$
\begin{aligned}
\rho_{i}^{n+1} & =\rho_{i}^{n}-\frac{\Delta t}{\Delta x}\left((\rho u)_{i+1 / 2}-(\rho u)_{i-1 / 2}\right) \\
& =\rho_{i}^{n}-\frac{\Delta t}{\Delta x} u_{0}\left(\rho_{i+1 / 2}-\rho_{i-1 / 2}\right)
\end{aligned}
$$

Now, if we apply the Finite Volume scheme to the momentum conservation equation, it gives:

$$
\begin{aligned}
(\rho u)_{i}^{n+1} & =(\rho u)_{i}^{n}-\frac{\Delta t}{\Delta x}\left(\left(\rho u^{2}+p\right)_{i+1 / 2}-\left(\rho u^{2}+p\right)_{i-1 / 2}\right) \\
& =(\rho u)_{i}^{n}-\frac{\Delta t}{\Delta x}\left(\left(\rho_{i+1 / 2} u_{0}^{2}+p_{0}\right)-\left(\rho_{i-1 / 2} u_{0}^{2}+p_{0}\right)\right) \\
& =(\rho u)_{i}^{n}-\frac{\Delta t}{\Delta x} u_{0}^{2}\left(\rho_{i+1 / 2}-\rho_{i-1 / 2}\right) \\
& =u_{0}\left(\rho_{i}^{n}-\frac{\Delta t}{\Delta x} u_{0}\left(\rho_{i+1 / 2}-\rho_{i-1 / 2}\right)\right) \\
& =u_{0} \rho_{i}^{n+1}
\end{aligned}
$$


Thus, we have $u_{i}^{n+1}=u_{0}, \forall i \in \mathbb{Z}$.

To study the discrete preservation of pressure, let us write the Finite Volume scheme applied to energy conservation equation :

$$
\begin{aligned}
E_{i}^{n+1} & =E_{i}^{n}-\frac{\Delta t}{\Delta x}\left((u(E+p))_{i+1 / 2}-(u(E+p))_{i-1 / 2}\right) \\
& =E_{i}^{n}-\frac{\Delta t}{\Delta x} u_{0}\left(E_{i+1 / 2}-E_{i-1 / 2}\right)
\end{aligned}
$$

Energy is defined by $E=\rho \varepsilon+\frac{1}{2} \rho u^{2}$. Thus, we have:

$$
(\rho \varepsilon)_{i}^{n+1}=(\rho \varepsilon)_{i}^{n}-\frac{\Delta t}{\Delta x} u_{0}\left((\rho \varepsilon)_{i+1 / 2}-(\rho \varepsilon)_{i-1 / 2}\right)
$$

Let us assume that the equation of state can be written under the form :

$$
\rho \varepsilon=f(p)+b \rho+c
$$

where $b$ and $c$ are real constants, and $f$ a inversible function (for instance perfect gas EOS, Tammann EOS, ...). If we introduce this equation of state in the previous equation, it gives :

$$
\begin{aligned}
(f(p)+b \rho+c)_{i}^{n+1}= & (f(p)+b \rho+c)_{i}^{n} \\
& -\frac{\Delta t}{\Delta x} u_{0}\left((f(p)+b \rho+c)_{i+1 / 2}-(f(p)+b \rho+c)_{i-1 / 2}\right) \\
f\left(p_{i}^{n+1}\right)+b \rho_{i}^{n+1}+c= & f\left(p_{0}\right)+b \rho_{i}^{n}+c \\
& -\frac{\Delta t}{\Delta x} u_{0}\left(\left(f\left(p_{0}\right)-f\left(p_{0}\right)\right)+b\left(\rho_{i+1 / 2}-\rho_{i-1 / 2}\right)+(c-c)\right) \\
f\left(p_{i}^{n+1}\right)= & f\left(p_{0}\right)
\end{aligned}
$$

Thus, $p_{i}^{n+1}=p_{0}$.

Hence, if a state law can be written under the form (11), then a VFRoe ncv scheme, whose variable is $(\varphi, u, p)$-like, maintains velocity and pressure constant.

Moreover, if the contact discontinuity is steady (ie $u_{0}=0$ ), we can remark that the VFRoe ncv $(\varphi, u, p)$ scheme preserves pressure and velocity exactly constant, whatever the state law considered.

\section{References}

[1] C. Berthon, F. Coquel, J.M. Hérard and M. Uhlmann, An approximate solution of the Riemann problem for a realizable second moment turbulent closure, to appear in Shock Waves.

[2] M. BoucKer, Modélisation numérique multidimensionnelle d'écoulements diphasiques liquide-gaz en régimes transitoire et permanent: méthodes et applications, PhD thesis, École Normale Supérieure de Cachan, France, December 1998. 
[3] G. Brun, J.M. Hérard, D. Jeandel and M. Uhlmann, An approximate Roe-type Riemann solver for a class of realizable second order closures, Int. J. of Comp. Fluid Dyn., 2000, vol. 13-3, pp. 233-249.

[4] T. Buffard, Analyse de quelques méthodes de volumes finis non structurés pour la résolution des équations d'Euler, $\mathrm{PhD}$ thesis, Université Paris VI, France, 1993.

[5] T. Buffard, T. Gallouët and J.M. HÉRaRd, Schéma VFRoe en variables caractéristiques. Principe de base et applications aux gaz réels, EDFDER Report HE-41/96/041/A, 1996. In French.

[6] - A naive Godunov scheme to compute a non-conservative hyperbolic system, Int. Series Num. Math., 1998, vol. 129, pp. 129-138.

[7] — A naive Godunov scheme to solve shallow-water equations, C. $R$. Acad. Sci. Paris, 1998, vol. I-326, pp. 885-890.

[8] — An approximate Godunov scheme to compute turbulent real gas flow models, AIA A paper 99-3349, 1999.

[9] - A sequel to a rough Godunov scheme. Application to real gas flows, Computers and Fluids, 2000, vol. 29-7, pp. 813-847.

[10] J.F. Colombeau, Multiplication of distributions, Springer Verlag, 1992.

[11] L. Combe AND J.M. HÉRARD, Principe du maximum pour un modèle diphasique gaz-solide à trois équations, EDF-DER Report HE-41/96/045/A, 1996. In French.

[12] - Finite volume algorithm to compute dense compressible gas-solid flows, AIA A journal, 1999, vol. 37, pp. 337-345.

[13] F. Coquel and B. Perthame, Relaxation of energy and approximate Riemann solvers for general pressure laws in fluid dynamics equations, SIAM J. Numer. Anal., 1998, vol. 35-6, pp. 2223-2249. In Memory of Ami Harten.

[14] G. Dal Maso, P.G. Le Floch and F. Murat, Definition and weak stability of non conservative products, J. Math. Pures Appl., 1995, vol. 74, pp. $483-548$.

[15] B. Einfeldt, C.D. Munz, P.L. Roe and B. Suögreen, On Godunovtype methods near low densities, J. Comp. Phys., 1991, vol. 92-2, pp. 273295 .

[16] R. Eymard, T. Gallouët and R. Herbin, Finite Volume Methods, In Handbook of Numerical Analysis (Vol. VII), editors : P.G. Ciarlet and J.L. Lions, pp. 729-1020, North-Holland, pp. 729-1020, 2000. 
[17] A. Forestier, J.M. Hérard and X. Louis, A Godunov type solver to compute turbulent compressible flows, C. R. Acad. Sci. Paris, 1997, vol. I-324, pp. 919-926.

[18] T. Gallouët, J.M. Hérard and N. Seguin, An hybrid scheme to compute contact discontinuities in Euler systems, EDF-DRD Report HI81/01/011/A, Submitted for publication, 2001.

[19] T. Galloü̈t and J.M. Masella, A rough Godunov scheme, $C$. R. Acad. Sci. Paris, 1996, vol. I-323, pp. 77-84.

[20] B. Gest, J.M. HÉraRd And L. Kloetzer, Validation d'un algorithme implicite volumes finis pour gaz réels, EDF-DER Report HE-41/98/041/A, 1998. In French.

[21] J.M. Ghidaglia, A. Kumbaro and G. Le Coq, Une approche volume fini à flux caractéristiques pour la résolution numérique des systèmes hyperboliques de lois de conservation, C. R. Acad. Sci. Paris, 1996, vol. I-322, pp. $981-988$.

[22] E. Godlewski and P.A. Raviart, Numerical approximation of hyperbolic systems of conservation laws, Springer Verlag, 1996.

[23] S.K. Godunov, A difference method for numerical calculation of discontinuous equations of hydrodynamics, Mat. Sb., 1959, pp. 271-300. In Russian.

[24] A. HaRTen AND J.M. Hyman, A self-adjusting grid for the computation of weak solutions of hyperbolic conservation laws, J. Comp. Phys., 1983, vol. 50, pp. 235-269.

[25] J.M. HÉRARD, Solveur de Riemann approché pour un système hyperbolique non conservatif issu de la turbulence compressible, EDF-DER Report HE41/95/009/A, 1995. In French.

[26] J.M. HÉrard, A. Forestier and X. Louis, A non strictly hyperbolic system to describe compressible turbulence, EDF-DER Report HE41/94/011/A, 1994.

[27] A. In, Numerical evaluation of an energy relaxation method for inviscid real fluids, to appear in SIAM J. Sci. Comp.

[28] — Méthodes numériques pour les équations de la dynamique des gaz complexes et écoulements diphasiques, PhD thesis, Université Paris VI, France, October 1999.

[29] M..J. Ivings, D.M. Causon and E.F. Toro, On Riemann solvers for compressible flows, Int. J. Num. Meth. Fluids, 1998, vol. 28, pp. 395-418.

[30] R. KeE, J. Miller AND T. JefFERson, Chemkin: a general purpose, problem independant transportable fortran chemical kinetics code package, SAND Report 80-8003, Sandia National Laboratories. 
[31] A. Kumbaro, Schéma volume fini à flux caractéristiques - implémentation, tests et résultats numériques, EDF-DER Report HT-30/95/017/A, 1995. In French.

[32] A. Letellier and A. Forestier, Le problème de Riemann en fluide quelconque, CEA-DMT Report 93/451, 1993. In French.

[33] R.J. LeVEque, Wave propagation algorithms for multidimensional hyperbolic systems, J. Comp. Phys., 1997, vol. 131, pp. 327-353.

[34] J.M. Maselda, Quelques méthodes numériques pour les écoulements diphasiques bi-fluides en conduites pétrolières, $\mathrm{PhD}$ thesis, Université Paris VI, France, May 1997.

[35] J.M. Masella, I. Faille and T. Gallouët, On an approximate Godunov scheme, Int. J. of Comp. Fluid Dyn., 1999, vol. 12, pp. 133-149.

[36] S. Mimouni, M. Boucker, G. Le Cog, J.M. Ghidaglia and al., An overview of the VFFC-methods and tools for the simulation of two-phase flows, EDF-DER Report HT-33/99/006/A, 1999.

[37] P. Rascle and O. Morvant, Interface utilisateur de Thetis - THErmodynamique en Tables d'InterpolationS, EDF-DER Report HT-13/95021B, 1995. In French.

[38] P.L. Roe, Approximate Riemann solvers, parameter vectors and difference schemes, J. Comp. Phys., 1981, vol. 43, pp. 357-372.

[39] V.V. Rusanov, Calculation of interaction of non-steady shock waves with obstacles, J. Comp. Math. Phys. USSR, 1961, vol. 1, pp. 267-279.

[40] L. Sainsaulieu, Contribution à la modélisation mathématique et numérique des écoulements diphasiques constitués d'un nuage de particules dans un écoulement de gaz, Thèse d'habilitation, Université Paris VI, France, 1995 .

[41] _ Finite volume approximations of two phase-fluid flows based on approximate Roe-type Riemann solver, J. Comp. Phys., 1995, vol. 121, pp. 128.

[42] R. Saurel and R. Abgrall, A simple method for compressible multifluid flows, SIAM J. Sci. Comp., 1999, vol. 21-3, pp. 1115-1145.

[43] N. Seguin, Comparaison numérique de schémas Volumes Finis pour les équations d'Euler en gaz parfaits et réels, EDF-DRD Report HI81/00/010/A, 2000, pp. 1-451. In French.

[44] E.F. Toro, A linearised Riemann solver for time-dependent Euler equations of gas dynamics, Proc. R. Soc. Lond. A., 1991, vol. 434, pp. 683-693. 
[45] - Riemann solvers and numerical methods for fluid dynamics, Springer Verlag, 1997.

[46] I. Toumi and A. Kumbaro, An approximate linearized Riemann solver of a two-fluid model, J. Comp. Phys., 1996, vol. 124, pp. 286-300.

[47] B. Van Leer, Toward the ultimate conservative difference scheme V. A second order sequel to Godunov's method, J. Comp. Phys., 1979, vol. 32, p. 101.

[48] E. XEuXet, Comparaison de solveurs numériques pour le traitement de la turbulence bifluide, PhD thesis, Université d'Evry, France, June 1999. 